Homology, Homotopy and Applications, vol.11(2), 2009, pp.75-132

\title{
THE HUNTING OF THE HOPF RING
}

\author{
ANDREW STACEY AND SARAH WHITEHOUSE
}

(communicated by Ulf Rehmann)

\begin{abstract}
We provide a new algebraic description of the structure on the set of all unstable cohomology operations for a suitable generalised cohomology theory, $E^{*}(-)$. Our description is as a graded and completed version of a Tall-Wraith monoid. The $E^{*}$-cohomology of a space $X$ is a module for this Tall-Wraith monoid. We also show that the corresponding Hopf ring of unstable co-operations is a module for the Tall-Wraith monoid of unstable operations. Further examples are provided by considering operations from one theory to another.
\end{abstract}

\section{Introduction}

In this paper we provide a new algebraic description of the structure on the set of all unstable cohomology operations for a suitable generalised cohomology theory, say $E^{*}(-)$. The bigraded set of unstable operations for $E^{*}(-)$ is identified by the usual Yoneda lemma argument with the bigraded set of cohomology groups of the representing spaces, $\left\{E^{k}\left(\underline{E}_{l}\right)\right\}$. This has considerable structure and it is natural to ask how to best describe it.

To date, the most comprehensive work in this area is due to Boardman, Johnson, and Wilson [BJW95]. They provide the following descriptions of this structure.

1. The unstable operations of a suitable cohomology theory define a monad on the category of complete, Hausdorff, filtered, graded, commutative, unital $E^{*}$ algebras.

2. The unstable operations of a suitable cohomology theory are dual to the enriched Hopf ring of the corresponding co-operations.

The Hopf ring part-i.e., ignoring the enrichment - of the second answer is, of course, a well-established and important notion in algebraic topology. Since the work of Ravenel and Wilson [RW77], the language of Hopf rings has been widely used and the structures of the Hopf rings associated to many important cohomology theories have been calculated; see, for example, [CMS02, RW77, RW96, Wil84]. A useful introduction to Hopf rings, with further references, can be found in [Wil00].

The authors acknowledge the support of the EPSRC, grant no.: GR/S76823/01.

Received June 13, 2008; published on August 23, 2009.

2000 Mathematics Subject Classification: Primary: 55S25; Secondary: 55N20, 16W99.

Key words and phrases: unstable cohomology operations, Hopf ring.

This article is available at http://intlpress.com/HHA/v11/n2/a6

Copyright (C) 2009, Andrew Stacey and Sarah Whitehouse. Permission to copy for private use granted. 
However in considering a Hopf ring, we have not yet taken into account one of the most obvious pieces of structure: that operations may be composed. A Hopf ring does not include any structure which dualises to composition of operations. The enrichment in the second answer encodes the dual of composition. The enriched Hopf ring structures of several important cohomology theories are described in [BJW95].

The first answer, describing operations as a monad on a suitable category, certainly includes the composition as a fundamental part of the structure. But this answer does not explicitly describe the internal structure on the set of operations; instead it specifies the action of operations on some category. In particular, this approach does not lend itself to specifying the structure of operations via generators and relations.

Of these descriptions, that of an unenriched Hopf ring has proved to be more amenable to further study than either that of an enriched Hopf ring or that of a monad on a suitable category of algebras. Indeed, whilst there have been many papers written using the theory of Hopf rings there have been very few using either enriched Hopf rings or the monadic description. Thus one of the most fundamental properties of operations - that they can be composed - has been largely ignored.

Our new description is algebraic in nature and we employ the language of general or universal algebra to express it. A key advantage of this approach is that it describes the structure of unstable operations - including composition - directly. Another important advantage is that it allows for descriptions of unstable operations via generators and relations. Such descriptions are expected to shed light on the relationships between stable, additive, and unstable operations. For the Morava Ktheories and related cohomology theories, these relationships were studied in [SW08]; the results of this paper provide the foundations for a very explicit description, via generators and relations, of the splitting of stable operations from unstable operations given in [SW08]. We expect to develop this point of view for familiar examples in future work.

The relationships between our description and those of Boardman, Johnson, and Wilson are simple to describe. From our algebraic description one can define the corresponding monad and recover its action on the category of suitable algebras. The enrichment part of an enriched Hopf ring now appears as a natural action of the set of operations on the set of co-operations; in particular, all of the identities that appear in the enrichment are encoded in this action.

Roughly speaking, our answer is that the unstable operations of a suitable theory have the structure of a graded, completed Tall-Wraith monoid; a term that we shall now explain.

Let $\mathcal{V}$ be a variety of algebras, in the sense of general or universal algebra. A TallWraith $\mathcal{V}$-monoid is a set with precisely the algebraic structure required for it to act on $\mathcal{V}$-algebras. To make this precise, one considers the category of co- $\mathcal{V}$-algebra objects in $\mathcal{V}$. This is equivalent to the category of representable functors from $\mathcal{V}$ to $\mathcal{V}$ and so it has a monoidal structure corresponding to composition of functors. A Tall-Wraith $\mathcal{V}$ monoid is then defined to be a monoid in this category. One example is very familiar: a ring is a Tall-Wraith $\mathcal{V}$-monoid for $\mathcal{V}$ the category of abelian groups. The case originally considered by Tall and Wraith in [TW70], under the name biring triple, was for $\mathcal{V}$ the category of commutative unital rings. Recently, Borger and Weiland [BW05] rediscovered this and extended it to the case where $\mathcal{V}$ is the category of 
commutative unital $k$-algebras, for a commutative unital ring $k$. They adopted the term plethory in that situation; thus a plethory is that-which-acts-on-algebras. This is clearly relevant to our purposes as unstable cohomology operations of multiplicative cohomology theories act on the cohomology algebras.

It is also clear that there remains work to be done, because the cohomology theories that we are considering are graded and topologised. The grading introduces no technical difficulties: varieties of graded algebras have been studied for almost as long as varieties of ordinary algebras; they are also known as many-sorted algebras in the literature. We arrive at Tall-Wraith $\mathcal{V}^{*}$-monoids for $\mathcal{V}^{*}$ a variety of graded algebras. These are naturally bi-graded, as are unstable cohomology operations.

The main work of this paper comes in the extension of Tall-Wraith monoids to a suitably topologised context. For $E^{*}(-)$ a multiplicative cohomology theory and $X$ a $C W$-complex, $E^{*}(X)$ is given the pro-finite topology; that is, the filtration topology for the filtration by the ideals $\left\{\operatorname{ker} i^{*}: E^{*}(X) \rightarrow E^{*}\left(X_{f}\right)\right\}$ for all inclusions of finite subcomplexes $i: X_{f} \rightarrow X$. We therefore develop a theory of filtered Tall-Wraith monoids so that our description of unstable operations takes into account the profinite filtrations. While there are various notions of filtered objects in a (suitable) category in the literature, we have not been able to find a set-up suited to our needs. Therefore we introduce a suitable definition of filtered objects in a category to model the topology; in this setting we introduce iso-filtrations as the generalisation of complete, Hausdorff spaces. We denote the resulting category of iso-filtered objects by $\overrightarrow{\mathcal{V}^{*}}$.

The main work of this paper is here. The set of unstable operations in a suitable cohomology theory is a co- $\mathcal{V}^{*}$-algebra object in $\overrightarrow{\mathcal{V}^{*}}$. Now the monoidal structure in the unfiltered cases comes from the fact that a co- $\mathcal{V}$-algebra object in $\mathcal{V}$ represents a functor from $\mathcal{V}$ to $\mathcal{V}$ and so two such can be composed. However, a co- $\mathcal{V}^{*}$-algebra object in $\overrightarrow{\mathcal{V}^{*}}$ represents a functor from $\overrightarrow{\mathcal{V}^{*}}$ to $\mathcal{V}^{*}$ and so two such cannot be composed in an obvious way. What we show is that a functor $\overrightarrow{\mathcal{V}^{*}} \rightarrow \mathcal{V}^{*}$ has a natural lift to a functor $\overrightarrow{\mathcal{V}^{*}} \rightarrow \overrightarrow{\mathcal{V}^{*}}$ whereupon we can consider compositions. To show that this induces a monoidal structure on the category of co- $\mathcal{V}^{*}$-algebra objects in $\overrightarrow{\mathcal{V}^{*}}$ we need to show that this lift "plays well" with adjoints. This is not trivial and is the main technical result of this paper.

This allows us to formulate the notion of a Tall-Wraith $\overrightarrow{\mathcal{V}^{*}}$-monoid.

Once we have established the general theory of graded filtered Tall-Wraith monoids, the applications to suitable generalised cohomology theories are straightforward. We can now state precisely our description of the structure on the set of all unstable operations. We adopt standard notation related to a cohomology theory $E^{*}(-)$, so the representing spaces are denoted by $\underline{E}_{k}($ for $k \in \mathbb{Z})$, the corresponding homology theory is denoted by $E_{*}(-)$, and the coefficient ring by $E^{*}$.

Theorem A. Let $E^{*}(-)$ be a graded, commutative, multiplicative, cohomology theory. Let $\mathcal{V}^{*}$ be the variety of graded, commutative, unital $E^{*}$-algebras. Suppose that $E_{*}\left(\underline{E}_{k}\right)$ is free as an $E^{*}$-module for each $k$. Then $E^{*}\left(\underline{E}_{*}\right)$ is a Tall-Wraith $\overrightarrow{\mathcal{V}^{*}}$-monoid.

As noted in [BJW95], the freeness hypothesis of Theorem A is satisfied for $H \mathbb{F}_{p}$, $B P, M U, K(n)$, and $K U$. 
There is a natural notion of a module for a Tall-Wraith monoid. The cohomology of spaces provides examples of modules for the Tall-Wraith monoid of unstable operations.

Theorem B. Let $E^{*}(-)$ and $\mathcal{V}^{*}$ be as in Theorem A. Let $X$ be a topological space. The natural morphisms of sets

$$
E^{k}(X) \rightarrow \mathcal{V}^{*}\left(E^{*}\left(\underline{E}_{k}\right), E^{*}(X)\right)
$$

make the completed cohomology of $X, \overline{E^{*}}(X)$, into a module for the Tall-Wraith $\overrightarrow{\mathcal{V}^{*}}$-monoid of unstable operations $E^{*}\left(\underline{E}_{*}\right)$.

As mentioned earlier, the Hopf ring of co-operations is also a module for the TallWraith monoid of operations, and it is this extra structure that is encoded in the term enriched. Further examples come from considering operations and co-operations from one theory to another.

Theorem C. Let $E^{*}(-)$ and $\mathcal{V}^{*}$ be as in Theorem A. Let $F^{*}(-)$ be another graded, commutative, multiplicative cohomology theory. Suppose that each $F_{*}\left(\underline{E}_{k}\right)$ is free as an $F^{*}$-module. Then the following statements are true.

1. The bigraded set $F^{*}\left(\underline{E}_{*}\right)$ of unstable operations $E^{*}(-) \rightarrow F^{*}(-)$ has the structure of a module for the Tall-Wraith $\overrightarrow{\mathcal{V}^{*}}$-monoid $E^{*}\left(\underline{E}_{*}\right)$.

2. The Hopf ring $F_{*}\left(\underline{E}_{*}\right)$ is a module for $E^{*}\left(\underline{E}_{*}\right)$.

3. Let $X$ be a space such that $F_{*}(X)$ is free as an $F^{*}$-module. Let $\mathcal{C}_{F^{*}}$ be the category of $F^{*}$-co-algebras. Then the natural morphisms of sets

$$
E^{k}(X) \rightarrow \mathcal{C}_{F^{*}}\left(F_{*}(X), F_{*}\left(\underline{E}_{k}\right)\right)
$$

extend to a morphism of modules for $E^{*}\left(\underline{E}_{*}\right)$.

This paper is organised as follows. Section 2 covers background material from general algebra, in both the ungraded and graded contexts. The main aim of this section is to establish the necessary conditions to consider Tall-Wraith monoids and certain important related concepts. Section 3 is concerned with setting out the necessary details of the theory of filtered objects in a category. In this section we consider several types of filtrations and the relationships between them. As we shall want to apply the constructions of general algebra in such categories we are also concerned with establishing the categorical properties of these categories of filtered objects. The main technical work of this paper is in this section and concerns functors between categories of filtered objects. Section 4 brings together the work of the preceding sections by considering Tall-Wraith monoids in the filtered context. Section 5 applies the results to the examples arising in algebraic topology from suitable generalised cohomology theories, thus proving the theorems stated above.

The reader more interested in the results than the method by which they are demonstrated may prefer to read this paper in reverse.

Finally, we wish to acknowledge the work of Boardman, Johnson, and Wilson in understanding unstable operations. Although this paper does not depend on [BJW95] mathematically, it was an invaluable resource as a guide to determine the form of our final answer. 


\section{General Algebra}

In this section we shall expand a little on the basic constructions of general algebra. The results quoted in this section are all standard results from that field. For ungraded algebra objects in Set, these results can be found in any good introduction to the subject, for example in [Ber98]. The more general cases can be found in the wider literature, for example in [KPMS82]. We record these results here without proofs to establish notation and as a quick reference for the rest of the paper. For those initiates of the deeper secrets of general algebra we mention that we are only considering algebras of finite arity and so we can assume that our identities are specified by finite sets.

We start by summarising the results that we need in the arena of ungraded algebras, also known as single-sorted or homogeneous algebras. We shall then explain how this generalises to graded algebras, also known as many-sorted or heterogeneous algebras.

\subsection{Ungraded Algebra}

Definition 2.1. Let $\mathcal{D}$ be a category with finite products.

1. A type $\Omega$ is a pair $(|\Omega|, n)$ where $|\Omega|$ is a set and $n:|\Omega| \rightarrow \mathbb{N}_{0}$ is a morphism of sets called the arity morphism.

2. An $\Omega$-algebra object, $H$, in $\mathcal{D}$ consists of an object in $\mathcal{D},|H|$, together with, for each $\omega \in|\Omega|$, a $\mathcal{D}$-morphism $\omega_{H}:|H|^{n(\omega)} \rightarrow|H|$; these morphisms are called the operations of the $\Omega$-algebra object in $\mathcal{D}$. A morphism of $\Omega$-algebra objects in $\mathcal{D}$ is a morphism of the underlying objects in $\mathcal{D}$ which intertwines the operations.

3. An $\Omega$-algebra is an $\Omega$-algebra object in Set.

4. We denote the category of $\Omega$-algebra objects in $\mathcal{D}$ by $\mathcal{D} \Omega$ and the category of $\Omega$-algebra objects in Set by $\Omega$. We refer to the functor $\mathcal{D} \Omega \rightarrow \mathcal{D}$ which assigns to an $\Omega$-algebra object in $\mathcal{D}$ the underlying object in $\mathcal{D}$ as the forgetful functor. We write the underlying object in $\mathcal{D}$ of an $\Omega$-algebra object, $H$, in $\mathcal{D}$ as $|H|$.

We trust to context to distinguish between $\Omega$ the type and $\Omega$ the resulting category of algebras.

One of the key initial results in general algebra is the following characterisation of $\Omega$-algebra object structures.

Proposition 2.2. To give an object, $|H|$, in $\mathcal{D}$ the structure of an $\Omega$-algebra object is equivalent to giving a lift of the contravariant hom-functor $\mathcal{D}(-,|H|): \mathcal{D} \rightarrow$ Set to a functor $\mathcal{D} \rightarrow \Omega$.

If $H$ is an $\Omega$-algebra object in $\mathcal{D}$ and $D$ is an object in $\mathcal{D}$ we shall write

$$
\mathcal{D}(D, H)
$$

for the corresponding $\Omega$-algebra with underlying set $\mathcal{D}(D,|H|)$. The operations on $\mathcal{D}(D, H)$ are straightforward; let $\omega \in|\Omega|$ be an element of arity $n$ and let $\omega_{H}:|H|^{n} \rightarrow$ $|H|$ be the operation on $H$. Then the corresponding operation on $\mathcal{D}(D, H)$ sends the 
$\mathcal{D}$-morphisms $f_{1}, \ldots, f_{n}: D \rightarrow|H|$ to the $\mathcal{D}$-morphism

$$
D \stackrel{\Delta^{n}}{\longrightarrow} D^{n} \stackrel{f_{1} \times \cdots \times f_{n}}{\longrightarrow}|H|^{n} \stackrel{\omega_{H}}{\longrightarrow}|H|
$$

where $\Delta^{n}: D \rightarrow D^{n}$ is the $n$-fold diagonal morphism.

$\Omega$-algebras and $\Omega$-algebra objects simply have operations, they are not constrained to satisfy any particular identities. To consider identities, we need to know about free $\Omega$-algebras.

Proposition 2.3. Let $\mathcal{D}$ be a category with finite products which is closed under filtered co-limits and such that finite products commute with filtered co-limits. Then the forgetful functor $\mathcal{D} \Omega \rightarrow \mathcal{D}$ has a left adjoint, $\mathfrak{F}_{\Omega}: \mathcal{D} \rightarrow \mathcal{D} \Omega$, which is called the free $\Omega$-algebra functor.

For identities, we only need to know about free $\Omega$-algebras in Set.

Definition 2.4. Let $\Omega$ be a type. An identity for $\Omega$-algebras is a triple $(X, u, v)$ where $X$ is a (finite) set and $u, v \in\left|\mathfrak{F}_{\Omega}(X)\right|$.

Let $H$ be an $\Omega$-algebra object in $\mathcal{D}$. An identity for $\Omega$-algebras, $(X, u, v)$, induces natural $\mathcal{D}$-morphisms

$$
u_{H}, v_{H}:|H|^{X} \rightarrow|H| .
$$

These are defined as follows.

The canonical projections $|H|^{X} \rightarrow|H|$ in $\mathcal{D}$ define a set morphism

$$
X \rightarrow \mathcal{D}\left(|H|^{X},|H|\right) .
$$

As $H$ is an $\Omega$-algebra object in $\mathcal{D}$, the right hand side of this is the underlying set of an $\Omega$-algebra. Using the adjunction we therefore have a morphism of $\Omega$-algebras

$$
\mathfrak{F}_{\Omega}(X) \rightarrow \mathcal{D}\left(|H|^{X}, H\right)
$$

and thus the elements $u, v \in\left|\mathfrak{F}_{\Omega}(X)\right|$ define elements in the underlying set of the $\Omega$ algebra $\mathcal{D}\left(|H|^{X}, H\right)$ which is $\mathcal{D}\left(|H|^{X},|H|\right)$. Thus we have the required $\mathcal{D}$-morphisms $u_{H}, v_{H}:|H|^{X} \rightarrow|H|$.

Definition 2.5. An $\Omega$-algebra object, $H$, in $\mathcal{D}$ is said to satisfy the identity $(X, u, v)$ if the two induced $\mathcal{D}$-morphisms $u_{H}, v_{H}:|H|^{X} \rightarrow|H|$ are the same.

Definition 2.6. A variety of algebras, $\mathcal{V}$, is specified by a type, $\Omega$, and a set of identities for $\Omega$-algebras, $J$. It is the full subcategory of $\Omega$ consisting of all $\Omega$-algebras which satisfy all of the identities in $J$.

The pair $(\Omega, J)$ is a presentation of $\mathcal{V}$.

Let $\mathcal{D}$ be a category with finite products, $\mathcal{V}$ a variety of algebras with presentation $(\Omega, J)$. The category of $\mathcal{V}$-algebra objects in $\mathcal{D}, \mathcal{D} \mathcal{V}$, is the full subcategory of $\mathcal{D} \Omega$ consisting of all $\Omega$-algebra objects in $\mathcal{D}$ which satisfy all of the identities in $J$.

As is well-known, presentations are not unique.

Proposition 2.2 holds in the presence of identities.

Proposition 2.7. To give an object, $|H|$, in $\mathcal{D}$ the structure of a $\mathcal{V}$-algebra object is equivalent to giving a lift of the contravariant hom-functor $\mathcal{D}(-,|H|): \mathcal{D} \rightarrow$ Set to a functor $\mathcal{D} \rightarrow \mathcal{V}$. 
To get free $\mathcal{V}$-algebra objects we need to know that we can "impose" identities on an $\Omega$-algebra object.

Theorem 2.8. Let $\mathcal{D}$ be a complete category. Suppose that $\mathcal{D}$ is an $(\mathcal{E}, \mathcal{M})$ category for some classes $\mathcal{E}$ of epimorphisms and $\mathcal{M}$ of monomorphisms, that $\mathcal{E}$ is closed under taking finite products, and that $\mathcal{D}$ is $\mathcal{E}$-co-well-powered. Then the inclusion functor $\mathcal{D} \mathcal{V} \rightarrow \mathcal{D} \Omega$ has a left adjoint, $\mathcal{D} \Omega \rightarrow \mathcal{D} \mathcal{V}$, called imposition of identities.

Corollary 2.9. Under the conditions of Theorem 2.8 and Proposition 2.3, the forgetful functor $\mathcal{D} \mathcal{V} \rightarrow \mathcal{D}$ has a left adjoint, $\mathfrak{F}_{\mathcal{V}}: \mathcal{D} \rightarrow \mathcal{D V}$, which is called the free $\mathcal{V}$-algebra functor.

Dual to $\mathcal{V}$-algebra objects are co- $\mathcal{V}$-algebra objects.

Definition 2.10. Let $\mathcal{V}$ be a variety of algebras, $\mathcal{D}$ a category with finite co-products.

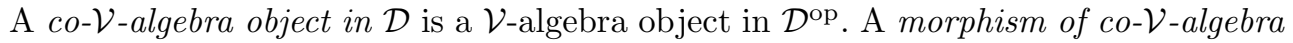
objects in $\mathcal{D}$ is a morphism in $\mathcal{D}$ which intertwines the co- $\mathcal{V}$-algebra structures. We denote the category of co- $\mathcal{V}$-algebra objects in $\mathcal{D}$ by $\mathcal{D} \mathcal{V}^{c}$.

The morphisms are chosen such that there is an isomorphism of categories

$$
\mathcal{D} \mathcal{V}^{c} \cong\left(\mathcal{D}^{\mathrm{op}} \mathcal{V}\right)^{\mathrm{op}}
$$

and there is a natural forgetful functor $\mathcal{D} \mathcal{V}^{c} \rightarrow \mathcal{D}$. The analogue of Proposition 2.2 is the following.

Proposition 2.11. To give an object, $|G|$, in $\mathcal{D}$ the structure of a co-V-algebra object is equivalent to giving a lift of the covariant hom-functor $\mathcal{D}(|G|,-): \mathcal{D} \rightarrow$ Set to a functor $\mathcal{D} \rightarrow \mathcal{V}$.

We shall use similar notation: $\mathcal{D}(G, D)$ will denote the $\mathcal{V}$-algebra with underlying set $\mathcal{D}(|G|, D)$.

The main tool of our analysis is the link between representable functors and functors with adjoints. This result is a standard one from general algebra, although one of its corollaries is perhaps the best known result.

Theorem 2.12. Let $\mathcal{D}$ be a category that has finite products, is co-complete, is cowell-powered, is an $(\mathcal{E}, \mathcal{M})$ category where $\mathcal{E}$ is closed under finite products, and is such that its finite products commute with filtered co-limits. Let $\mathcal{V}$ be a variety of algebras. Let $\mathcal{F}$ be a category with co-equalisers. Let $\mathfrak{G}: \mathcal{F} \rightarrow \mathcal{D V}$ be a covariant functor. Then the following statements are equivalent.

1. $\mathfrak{G}$ has a left adjoint, $\mathfrak{G}_{\text {! }}$.

2. The composition $|\mathfrak{G}|: \mathcal{F} \rightarrow \mathcal{D}$ of $\mathfrak{G}$ with the forgetful functor $\mathcal{D} \mathcal{V} \rightarrow \mathcal{D}$ has a left adjoint, $|\mathfrak{G}|_{!}$.

The relationship between the two adjoints is that there is a co-equaliser sequence in $\mathcal{F}$, natural in $R$,

$$
|\mathfrak{G}|_{!}(|\mathfrak{F} \mathcal{V}(|R|)|) \stackrel{r_{R}}{\underset{s_{R}}{\gtrless}}|\mathfrak{G}|_{!}(|R|) \stackrel{p_{R}}{\longrightarrow} \mathfrak{G}_{!}(R) .
$$

Working in the opposite category we obtain the corresponding result on co-algebra objects. 
Corollary 2.13. Let $\mathcal{D}$ be a category that has finite products, is co-complete, is cowell-powered, is an $(\mathcal{E}, \mathcal{M})$ category where $\mathcal{E}$ is closed under finite products, and its finite products commute with filtered co-limits. Let $\mathcal{V}$ be a variety of algebras. Let $\mathcal{F}$ be a category with equalisers. Let $\mathfrak{G}: \mathcal{F} \rightarrow \mathcal{D V}$ be a contravariant functor. Then the following statements are equivalent.

1. $\mathfrak{G}$ is one of a mutually right adjoint pair.

2. The composition $|\mathfrak{G}|: \mathcal{F} \rightarrow \mathcal{D}$ of $\mathfrak{G}$ with the forgetful functor $\mathcal{D} \mathcal{V} \rightarrow \mathcal{D}$ is one of a mutually right adjoint pair.

Since a functor from a co-complete category into Set is representable if and only if it has a left adjoint, the following standard result of general algebra readily follows from Proposition 2.11.

Corollary 2.14. 1. Let $\mathcal{F}$ be a co-complete category, $\mathcal{V}$ a variety of algebras. For a covariant functor $\mathfrak{G}: \mathcal{F} \rightarrow \mathcal{V}$, the following statements are equivalent.

(a) $\mathfrak{G}$ has a left adjoint.

(b) $\mathfrak{G}$ is representable by a co-V-algebra object in $\mathcal{F}$.

(c) $|\mathfrak{G}|$ is representable by an object in $\mathcal{F}$.

2. Let $\mathcal{F}$ be a complete category, $\mathcal{V}$ a variety of algebras. For a contravariant functor $\mathfrak{G}: \mathcal{F} \rightarrow \mathcal{V}$, the following statements are equivalent.

(a) $\mathfrak{G}$ is one of a mutually right adjoint pair.

(b) $\mathfrak{G}$ is representable by a $\mathcal{V}$-algebra object in $\mathcal{F}$.

(c) $|\mathfrak{G}|$ is representable by an object in $\mathcal{F}$.

We shall need to know various categorical properties of $\mathcal{V}$.

Theorem 2.15. As a category, $\mathcal{V}$ is complete, co-complete, well-powered, extremally co-well-powered, and is an (extremal epi, mono) category.

A morphism is an extremal epimorphism if and only if the underlying morphism of sets is an epimorphism. Moreover, all extremal epimorphisms are regular epimorphisms.

\subsection{Graded Algebras}

We turn now to graded algebras. A graded algebra has components indexed by some (fixed) set and its operations go from components to components rather than being globally defined. The theory of graded algebras is very similar to that of ungraded algebras.

We fix some (non-empty) grading set $Z$. We shall regard this both as a set and as a (small) discrete category. We write $\mathcal{D}^{Z}$ for the category of functors $Z \rightarrow \mathcal{D}$. As $Z$ is a discrete category, there is no distinction between covariant and contravariant functors from $Z$.

An object, $D$, in $\mathcal{D}^{Z}$ represents both a covariant and a contravariant functor $\mathcal{D} \rightarrow$ Set $^{Z}$ via

$$
\begin{aligned}
& D_{*}\left(D^{\prime}\right)=\left(z \mapsto \mathcal{D}\left(D(z), D^{\prime}\right)\right), \\
& D^{*}\left(D^{\prime}\right)=\left(z \mapsto \mathcal{D}\left(D^{\prime}, D(z)\right)\right) .
\end{aligned}
$$

To define a graded algebra, we first need to define the graded version of a type. 
Definition 2.16. A $Z$-graded type, $\Omega^{*}$, is a triple $\left(\left|\Omega^{*}\right|, i, o\right)$ where $\left|\Omega^{*}\right|$ is a set, and

$$
i:\left|\Omega^{*}\right| \rightarrow \coprod_{m \in \mathbb{N}_{0}} Z^{m} \quad \text { and } \quad o:\left|\Omega^{*}\right| \rightarrow Z
$$

are morphisms of sets. For an operation $\omega$, we call $i(\omega)$ the input and $o(\omega)$ the output of $\omega$. We define the arity function, $n:\left|\Omega^{*}\right| \rightarrow \mathbb{N}_{0}$ by the composition

$$
\left|\Omega^{*}\right| \stackrel{i}{\rightarrow} \coprod_{m \in \mathbb{N}_{0}} Z^{m} \rightarrow \coprod_{m \in \mathbb{N}_{0}}\{*\} \cong \mathbb{N}_{0}
$$

We think of $\coprod_{m \in \mathbb{N}_{0}} Z^{m}$ as the set of finite ordered sets of elements of $Z$ and so we interpret the element in $Z^{0}$ as representing the empty subset of $Z$. Under the assumption that $\mathcal{D}$ has finite products, for an element $\left(z_{1}, \ldots, z_{m}\right)$ and an object, $D$, in $\mathcal{D}^{Z}$, we write

$$
D\left(z_{1}, \ldots, z_{m}\right)=\prod_{j=1}^{m} D\left(z_{j}\right)
$$

with $D(\emptyset)=T_{\mathcal{D}}$, the terminal object in $\mathcal{D}$.

Definition 2.17. Let $\mathcal{D}$ be a category with finite products.

1. An $\Omega^{*}$-algebra object, $H$, in $\mathcal{D}$ consists of an object, $|H|$, in $\mathcal{D}^{Z}$ together with, for each $\omega \in\left|\Omega^{*}\right|$, a $\mathcal{D}$-morphism

$$
\omega_{H}:|H|(i(\omega)) \rightarrow|H|(o(\omega)) .
$$

A morphism of $\Omega^{*}$-algebra objects in $\mathcal{D}$ is a morphism of the underlying objects in $\mathcal{D}^{Z}$ which intertwines the operations.

2. An $\Omega^{*}$-algebra is an $\Omega^{*}$-algebra object in Set.

3. We denote the category of $\Omega^{*}$-algebra objects in $\mathcal{D}$ by $\mathcal{D} \Omega^{*}$ and the category of $\Omega^{*}$-algebra objects in Set by $\Omega^{*}$. We refer to the functor $\mathcal{D} \Omega^{*} \rightarrow \mathcal{D}^{Z}$ which assigns to an $\Omega^{*}$-algebra object in $\mathcal{D}$ the underlying object in $\mathcal{D}^{Z}$ as the forgetful functor. We write the underlying object in $\mathcal{D}^{Z}$ of an $\Omega^{*}$-algebra object, $H$, in $\mathcal{D}$ as $|H|$.

All of the results for ungraded algebras have their counterparts in graded algebras.

Proposition 2.18. To give an object, $|H|$, in $\mathcal{D}^{Z}$ the structure of an $\Omega^{*}$-algebra object is equivalent to giving a lift of the contravariant hom-functor $\mathcal{D}(-,|H|): \mathcal{D} \rightarrow$ $\mathrm{Set}^{Z}$ to a functor $\mathcal{D} \rightarrow \Omega^{*}$.

As before, if $H$ is an $\Omega^{*}$-algebra object in $\mathcal{D}$ and $D$ is an object in $\mathcal{D}$ we shall write

$$
\mathcal{D}(D, H)
$$

for the corresponding $\Omega^{*}$-algebra with underlying object in $\operatorname{Set}^{Z}$,

$$
z \mapsto \mathcal{D}(D,|H|(z)) .
$$

Free $\Omega^{*}$-algebras exist under suitable circumstances. 
Proposition 2.19. Let $\mathcal{D}$ be a category with finite products which is closed under filtered co-limits and such that finite products commute with filtered co-limits. Then the forgetful functor $\mathcal{D} \Omega^{*} \rightarrow \mathcal{D}^{Z}$ has a left adjoint, $\mathfrak{F}_{\Omega^{*}}: \mathcal{D}^{Z} \rightarrow \mathcal{D} \Omega^{*}$, which is called the free $\Omega^{*}$-algebra functor.

Identities are defined by modifying the ungraded definition in the obvious way.

Definition 2.20. Let $\Omega^{*}$ be a graded type. An identity for $\Omega^{*}$-algebras is a triple $(X, u, v)$ where $X$ is an object in $\operatorname{Set}^{Z}$ such that $\coprod_{z \in Z} X(z)$ is a finite set and $u, v \in$ $\left|\mathfrak{F}_{\Omega^{*}}(X)\right|(z)$ for some $z \in Z$.

Let $(X, u, v)$ be an identity for $\Omega^{*}$-algebras with $u, v \in\left|\mathfrak{F}_{\Omega^{*}}(X)\right|\left(z_{1}\right)$. Let $H$ be an $\Omega^{*}$-algebra object in $\mathcal{D}$. Consider the object in $\operatorname{Set}^{Z}$

$$
z_{0} \mapsto \mathcal{D}\left(\prod_{z \in Z}|H|(z)^{X(z)},|H|\left(z_{0}\right)\right) .
$$

As $\coprod_{z \in Z} X(z)$ is finite, the product on the left is finite. For each $z_{0} \in Z$, there is an obvious projection morphism in $\mathcal{D}$

$$
\prod_{z \in Z}|H|(z)^{X(z)} \rightarrow|H|\left(z_{0}\right)^{X\left(z_{0}\right)}
$$

and thus for $x \in X\left(z_{0}\right)$ we have a canonical projection morphism in $\mathcal{D}$

$$
\prod_{z \in Z}|H|(z)^{X(z)} \rightarrow|H|\left(z_{0}\right) .
$$

This yields a Set morphism

$$
X\left(z_{0}\right) \rightarrow \mathcal{D}\left(\prod_{z \in Z}|H|(z)^{X(z)},|H|\left(z_{0}\right)\right)
$$

and thus a natural transformation of functors, equivalently a $\operatorname{Set}^{Z}$-morphism,

$$
X \rightarrow \mathcal{D}\left(\prod_{z \in Z}|H|(z)^{X(z)},|H|\right) .
$$

The same adjunction argument as in the ungraded case now produces $\mathcal{D}$-morphisms

$$
u_{H}, v_{H}: \prod_{z \in Z}|H|(z)^{X(z)} \rightarrow|H|\left(z_{1}\right) .
$$

Definition 2.21. An $\Omega^{*}$-algebra object, $H$, in $\mathcal{D}$ is said to satisfy the identity $(X, u, v)$ if the two induced morphisms $u_{H}, v_{H}$ are the same.

Definition 2.22. A variety of graded algebras, $\mathcal{V}^{*}$, is specified by a graded type, $\Omega^{*}$, and a set of identities for $\Omega^{*}$-algebras, $J$. It is the full subcategory of $\Omega^{*}$ consisting of all $\Omega^{*}$-algebras which satisfy all of the identities in $J$.

The pair $\left(\Omega^{*}, J\right)$ is a presentation of $\mathcal{V}^{*}$.

Let $\mathcal{D}$ be a category with finite products, $\mathcal{V}^{*}$ a variety of graded algebras with presentation $\left(\Omega^{*}, J\right)$. The category of $\mathcal{V}^{*}$-algebra objects in $\mathcal{D}, \mathcal{D} \mathcal{V}^{*}$, is the full subcategory of $\mathcal{D} \Omega^{*}$ consisting of all $\Omega^{*}$-algebra objects in $\mathcal{D}$ which satisfy all of the identities in $J$. 
Proposition 2.18 holds in the presence of identities.

Proposition 2.23. To give an object, $|H|$, in $\mathcal{D}^{Z}$ the structure of a $\mathcal{V}^{*}$-algebra object is equivalent to giving a lift of the contravariant hom-functor $\mathcal{D}(-,|H|): \mathcal{D} \rightarrow \operatorname{Set}^{Z}$ to a functor $\mathcal{D} \rightarrow \mathcal{V}^{*}$.

The same conditions as in the ungraded case allow us to impose identities and so get free $\mathcal{V}^{*}$-algebra objects.

Theorem 2.24. Let $\mathcal{D}$ be a complete category. Suppose that $\mathcal{D}$ is an $(\mathcal{E}, \mathcal{M})$ category for some classes $\mathcal{E}$ of epimorphisms and $\mathcal{M}$ of monomorphisms, that $\mathcal{E}$ is closed under taking finite products, and that $\mathcal{D}$ is $\mathcal{E}$-co-well-powered. Then the inclusion functor $\mathcal{D} \mathcal{V}^{*} \rightarrow \mathcal{D} \Omega^{*}$ has a left adjoint, $\mathcal{D} \Omega^{*} \rightarrow \mathcal{D} \mathcal{V}^{*}$, called imposition of identities.

Corollary 2.25. Under the conditions of Theorem 2.24 and Proposition 2.19, the forgetful functor $\mathcal{D V}^{*} \rightarrow \mathcal{D}^{Z}$ has a left adjoint, $\mathfrak{F}_{\mathcal{V}^{*}}: \mathcal{D}^{Z} \rightarrow \mathcal{D} \mathcal{V}^{*}$, which is called the free $\mathcal{V}^{*}$-algebra functor.

Dual to $\mathcal{V}^{*}$-algebra objects are co- $\mathcal{V}^{*}$-algebra objects.

Definition 2.26. Let $\mathcal{V}^{*}$ be a variety of graded algebras, $\mathcal{D}$ a category with finite coproducts. A co- $\mathcal{V}^{*}$-algebra object in $\mathcal{D}$ is a $\mathcal{V}^{*}$-algebra object in $\mathcal{D}^{\mathrm{op}}$. A morphism of co- $\mathcal{V}^{*}$-algebra objects in $\mathcal{D}$ is a morphism in $\mathcal{D}^{Z}$ which intertwines the co- $\mathcal{V}^{*}$-algebra object in $\mathcal{D}$ structures. We denote the category of co- $\mathcal{V}^{*}$-algebra objects in $\mathcal{D}$ by $\mathcal{D V} \mathcal{V}^{* c}$.

The analogue of Proposition 2.18 is the following.

Proposition 2.27. To give an object, $|G|$, in $\mathcal{D}^{Z}$ the structure of a co- $\mathcal{V}^{*}$-algebra object is equivalent to giving a lift of the covariant hom-functor $\mathcal{D}(|G|,-): \mathcal{D} \rightarrow \operatorname{Set}^{Z}$ to a functor $\mathcal{D} \rightarrow \mathcal{V}^{*}$.

We shall use similar notation: $\mathcal{D}(G, D)$ will denote the $\mathcal{V}^{*}$-algebra with underlying object $\mathcal{D}(|G|, D)$ in $\operatorname{Set}^{Z}$.

Theorem 2.12 easily generalises to the graded case.

Theorem 2.28. Let $\mathcal{D}$ be a category that has finite products, is co-complete, is cowell-powered, is an $(\mathcal{E}, \mathcal{M})$ category where $\mathcal{E}$ is closed under finite products, and its finite products commute with filtered co-limits. Let $\mathcal{V}^{*}$ be a variety of graded algebras. Let $\mathcal{F}$ be a category with co-equalisers. Let $\mathfrak{G}: \mathcal{F} \rightarrow \mathcal{D V}^{*}$ be a covariant functor. Then the following statements are equivalent.

1. $\mathfrak{G}$ has a left adjoint, $\mathfrak{G}_{!}$.

2. The composition $|\mathfrak{G}|: \mathcal{F} \rightarrow \mathcal{D}^{Z}$ of $\mathfrak{G}$ with the forgetful functor $\mathcal{D V}^{*} \rightarrow \mathcal{D}^{Z}$ has a left adjoint, $|\mathfrak{G}|_{!}$.

We have the same relationship between the two adjoints as in the ungraded case: there is a co-equaliser sequence in $\mathcal{F}$, natural in $H$,

$$
|\mathfrak{G}|_{!}\left(\left|\mathfrak{F} \mathcal{V}^{*}(|H|)\right|\right) \underset{s_{H}}{\stackrel{r_{H}}{\longrightarrow}}|\mathfrak{G}|_{!}(|H|) \stackrel{p_{H}}{\longrightarrow} \mathfrak{G}_{!}(H) .
$$

The graded version of Corollary 2.13 follows immediately. To get the graded version of Corollary 2.14 we need to understand the relationship between adjunctions and representability in the graded case. 
Lemma 2.29. Let $\mathcal{D}$ be a co-complete category. A covariant functor $\mathfrak{G}: \mathcal{D} \rightarrow \operatorname{Set}^{Z}$ has a left adjoint if and only if it is representable by an object in $\mathcal{D}^{Z}$.

Proof. Suppose that $\mathfrak{G}$ has a left adjoint, say $\mathfrak{H}: \operatorname{Set}^{Z} \rightarrow \mathcal{D}$. We extend this to a functor $\mathfrak{H}^{Z}:\left(\operatorname{Set}^{Z}\right)^{Z} \rightarrow \mathcal{D}^{Z}$ in the obvious way. Let $I$ be the object in $\left(\operatorname{Set}^{Z}\right)^{Z}$ defined by

$$
z \mapsto\left(z^{\prime} \mapsto\left\{\begin{array}{ll}
\{*\} & \text { if } z=z^{\prime} \\
\emptyset & \text { otherwise }
\end{array}\right) .\right.
$$

Then for an object, $X$, in $\operatorname{Set}^{Z}$ we have a natural isomorphism $\operatorname{Set}^{Z}(I, X) \cong X$ of objects in $\operatorname{Set}^{Z}$. Hence for $D$ an object in $\mathcal{D}$ there are natural isomorphisms

$$
\mathfrak{G}(D) \cong \operatorname{Set}^{Z}(I, \mathfrak{G}(D)) \cong \mathcal{D}\left(\mathfrak{H}^{Z}(I), D\right)
$$

and so $\mathfrak{G}$ is represented by the object, $\mathfrak{H}^{Z}(I)$, in $\mathcal{D}^{Z}$.

Conversely, suppose that $\mathfrak{G}$ is represented by the object, $G$, in $\mathcal{D}^{Z}$. We define the functor $\mathfrak{H}: \operatorname{Set}^{Z} \rightarrow \mathcal{D}$ on objects by

$$
\mathfrak{H}(X)=\coprod_{z \in Z} \coprod_{X(z)} G(z)
$$

and in the obvious way on morphisms. It is straightforward to show that this is the required left adjoint.

As a corollary we deduce the graded version of Corollary 2.14.

\section{Corollary 2.30.}

1. Let $\mathcal{D}$ be a co-complete category, $\mathcal{V}^{*}$ a variety of graded algebras. For a covariant functor $\mathfrak{G}: \mathcal{D} \rightarrow \mathcal{V}^{*}$, the following statements are equivalent.

(a) $\mathfrak{G}$ has a left adjoint.

(b) $\mathfrak{G}$ is representable by a co- $\mathcal{V}^{*}$-algebra object in $\mathcal{D}$.

(c) $|\mathfrak{G}|$ is representable by an object in $\mathcal{D}^{Z}$.

2. Let $\mathcal{D}$ be a complete category, $\mathcal{V}^{*}$ a variety of graded algebras. For a contravariant functor $\mathfrak{G}: \mathcal{D} \rightarrow \mathcal{V}^{*}$, the following statements are equivalent.

(a) $\mathfrak{G}$ is part of a mutually right adjoint pair.

(b) $\mathfrak{G}$ is representable by an $\mathcal{V}^{*}$-algebra object in $\mathcal{D}$.

(c) $|\mathfrak{G}|$ is representable by an object in $\mathcal{D}^{Z}$.

The categorical properties of $\mathcal{V}^{*}$ are the same as those of $\mathcal{V}$.

Theorem 2.31. As a category, $\mathcal{V}^{*}$ is complete, co-complete, well-powered, extremally co-well-powered, and is an (extremal epi, mono) category.

A morphism is an extremal epimorphism if and only if the underlying morphism of objects in $\mathrm{Set}^{Z}$ is an epimorphism. Moreover, all extremal epimorphisms are regular epimorphisms.

The work of the following sections can be viewed simply as applications of Corollaries 2.14 and 2.30 . 


\subsection{The Tall-Wraith Monoidal Structure}

The categories $\mathcal{V} \mathcal{V}^{c}$ and $\mathcal{V}^{*} \mathcal{V}^{* c}$ have a monoidal structure corresponding to composition of (representable) functors. The first trace of this that we have discovered in the literature is [Fre66] where it is referred to as the tensor product of algebras. The first study of the corresponding monoids that we have found is [TW70] which deals with the category of commutative, unital rings. As we are similarly interested in the monoids, we have elected to call them Tall-Wraith monoids. For consistency, and because the terminology of tensor products is already somewhat overused, we name the monoidal structure on $\mathcal{V} \mathcal{V}^{c}$ and $\mathcal{V}^{*} \mathcal{V}^{* c}$ the Tall-Wraith monoidal structure.

Although, as we have just said, the ideas in this section go back at least to [Fre66], we have not been able to find a reference which covers all that we wish to do; in particular, Theorem 2.34 and the extensions to graded algebras. On the other hand, these results are not central to this paper but rather are a guide to what to expect in the filtered context and so we have not included their proofs here. The missing proofs can be found in [SW09].

In addition to [Fre66] and [TW70] mentioned above, similar ideas occur in [BW05] and [BH96].

Theorem 2.32. Let $\mathcal{V}$ be a variety of algebras. The category $\mathcal{V V}^{c}$ has a monoidal structure which we write as $\left(\mathcal{V} \mathcal{V}^{c}, \odot, I\right)$. The functor $\mathcal{V} \mathcal{V}^{c} \rightarrow \operatorname{CovFun}(\mathcal{V}, \mathcal{V})$ given by sending a co-V-algebra object in $\mathcal{V}$ to the covariant functor that it represents, is strong monoidal. The underlying object in $\mathcal{V}$ of the unit, $I$, is isomorphic to $\mathfrak{F}_{\mathcal{V}}(\{*\})$.

We shall not give a full proof of this result here; the idea can be found in [Fre66] and a full proof is in [SW09]. We shall give a description of the product pairing as this will be important later.

For a co- $\mathcal{V}$-algebra object, $B$, in $\mathcal{V}$ let us write $B_{*}: \mathcal{V} \rightarrow \mathcal{V}$ for the covariant functor that it represents. By Corollary 2.14, this functor has a left adjoint which we denote by $B_{!}$. Now co- $\mathcal{V}$-algebra objects in $\mathcal{V}$ are objects in $\mathcal{V}$ with extra structure; this extra structure involves morphisms from the underlying object in $\mathcal{V}$ to iterated co-products of it. As $B_{\text {! }}$ is a left adjoint, it preserves co-products and thus lifts to a functor $B_{!}^{c}: \mathcal{V} \mathcal{V}^{c} \rightarrow \mathcal{V} \mathcal{V}^{c}$. The assignment $B \mapsto B_{!}^{c}$ is functorial in $B$. The pairing on $\mathcal{V} \mathcal{V}^{c}$ is, up to natural isomorphism, given on objects by

$$
\left(B_{1}, B_{2}\right) \mapsto B_{2 !}^{c}\left(B_{1}\right) .
$$

It has the property that we have natural isomorphisms

$$
\mathcal{V}\left(B_{1}, \mathcal{V}\left(B_{2}, B_{3}\right)\right) \cong \mathcal{V}\left(B_{1} \odot B_{2}, B_{3}\right) .
$$

Theorem 2.32 readily adapts to the following situations.

Proposition 2.33. Let $\mathcal{D}$ be a co-complete category, $\mathcal{V}$ and $\mathcal{W}$ varieties of algebras. There are products

$$
\begin{aligned}
\mathcal{V} \mathcal{V}^{c} \times \mathcal{D} \mathcal{V}^{c} & \rightarrow \mathcal{D} \mathcal{V}^{c}, \\
\left(\mathcal{V} \mathcal{V}^{c}\right)^{\mathrm{op}} \times \mathcal{D} \mathcal{V} & \rightarrow \mathcal{D} \mathcal{V}, \\
\mathcal{V} \mathcal{W}^{c} \times \mathcal{V} \mathcal{V}^{c} & \rightarrow \mathcal{V} \mathcal{W}^{c},
\end{aligned}
$$

all compatible with the monoidal structure of $\mathcal{V V}^{c}$ and with composition of representable functors. 
We write all of the pairings using the notation $-\odot-$.

There are two things to note from this generalisation. Firstly that there are two pairings which involve $\mathcal{V} \mathcal{V}^{c}$ and $\mathcal{V}$. The first views $\mathcal{V}$ as Set $\mathcal{V}$ and so comes from the middle pairing above with $\mathcal{D}=$ Set; in terms of functors we have

$$
(B \odot V)^{*}(X)=\operatorname{Set}(X, B \odot V) \cong \mathcal{V}(B, \operatorname{Set}(X, V)) .
$$

The second views $\mathcal{V}$ as $\mathcal{V}$ Set $^{c}$ and so comes from the third pairing with $\mathcal{W}=$ Set; in terms of functors we have

$$
(V \odot B)_{*}\left(V^{\prime}\right)=\mathcal{V}\left(V \odot B, V^{\prime}\right) \cong \mathcal{V}\left(V, \mathcal{V}\left(B, V^{\prime}\right)\right) .
$$

This latter pairing was the one considered in $[\mathbf{T W 7 0}]$ with $\mathcal{V}$ the category of commutative, unital rings.

The second thing to note from this generalisation is the annoyance of having a partially contravariant pairing. Providing $\mathcal{D}$ is sufficiently structured this can be countered; again, the details can be found in [SW09].

Theorem 2.34. Let $\mathcal{D}$ be a category satisfying the conditions of Theorem 2.12. Then there is a pairing

$$
\mathcal{V} \mathcal{V}^{c} \times \mathcal{D} \mathcal{V} \rightarrow \mathcal{D} \mathcal{V}, \quad(B, R) \mapsto B \circledast R,
$$

which is covariant in both arguments and satisfies

$$
\mathcal{D} \mathcal{V}\left(B \circledast R, R^{\prime}\right) \cong \mathcal{D} \mathcal{V}\left(R, B \odot R^{\prime}\right)
$$

naturally in all arguments.

In a monoidal category it is natural to consider monoids.

Definition 2.35. Let $\mathcal{V}$ be a variety of algebras. A Tall-Wraith $\mathcal{V}$-monoid is a monoid in $\mathcal{V} \mathcal{V}^{c}$. We write the category of such monoids as $\mathcal{V} \mathcal{V}^{c} \mathcal{T}{ }^{\odot}$.

These were discussed briefly in [BH96, Ch. 63, 64], though without explicit reference to the underlying monoidal structure on $\mathcal{V} \mathcal{V}^{c}$.

With a monoid one can consider modules for that monoid. Since the monoidal category $\mathcal{V} \mathcal{V}^{c}$ acts on other categories we can consider modules that are not co- $\mathcal{V}$ algebra objects in $\mathcal{V}$. That is, if $P$ is a Tall-Wraith $\mathcal{V}$-monoid and $\mathcal{D}$ is a co-complete category then we can consider co- $\mathcal{V}$-algebra objects, $G$, in $\mathcal{D}$ for which there is a $\mathcal{D} \mathcal{V}^{c}$-morphism

$$
P \odot G \rightarrow G
$$

satisfying the obvious coherences.

In [BH96] the authors show that the category of objects in $\mathcal{V}$ with an action of a Tall-Wraith $\mathcal{V}$-monoid is again a variety of algebras. Extending this, we easily see that a $\mathcal{V}$-algebra object in $\mathcal{D}$ or co- $\mathcal{V}$-algebra object in $\mathcal{D}$ is a module for a Tall-Wraith $\mathcal{V}$ monoid if and only if the corresponding functor $\mathcal{D} \rightarrow \mathcal{V}$ factors through the category of objects in $\mathcal{V}$ with an action of the Tall-Wraith $\mathcal{V}$-monoid.

Two remarks are worth making at this juncture. Firstly, if $\mathcal{W}$ is another variety of algebras then the structure of a $P$-module on a co- $\mathcal{W}$-algebra object in $\mathcal{V}$ does not have such an interpretation since a co- $\mathcal{W}$-algebra object in $\mathcal{V}$ represents a functor out 
of $\mathcal{V}$. Secondly, due to the variance shift, a $P$-module in $\mathcal{D V}$ is better thought of as a $P$-co-module as the required morphism is

$$
R \rightarrow P \odot R .
$$

We can surmount this using the product $\circledast$ since the adjunction turns a co-action as above into a more normal-looking action. That is to say, if a $\mathcal{V}$-algebra object, $R$, in $\mathcal{D}$ is a $P$-co-module for $\odot$ with co-action morphism

$$
R \rightarrow P \odot R
$$

then it is a $P$-module for $\circledast$ with action morphism

$$
P \circledast R \rightarrow R
$$

where the action and co-action morphisms correspond under the isomorphism

$$
\mathcal{D} \mathcal{V}(P \circledast R, R) \cong \mathcal{D} \mathcal{V}(R, P \odot R) .
$$

The adaptation of all this to the graded situation is straightforward.

Theorem 2.36. Let $\mathcal{V}^{*}$ be a variety of graded algebras. The category $\mathcal{V}^{*} \mathcal{V}^{* c}$ has a monoidal structure which we write as $\left(\mathcal{V}^{*} \mathcal{V}^{* c}, \odot, I\right)$. Moreover, the functor $\mathcal{V}^{*} \mathcal{V}^{* c} \rightarrow \operatorname{CovFun}\left(\mathcal{V}^{*}, \mathcal{V}^{*}\right)$, given by sending a co- $\mathcal{V}^{*}$-algebra object in $\mathcal{V}^{*}$ to the covariant functor that it represents, is strong monoidal.

As before we shall give a description of the product. A co- $\mathcal{V}^{*}$-algebra object, $B$, in $\mathcal{V}^{*}$ represents a functor $B_{*}: \mathcal{V}^{*} \rightarrow \mathcal{V}^{*}$. By Corollary 2.30 this functor has a left adjoint $B_{!}: \mathcal{V}^{*} \rightarrow \mathcal{V}^{*}$. We extend this to a functor $B_{!}^{Z}: \mathcal{V}^{* Z} \rightarrow \mathcal{V}^{* Z}$ in the obvious way. Co-products in a graded category are formed by taking component-by-component coproducts, whence $B_{!}^{Z}$ preserves co-products because $B_{!}$does. Hence it lifts to a functor $B_{!}^{c}: \mathcal{V}^{*} \mathcal{V}^{* c} \rightarrow \mathcal{V}^{*} \mathcal{V}^{* c}$. This has the property that the adjunction isomorphism lifts to an isomorphism of $\mathcal{V}^{*}$-algebras

$$
\mathcal{V}^{*}\left(B_{1}, \mathcal{V}^{*}\left(B_{2}, V\right)\right) \cong \mathcal{V}^{*}\left(B_{2 !}^{c}\left(B_{1}\right), V\right) .
$$

Thus there is a natural isomorphism of co- $\mathcal{V}^{*}$-algebra objects in $\mathcal{V}^{*}$

$$
B_{1} \odot B_{2} \cong B_{2 !}^{c}\left(B_{1}\right) \text {. }
$$

The other part of the structure that needs a word of explanation is the representing object for the unit of the monoidal structure. We saw in the proof of Lemma 2.29 that the identity functor $\operatorname{Set}^{Z} \rightarrow \operatorname{Set}^{Z}$ is representable by an object in $\left(\operatorname{Set}^{Z}\right)^{Z}$, labelled $I$ in that proof. The free $\mathcal{V}^{*}$-algebra on the components of this object in $\left(\operatorname{Set}^{Z}\right)^{Z}$ represents the identity on $\mathcal{V}^{*}$.

Proposition 2.37. Let $\mathcal{D}$ be a co-complete category, $\mathcal{V}^{*}$ and $\mathcal{W}^{*}$ varieties of graded algebras. There are products

$$
\begin{aligned}
\mathcal{V}^{*} \mathcal{V}^{* c} \times \mathcal{D} \mathcal{V}^{* c} & \rightarrow \mathcal{D} \mathcal{V}^{* c}, \\
\left(\mathcal{V}^{*} \mathcal{V}^{* c}\right)^{\text {op }} \times \mathcal{D} \mathcal{V}^{*} & \rightarrow \mathcal{D} \mathcal{V}^{*}, \\
\mathcal{V}^{*} \mathcal{W}^{* c} \times \mathcal{V}^{*} \mathcal{V}^{* c} & \rightarrow \mathcal{V}^{*} \mathcal{W}^{* c},
\end{aligned}
$$

all compatible with the monoidal structure of $\mathcal{V}^{*} \mathcal{V}^{* c}$ and with composition of representable functors. 
We write all of the pairings using the notation $-\odot-$.

We remark that the varieties of graded algebras, $\mathcal{V}^{*}$ and $\mathcal{W}^{*}$, could be graded by different indexing sets. This allows us to take, for example, $\mathcal{W}^{*}=$ Set and so get the obvious pairing

$$
\mathcal{V}^{*} \times \mathcal{V}^{*} \mathcal{V}^{* c} \rightarrow \mathcal{V}^{*}
$$

We can remove the variance switch in the middle product by means of the graded analogue of Theorem 2.34.

Theorem 2.38. Let $\mathcal{D}$ be a category satisfying the conditions of Theorem 2.12. Then there is a pairing

$$
\mathcal{V}^{*} \mathcal{V}^{* c} \times \mathcal{D} \mathcal{V}^{*} \rightarrow \mathcal{D} \mathcal{V}^{*}
$$

which is covariant in both arguments and satisfies

$$
\mathcal{D V}^{*}\left(B \circledast H, H^{\prime}\right) \cong \mathcal{D V}^{*}\left(H, B \odot H^{\prime}\right)
$$

naturally in all arguments.

Definition 2.39. Let $\mathcal{V}^{*}$ be a variety of graded algebras. A Tall-Wraith $\mathcal{V}^{*}$-monoid is a monoid in $\mathcal{V}^{*} \mathcal{V}^{* c}$. We write the category of such monoids as $\mathcal{V}^{*} \mathcal{V}^{* c} \mathcal{T}^{\odot}$.

The remarks regarding modules (and co-modules) for a Tall-Wraith $\mathcal{V}$-monoid carry over to the graded case.

\section{Filtered Categories}

The purpose of this section is to introduce a categorical version of a very specific type of topology. What we wish to generalise is the following situation: we have a topological space whose topology is the projective topology for a family of maps into discrete spaces. This particular case is easy to put into a general categorical situation and we do not need any of the usual machinery used to meld topology and category theory.

In the first part we introduce the basic idea: filtered objects. To give a set, $X$, a topology in this fashion, it is sufficient to give a family of maps with source $X$. Putting this into a categorical context leads to projectively filtered objects in an arbitrary category. We shall also define inductively filtered objects since we shall need to consider how contravarient functors transform filtered objects in one category into filtered objects in another category.

In the example of topological spaces, we only need to consider surjective morphisms and we can reduce an arbitrary filtration to one in which all the morphisms are surjective. We cannot mirror this reduction in all categories and, moreover, the condition that a functor preserve epimorphisms is more restrictive than we wish to impose. However, in certain categories there is a reduction functor and we shall examine the extra features of the theory that this introduces.

When we can consider these reduced filtrations it makes sense to consider variations on the themes of being complete and being Hausdorff. Completion is not a purely topological concept, rather it is a notion from the theory of uniform spaces. The correct generalisation of these two notions to reduced filtered objects involves 
examining the projective limit of the filtration. There is a morphism from the underlying object to this limit and we can ask whether this morphism is a monomorphism, epimorphism, or isomorphism. Being a monomorphism corresponds to the topology being Hausdorff whilst being an epimorphism generalises the notion of completeness.

We start by introducing the most general form of filtrations before moving on to the reduced version. Once we have that then we can consider the projective limit.

\subsection{Projective Filtrations}

We start with the general case of a filtration on an object in a category.

Definition 3.1. Let $\mathcal{D}$ be a category, $D$ an object in $\mathcal{D}$. We define $D \downarrow$ to be the quasiordered class whose elements are $\mathcal{D}$-morphisms with source $D$ and whose ordering is given by $d_{1} \geqslant d_{2}$ if there is a $\mathcal{D}$-morphism $h$ such that $h d_{1}=d_{2}$.

A projective filtration, $Q$, on $D$ is a non-empty, saturated, directed subclass of $D \downarrow$.

We say that $Q_{1}$ is stronger than $Q_{2}$ if $Q_{2} \subseteq Q_{1}$.

If we are given a projective filtration $Q$ on an object in $\mathcal{D}$ without having specified the underlying object in $\mathcal{D}$ we shall write it as $|Q|$. An element of $Q$ is a $\mathcal{D}$-morphism which we shall write as $q:|Q| \rightarrow Q_{q}$.

In this context, saturated means that if $d_{1} \geqslant d_{2}$ and $d_{1} \in Q$ then $d_{2} \in Q$.

Let $Q$ be a projective filtration on $D$. Let $f: D^{\prime} \rightarrow D$ be a $\mathcal{D}$-morphism. The family of all elements of $D^{\prime} \downarrow$ of the form $d f$ for $d$ an element of $Q$ is a non-empty, saturated, directed subclass of $D^{\prime} \downarrow$ and hence a projective filtration on $D^{\prime}$.

Definition 3.2. We refer to this filtration as the pull back of $Q$ by $f$ and write it as $f^{*}(Q)$.

This construction is strictly associative.

Lemma 3.3. Given $\mathcal{D}$-morphisms $D_{1} \stackrel{f}{\rightarrow} D_{2} \stackrel{g}{\rightarrow} D_{3}$ and a projective filtration $Q$ on $D_{3}$, the projective filtrations $f^{*}\left(g^{*}(Q)\right)$ and $(g f)^{*}(Q)$ on $D_{1}$ are the same.

With these notions we can define a category of projective filtrations on objects in $\mathcal{D}$.

Definition 3.4. We define the category of projectively filtered objects of $\mathcal{D}, \dot{\mathcal{D}}$. Its objects are projective filtrations on objects in $\mathcal{D}$. A $\overrightarrow{\mathcal{D}}$-morphism $f: Q_{1} \rightarrow Q_{2}$ is a $\mathcal{D}$-morphism $|f|:\left|Q_{1}\right| \rightarrow\left|Q_{2}\right|$ with the property that $Q_{1}$ is stronger than $f^{*}\left(Q_{2}\right)$.

By construction, the obvious functor $\dot{\mathcal{D}} \rightarrow \mathcal{D}$ is faithful.

Any projective filtration is completely determined by an initial subclass, which perforce is directed, and any non-empty directed subclass of $D \downarrow$ determines a projective filtration by saturation; that is, if $d_{1}, d_{2}$ are in $D \downarrow$ with $d_{1} \geqslant d_{2}$ and $d_{1}$ is in the specified class then we include $d_{2}$. It is clear that the original directed class is initial for the resulting projective filtration. If $\mathcal{D}$ has finite products then any subclass of $D \downarrow$, directed or not, determines a projective filtration: first we include all finite products and then we saturate it. Therefore we could choose to work with directed subclasses of $D \downarrow$, or even arbitrary subclasses, but the above formulation of saturated subclasses is most directly analogous to a topology on a set. The correspondences are: projective 
filtration and topology, directed subclass and a basis of the topology, subclass and a subbasis of the topology.

We shall find it useful to have a characterisation of when a $\mathcal{D}$-morphism lifts to a $\overrightarrow{\mathcal{D}}$-morphism in terms of choices of initial subclasses of the projective filtrations involved. Let $Q_{1}$ and $Q_{2}$ be projective filtrations in $\mathcal{D}$ and let $f:\left|Q_{1}\right| \rightarrow\left|Q_{2}\right|$ be a $\mathcal{D}$-morphism on the underlying objects in $\mathcal{D}$. Suppose that we have initial subclasses of $Q_{1}$ and $Q_{2}$ indexed by $\Lambda_{1}$ and $\Lambda_{2}$ respectively. Then $f$ lifts to a $\overrightarrow{\mathcal{D}}$-morphism if and only if for each $\lambda_{2}$ in $\Lambda_{2}$ there is a $\lambda_{1}$ in $\Lambda_{1}$ and a $\mathcal{D}$-morphism $f_{\lambda_{1}, \lambda_{2}}: Q_{1, \lambda_{1}} \rightarrow Q_{2, \lambda_{2}}$ such that the following diagram of $\mathcal{D}$-morphisms commutes

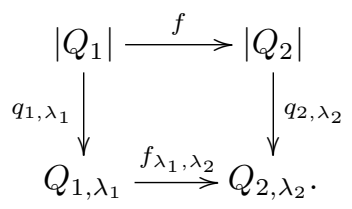

Lemma 3.5. The assignment $\mathcal{D} \rightarrow \overrightarrow{\mathcal{D}}$ underlies a 2-functor of 2-categories Cat $\rightarrow$ Cat.

Proof. For a covariant functor $\mathfrak{G}: \mathcal{D} \rightarrow \mathcal{E}$ we define a covariant functor $\overrightarrow{\mathfrak{G}}: \overrightarrow{\mathcal{D}} \rightarrow \overrightarrow{\mathcal{E}}$ in the obvious way: for $Q$ an object in $\overrightarrow{\mathcal{D}}, \overrightarrow{\mathfrak{G}}(Q)$ is the saturation of the non-empty, directed subclass of $\mathfrak{G}(|Q|) \downarrow$ consisting of $\mathfrak{G}(q)$ for $q \in Q$. For a $\overrightarrow{\mathcal{D}}$-morphism $f: Q_{1} \rightarrow$ $Q_{2}, \overrightarrow{\mathfrak{G}}(f)$ has underlying $\mathcal{E}$-morphism $\mathfrak{G}(|f|)$. That this is an $\overrightarrow{\mathcal{E}}$-morphism is obvious. This construction is compatible with composition in that $\ddot{\mathfrak{G}} \overrightarrow{\mathfrak{H}}=\overrightarrow{\mathfrak{G}} \overrightarrow{\mathfrak{H}}$. It is obvious that identity functors map to identity functors.

Similarly, if $\nu: \mathfrak{G} \rightarrow \mathfrak{H}$ is a natural transformation of covariant functors $\mathcal{D} \rightarrow \mathcal{E}$, we define a natural transformation $\vec{\nu}: \overrightarrow{\mathfrak{G}} \rightarrow \overrightarrow{\mathfrak{H}}$. For an object, $Q$, in $\overrightarrow{\mathcal{D}}$, the $\overrightarrow{\mathcal{E}}-$ morphism $\vec{\nu}_{Q}$ has underlying $\mathcal{E}$-morphism $\nu_{|Q|}$. Again, this construction is obviously compatible with composition and identity natural transformations.

An important consequence of this is the following result.

Proposition 3.6. Let $\mathfrak{G}: \mathcal{D} \rightarrow \mathcal{E}$ be a covariant functor and suppose that it has a left adjoint, say $\mathfrak{H}: \mathcal{E} \rightarrow \mathcal{D}$, then $\overrightarrow{\mathfrak{H}}$ is left adjoint to $\overrightarrow{\mathfrak{G}}$.

If $\mathcal{D}$ has a terminal object then the class of all projective filtrations of a fixed object in $\mathcal{D}$ is a (large!) complete lattice. Its top and bottom elements provide adjoints to the forgetful functor $\overrightarrow{\mathcal{D}} \rightarrow \mathcal{D}$.

Proposition 3.7. The forgetful functor $\overrightarrow{\mathcal{D}} \rightarrow \mathcal{D}$ has a left adjoint $\mathfrak{D}: \mathcal{D} \rightarrow \dot{\mathcal{D}}$. If $\mathcal{D}$ has a terminal object then the forgetful functor $\overrightarrow{\mathcal{D}} \rightarrow \mathcal{D}$ has a right adjoint $\mathfrak{I}: \mathcal{D} \rightarrow \overrightarrow{\mathcal{D}}$.

For an object, $D$, in $\mathcal{D}, \mathfrak{D}(D)$ is $D \downarrow$ whilst $\Im(D)$ is the subclass of $D \downarrow$ consisting of all $\mathcal{D}$-morphisms from $D$ to terminal objects in $\mathcal{D}$.

Proof. Clearly the descriptions given of $\mathfrak{D}(D)$ and $\mathfrak{I}(D)$ do produce projective filtrations on $D$ and if $Q$ is another projective filtration on $D$ then we have $\mathfrak{I}(D) \subseteq Q \subseteq$ $\mathfrak{D}(D)$; the second inclusion by definition and the first as $Q$ is non-empty. 
From this, it is clear that if $f: D_{1} \rightarrow D_{2}$ is a $\mathcal{D}$-morphism then it underlies $\overrightarrow{\mathcal{D}}$ morphisms $\mathfrak{D}\left(D_{1}\right) \rightarrow \mathfrak{D}\left(D_{2}\right)$ and $\mathfrak{I}\left(D_{1}\right) \rightarrow \mathfrak{I}\left(D_{2}\right)$. As the forgetful functor $\overrightarrow{\mathcal{D}} \rightarrow \mathcal{D}$ is faithful, this is sufficient to define $\mathfrak{D}$ and $\mathfrak{I}$ on morphisms.

Clearly, if we apply either $\mathfrak{D}: \mathcal{D} \rightarrow \overrightarrow{\mathcal{D}}$ or $\mathfrak{I}: \mathcal{D} \rightarrow \overrightarrow{\mathcal{D}}$ and the forgetful functor $\overrightarrow{\mathcal{D}} \rightarrow \mathcal{D}$ then the resulting composition is the identity functor on $\mathcal{D}$.

Finally, from above we see that the identity on $D$ lifts to morphisms $\mathfrak{D}(D) \rightarrow Q \rightarrow$ $\mathfrak{I}(D)$. These provide the required natural transformations for the adjunctions.

Definition 3.8. For an object, $D$, in $\mathcal{D}$ we refer to $\mathfrak{D}(D)$ as the discrete (projective) filtration on $D$ and $\mathfrak{I}(D)$ as the indiscrete (projective) filtration on $D$ (assuming that $\mathcal{D}$ has a terminal object).

These two functors are very simple examples of a more general type of functor.

Definition 3.9. To filter a category is to give a functor $\mathcal{D} \rightarrow \overrightarrow{\mathcal{D}}$ which is right inverse to the forgetful functor. We call such a functor a projective filtration functor.

Examples 3.10.

1. The first example is of a pro-finite filtration. Let $\mathcal{F}$ be a non-empty full subcategory of $\mathcal{D}$ which is closed under finite products. We refer to objects in $\mathcal{F}$ as finite objects.

Let $D$ be an object in $\mathcal{D}$. We define a projective filtration on $D$ as follows. We start with the subclass of $D \downarrow$ consisting of all $\mathcal{D}$-morphisms with target a finite object. Our assumption on $\mathcal{F}$ ensures that this is directed. We saturate it to produce a projective filtration.

It is straightforward to show that the assignment to an object in $\mathcal{D}$ of its profinite filtration is functorial. We therefore have the pro-finite filtration functor on $\mathcal{D}$.

2. The second example of a category that can be filtered is that of a filtered category. We shall define a filtration functor

$$
\overrightarrow{\mathcal{D}} \rightarrow \stackrel{: \overrightarrow{\mathcal{D}}}{.}
$$

Let $Q$ be an object in $\overrightarrow{\mathcal{D}}$. We start by observing that for $q \in Q$, the $\mathcal{D}$-morphism $q:|Q| \rightarrow Q_{q}$ is the underlying $\mathcal{D}$-morphism of a $\overrightarrow{\mathcal{D}}$-morphism $Q \rightarrow \mathfrak{D}\left(Q_{q}\right)$. Let us write $\vec{q}: Q \rightarrow \mathfrak{D}\left(Q_{q}\right)$ for the resulting $\overrightarrow{\mathcal{D}}$-morphism.

The subclass of $Q \downarrow$ consisting of the elements $\vec{q}$ is directed, as the original projective filtration was directed, and hence saturates to a projective filtration. It is clear from its construction that this is functorial in $Q$.

\section{2. $\quad$ Reduced Filtrations}

Having looked at general filtrations, we now turn to a particular type of filtration. Let us consider the example of a topology on a set defined by a projective filtration. The structure of the category of sets means that we can ensure that each of the maps in the filtration is a surjection. This has certain advantages which we wish to mirror in our more general filtered categories. Although the definition below does 
not depend on any additional properties of the underlying category, in order to do anything useful with it we need to assume that this category is an extremally cowell-powered (extremal epi, mono) category. We also want to know that the forgetful functor $\overrightarrow{\mathcal{D}} \rightarrow \mathcal{D}$ has a right adjoint; for this we need to know that $\mathcal{D}$ has a terminal object.

Definition 3.11. Let $\mathcal{D}$ be a category. A projective filtration $Q$ on an object in $\mathcal{D}$ is said to be reduced if $Q$ has an initial subclass consisting of extremal epimorphisms.

We write $\overrightarrow{\mathcal{D}}$ for the full subcategory of $\overrightarrow{\mathcal{D}}$ consisting of reduced projective filtrations. Let $\mathfrak{S}: \overrightarrow{\mathcal{D}} \rightarrow \overrightarrow{\mathcal{D}}$ be the inclusion functor.

We could broaden our definition by first choosing a reasonable class of epimorphisms and considering those filtrations with morphisms in that class, but we shall only be interested in extremal epimorphisms and so we confine our attention to those.

Under the right conditions, the inclusion functor $\mathfrak{S}: \overrightarrow{\mathcal{D}} \rightarrow \overrightarrow{\mathcal{D}}$ has a right adjoint.

Proposition 3.12. Let $\mathcal{D}$ be an (extremal epi, mono) category. Then there is a reduction functor $\mathfrak{R}: \overrightarrow{\mathcal{D}} \rightarrow \overrightarrow{\mathcal{D}}$ which is faithful. The composition $\mathfrak{R S}: \overrightarrow{\mathcal{D}} \rightarrow \overrightarrow{\mathcal{D}}$ is the identity functor. The functor $\mathfrak{R}$ is right adjoint to $\mathfrak{S}$.

Proof. Let $Q$ be a projective filtration in $\mathcal{D}$. We define another projective filtration with the same underlying object in $\mathcal{D}$ as follows. Each element $q \in Q$ is a $\mathcal{D}$-morphism $q:|Q| \rightarrow Q_{q}$. By assumption on $\mathcal{D}$ this has a factorisation as $m_{q} \vec{q}$ where $\vec{q}$ is an extremal epimorphism and $m_{q}$ a monomorphism. Let us write $\vec{Q}_{q}$ for the target of ${ }^{-} \vec{q}$. We claim that the class of morphisms consisting of these $\vec{q}$ is directed. This follows from the diagonal property of an (extremal epi, mono) category. Suppose that $q_{1} \geqslant q_{2}$ in $Q$. Then there is some $\mathcal{D}$-morphism $f: Q_{q_{1}} \rightarrow Q_{q_{2}}$ such that $q_{2}=f q_{1}$. Thus the following is a commutative diagram in $\mathcal{D}$.

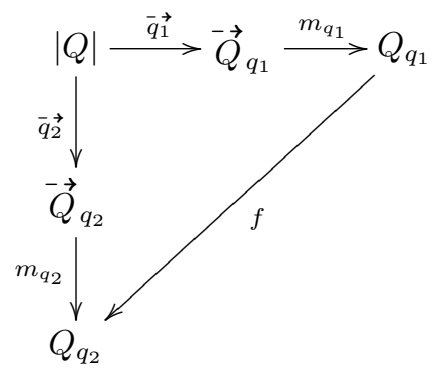

The diagonal property of the (extremal epi, mono)-factorisations now implies the existence of a $\mathcal{D}$-morphism $\vec{Q}_{q_{1}} \rightarrow \vec{Q}_{q_{2}}$ which fits into the above diagram. Hence in $\mid Q \|$ we have $\vec{q} \overrightarrow{1} \geqslant \overrightarrow{q_{2}}$. Thus as $Q$ is directed, the class $\{\vec{q}\}$ is also directed. Its saturation is thus a projective filtration which, by construction, is reduced. Let us write the result as $\vec{Q}$. We define $\mathfrak{R}$ on objects by $\mathfrak{R}(Q)=\vec{Q}$.

To define $\mathfrak{R}$ on morphisms we need to examine its interaction with pull backs. Let $Q$ be a projective filtration on an object in $\mathcal{D}$, let $D$ be an object in $\mathcal{D}$, and let 
$f: D \rightarrow|Q|$ be a $\mathcal{D}$-morphism. We wish to compare $f^{*}(\vec{Q})$ with $\bar{f}^{*}(\vec{Q})$. We obtain an initial subclass for $f^{*}(\vec{Q})$ by taking the extremal epimorphisms appearing in the (extremal epi, mono)-factorisations of elements of $Q$ and composing with $f$. That is, it consists of the $\mathcal{D}$-morphisms $\stackrel{-}{q} f: D \rightarrow \overrightarrow{Q_{q}}$ where $q:|Q| \rightarrow Q_{q}$ is an element of $Q$ with (extremal epi, mono)-factorisation $m_{q} \vec{q}$ and intervening object, $\vec{Q}_{q}$, in $\mathcal{D}$. On the other hand, an initial subclass of $\vec{f}^{*}(\vec{Q})$ consists of the extremal epimorphisms appearing in the (extremal epi, mono)-factorisations of the $\mathcal{D}$-morphisms $q f$ for $q$ in $Q$. For $q$ in $Q$ we therefore have the diagram

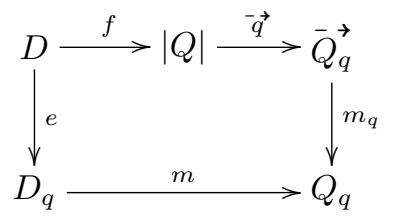

where $m e$ is the (extremal epi, mono)-factorisation of $q f$. As $\mathcal{D}$ is an (extremal epi, mono) category there is a $\mathcal{D}$-morphism $D_{q} \rightarrow \overrightarrow{Q_{q}}$ which fits into this diagram. Hence $f^{*}(\vec{Q}) \subseteq \vec{f}^{*}(\vec{Q})$

The proof that the defining subclass of $\vec{Q}$ is directed easily extends to show that if $Q_{1}$ and $Q_{2}$ are projective filtrations on the same underlying object in $\mathcal{D}$ with $Q_{1} \subseteq Q_{2}$ then $\overrightarrow{Q_{1}} \subseteq \overrightarrow{Q_{2}}$. Putting this together with the above, we see that if $f: Q_{1} \rightarrow Q_{2}$ is a $\overrightarrow{\mathcal{D}}$-morphism then $|f|^{*}\left(\overrightarrow{Q_{2}}\right) \subseteq|\vec{f}|^{*}\left(\overrightarrow{Q_{2}}\right) \subseteq \overrightarrow{Q_{1}}$ and so $|f|$ also underlies a $\overrightarrow{\mathcal{D}}$-morphism $\overrightarrow{Q_{1}} \rightarrow \overrightarrow{Q_{2}}$ which we denote by $\mathfrak{R}(f)$. As $f$ and $\mathfrak{R}(f)$ have the same underlying $\mathcal{D}$ morphism, this assignment clearly respects composition and identities whence we have a functor $\mathfrak{R}: \overrightarrow{\mathcal{D}} \rightarrow \overrightarrow{\mathcal{D}}$.

As (extremal epi, mono)-factorisations in $\mathcal{D}$ are unique up to canonical isomorphism, the composition $\overrightarrow{\mathcal{D}} \rightarrow \overrightarrow{\mathcal{D}} \rightarrow \overrightarrow{\mathcal{D}}$ is clearly the identity (saturation ensures that it is actually the identity, rather than just isomorphic to the identity). The obvious inclusion $Q \subseteq \vec{Q}$ provides the other natural transformation in the adjunction.

Since both forgetful functors $\overrightarrow{\mathcal{D}} \rightarrow \mathcal{D}$ and $\overrightarrow{\mathcal{D}} \rightarrow \mathcal{D}$ are faithful, and since the reduction functor $\overrightarrow{\mathcal{D}} \rightarrow \overrightarrow{\mathcal{D}}$ covers the identity on $\mathcal{D}$, the reduction functor must be faithful.

The discrete filtration functor, $\mathfrak{D}: \mathcal{D} \rightarrow \overrightarrow{\mathcal{D}}$, factors through $\overrightarrow{\mathcal{D}}$ with no modification since $\mathfrak{D}(D)$ contains the initial subclass $\{D \stackrel{1}{\rightarrow} D\}$ and every isomorphism is an extremal epimorphism. This provides us with a left adjoint to the forgetful functor $\overrightarrow{\mathcal{D}} \rightarrow \mathcal{D}$. The indiscrete filtration functor, $\mathfrak{I}: \mathcal{D} \rightarrow \overrightarrow{\mathcal{D}}$, (assuming that $\mathcal{D}$ has a terminal object) is not so fortunate. It is not even true that the terminal morphism $t_{D}: D \rightarrow T_{\mathcal{D}}$ is always an epimorphism. Thus we need to define $\overrightarrow{\mathfrak{I}}: \mathcal{D} \rightarrow \overrightarrow{\mathcal{D}}$ as the composition of $\mathfrak{I}$ with the reduction functor $\overrightarrow{\mathcal{D}} \rightarrow \overrightarrow{\mathcal{D}}$. This is right adjoint to the forgetful functor $\overrightarrow{\mathcal{D}} \rightarrow \mathcal{D}$. 


\subsection{Iso-Filtrations}

In this section we consider those filtered objects that are analogous to Hausdorff spaces and to complete uniform spaces. In order to work with these objects we need to make additional assumptions on our underlying category, namely that it be complete and extremally co-well-powered. As these filtered objects are a subclass of the reduced filtered objects we also retain the assumptions of the previous section. Thus we assume that $\mathcal{D}$ is a complete, extremally co-well-powered, (extremal epi, mono) category. Note that completeness implies the existence of a terminal object.

We start by defining another functor $\overrightarrow{\mathcal{D}} \rightarrow \mathcal{D}$ by taking the limit of a reduced filtration. We need to be in $\overrightarrow{\mathcal{D}}$ rather than $\overrightarrow{\mathcal{D}}$ to ensure that the definition makes sense.

Let us start with the most general definition. Let $\mathcal{D}$ be a category and $Q$ an object in $\overrightarrow{\mathcal{D}}$. We define a category $(Q, \mathcal{D})$ with objects elements of $Q$ and a morphism $q_{1} \rightarrow q_{2}$ is a $\mathcal{D}$-morphism, $d$, from the target of $q_{1}$ to the target of $q_{2}$ such that $d q_{1}=q_{2}$. An alternative description of this, which explains the notation, is as the full subcategory of the comma category $(|Q|, \mathcal{D})$ with class of objects the same as $Q$.

Note that the quasi-ordered class $Q$ when viewed as a category is a quotient of $(Q, \mathcal{D})$ under the relation $f \sim g$ if $f$ and $g$ have the same source and target.

There is an obvious functor $(Q, \mathcal{D}) \rightarrow \mathcal{D}$ which sends an object of $(Q, \mathcal{D})$ to its target, and which regards a morphism in $(Q, \mathcal{D})$ as a $\mathcal{D}$-morphism. Although the category $(Q, \mathcal{D})$ is usually large, the functor $(Q, \mathcal{D}) \rightarrow \mathcal{D}$ still might have a limit. By abuse of notation, we shall refer to the limit of the functor $(Q, \mathcal{D}) \rightarrow \mathcal{D}$ as the limit of $Q$ and write it as $\lim _{\longleftarrow} Q$.

When this limit exists, it is obvious that there is a natural $\mathcal{D}$-morphism

$$
|Q| \rightarrow \lim _{\longleftarrow} Q \text {. }
$$

The standard properties of limits show that if $f: Q_{1} \rightarrow Q_{2}$ is a $\overrightarrow{\mathcal{D}}$-morphism and $Q_{1}$ and $Q_{2}$ are such that both of the appropriate limits exist, then there is a corresponding $\mathcal{D}$-morphism $f^{\prime}: \lim Q_{1} \rightarrow \underset{\lim }{\longleftarrow} Q_{2}$ compatible with the above natural morphisms.

Definition 3.13. If $Q$ is an object in $\overrightarrow{\mathcal{D}}$ such that $\lim Q$ exists and the natural morphism $|Q| \rightarrow \lim Q$ is an isomorphism then we say that $Q$ is an iso-filtration on $|Q|$. We write $\overrightarrow{\mathcal{D}}$ for the full subcategory of $\overrightarrow{\mathcal{D}}$ consisting of all such objects in $\overrightarrow{\mathcal{D}}$. We write $\mathfrak{B}: \overrightarrow{\mathcal{D}} \rightarrow \overrightarrow{\mathcal{D}}$ for the inclusion functor.

Under reasonable conditions on $\mathcal{D}$ we can show that, in fact, every object in $\overrightarrow{\mathcal{D}}$ has a limit.

Proposition 3.14. If $\mathcal{D}$ is an extremally co-well-powered complete category, then every object in $\overrightarrow{\mathcal{D}}$ has a limit.

Proof. To prove the required result, we observe that if $Q$ is an object in $\overrightarrow{\mathcal{D}}$, it has an initial subclass consisting of extremal epimorphisms with source $|Q|$. As $\mathcal{D}$ is extremally co-well-powered, we can take this subclass to be small. It is, perforce, directed. We claim that this is an initial full subcategory of $(Q, \mathcal{D})$. Let $q_{1}$ and $q_{2}$ in 
$Q$ be extremal epimorphisms. Then as $q_{1}$ is an epimorphism, there can be at most one $\mathcal{D}$-morphism with the property that $d q_{1}=q_{2}$. Comparison of the ordering on elements of $Q$ with the definition of morphisms in $(Q, \mathcal{D})$ now shows that $(Q, \mathcal{D})\left(q_{1}, q_{2}\right)$ has at most one element and it has precisely one element if and only if $q_{1} \geqslant q_{2}$. Hence our initial subclass of $Q$ is a full subcategory of $(Q, \mathcal{D})$. It is clearly initial in $(Q, \mathcal{D})$.

As $\mathcal{D}$ is complete we can therefore find a limit of the functor $(Q, \mathcal{D}) \rightarrow \mathcal{D}$ by taking a limit of its restriction to our small initial subclass of $Q$. This is unique up to canonical isomorphism. It also depends functorially on $Q$ since if we have a $\overrightarrow{\mathcal{D}}$ morphism $Q_{1} \rightarrow Q_{2}$ then the pull back of $Q_{2}$ is contained in $Q_{1}$, whence we get a canonical $\mathcal{D}$-morphism on the limits, as they exist.

By the remarks preceding Definition 3.13, these limits fit together to form a functor $\overrightarrow{\mathcal{D}} \rightarrow \mathcal{D}$ and there is a natural transformation of functors $\overrightarrow{\mathcal{D}} \rightarrow \mathcal{D}$ from the forgetful functor to this limit functor.

Definition 3.15. We shall refer to the functor constructed above as the projective limit functor and write it as $\mathfrak{L}: \overrightarrow{\mathcal{D}} \rightarrow \mathcal{D}$.

Using the fact that a category of filtered objects is itself naturally filtered, we obtain the following important construction.

Proposition 3.16. Let $\mathcal{D}$ be a complete, extremally co-well-powered (extremal epi, mono) category. Then there is a natural lift of the projective limit functor $\mathfrak{L}: \overrightarrow{\mathcal{D}} \rightarrow$ $\mathcal{D}$ to a functor $\mathfrak{C}: \overrightarrow{\mathcal{D}} \rightarrow \overrightarrow{\mathcal{D}}$ which is left adjoint to the inclusion functor $\mathfrak{B}: \overrightarrow{\mathcal{D}} \rightarrow$ $\overrightarrow{\mathcal{D}}$. Moreover, the composition $\mathfrak{C} \mathfrak{B}: \overrightarrow{\mathcal{D}} \rightarrow \overrightarrow{\mathcal{D}}$ is naturally isomorphic to the identity functor on $\overrightarrow{\mathcal{D}}$.

Since the forgetful functor is naturally isomorphic to the projective limit functor when restricted to $\overrightarrow{\mathcal{D}}$ we do not need to specify which functor $\overrightarrow{\mathcal{D}} \rightarrow \mathcal{D}$ we are lifting along.

Proof. Let $Q$ be an object in $\overrightarrow{\mathcal{D}}$. Let $\iota:|Q| \rightarrow \mathfrak{L}(Q)$ be the canonical $\mathcal{D}$-morphism. Let $q$ be an element of $Q$. By the definition of a limit, there is a canonical $\mathcal{D}$-morphism

$$
\vec{q}: \mathfrak{L}(Q) \rightarrow Q_{q}
$$

such that $q=\vec{q} \iota$.

We claim that the subclass of $\mathfrak{L}(Q) \downarrow$ consisting of the morphisms $\vec{q}$ which arise in this fashion is a projective filtration. It is obviously non-empty and directed. To show that it is saturated, let $d: \mathfrak{L}(Q) \rightarrow D$ be a $\mathcal{D}$-morphism and suppose that it factors through $\vec{q}$ for some $q$ in $Q$, say $d=f \vec{q}$. Then $d \iota=f q$ whence $d \iota$ is in $Q$. The $\mathcal{D}$-morphism $f$ defines a morphism in $(Q, \mathcal{D})$ from $q$ to $d \iota$. Hence, by the definition of a limit, the canonical morphisms $\overrightarrow{(d \iota)}: \mathfrak{L}(Q) \rightarrow D$ and $\vec{q}: \mathfrak{L}(Q) \rightarrow Q_{q}$ satisfy $\overrightarrow{(d \iota)}=f \vec{q}$, whence $\overrightarrow{(d \iota)}=d$ and so $d$ is in our chosen subclass. Hence this subclass is saturated and we have a projective filtration.

This construction clearly depends functorially on $Q$.

Let us show that this is, in fact, a reduced projective filtration. We need to show that it has an initial subclass of extremal epimorphisms. It certainly has an initial 
subclass consisting of elements $\vec{q}$ where $q:|Q| \rightarrow Q_{q}$ is an extremal epimorphism. Since $q$ is an epimorphism, so is $\vec{q}$. Suppose that we have a factorisation $\vec{q}=m f$ where $m$ is a monomorphism; let $D$ be the intervening object in $\mathcal{D}$. As $\mathcal{D}$ is an (extremal epi, mono) category, the morphism $f \iota:|Q| \rightarrow D$ has an (extremal epi, mono)-factorisation, say $f \iota=m^{\prime} e^{\prime}$. Then $m m^{\prime} e^{\prime}=m f \iota=\vec{q} \iota=q$. As both $m$ and $m^{\prime}$ are monomorphisms, their composition is still a monomorphism whence as $q$ is an extremal epimorphism, $\mathrm{mm}^{\prime}$ is an isomorphism. The monomorphism $m$ is therefore a retraction, with right inverse $m^{\prime}\left(m m^{\prime}\right)^{-1}$, and so is an isomorphism. Hence $\vec{q}$ is an extremal epimorphism and so the projective filtration that we have defined on $\mathfrak{L}(Q)$ is reduced.

Let $Q^{\prime}$ denote this reduced projective filtration on $\mathfrak{L}(Q)$. We now claim that $Q^{\prime}$ is an iso-filtration. Consider the categories $(Q, \mathcal{D})$ and $\left(Q^{\prime}, \mathcal{D}\right)$. As $\left|Q^{\prime}\right|=\mathfrak{L}(Q)$ is the limit of $(Q, \mathcal{D}) \rightarrow \mathcal{D}$, the assignment $q \mapsto \vec{q}$ satisfies $\overrightarrow{(f q)}=f \vec{q}$ and thus we have a covariant functor $(Q, \mathcal{D}) \rightarrow\left(Q^{\prime}, \mathcal{D}\right)$ which on objects is $q \mapsto \vec{q}$ and which leaves morphisms alone (when viewed as $\mathcal{D}$-morphisms). The $\mathcal{D}$-morphism $\iota:|Q| \rightarrow \mathfrak{L}(Q)$ defines a covariant functor $\left(Q^{\prime}, \mathcal{D}\right) \rightarrow(Q, \mathcal{D})$. Since $\vec{q} \iota=q$, the composition is the identity functor on $(Q, \mathcal{D})$. The argument above which showed that the family $\{\vec{q}\}$ is saturated shows that the composition in the other direction is the identity functor on $\left(Q^{\prime}, \mathcal{D}\right)$. Since these functors do not change the targets of the objects when viewed as $\mathcal{D}$-morphisms and do not change the morphism sets it is clear that this isomorphism intertwines the two functors $(Q, \mathcal{D}) \rightarrow \mathcal{D}$ and $\left(Q^{\prime}, \mathcal{D}\right) \rightarrow \mathcal{D}$. Hence as $\mathfrak{L}(Q)$ is the limit of $(Q, \mathcal{D}) \rightarrow \mathcal{D}$ it is also the limit of $\left(Q^{\prime}, \mathcal{D}\right) \rightarrow \mathcal{D}$ and thus $Q^{\prime}$ is an iso-filtration.

We therefore have a functor $\mathfrak{C}: \overrightarrow{\mathcal{D}} \rightarrow \overrightarrow{\mathcal{D}}$ as required.

It is clear from this construction that if we start with an object in $\overrightarrow{\mathcal{D}}$ then all we do is replace the underlying object in $\mathcal{D}$ by an isomorphic one (with a specified isomorphism), whence the composition $\mathfrak{C} \mathfrak{B}$ is naturally isomorphic to the identity functor.

It is also clear from the construction that the natural transformation from the forgetful functor to $\mathfrak{L}$ underlies a natural transformation from the identity on $\overrightarrow{\mathcal{D}}$ to the composition $\mathfrak{B C}$.

It is obvious that these natural transformations produce the adjunction as stated.

Definition 3.17. We shall refer to the functor $\mathfrak{C}: \overrightarrow{\mathcal{D}} \rightarrow \overrightarrow{\mathcal{D}}$ as the filtered projective limit functor.

We have already noted that the discrete filtration functor, $\mathfrak{D}: \mathcal{D} \rightarrow \overrightarrow{\mathcal{D}}$, factors through $\overrightarrow{\mathcal{D}}$; it is equally easy to see that it factors through $\overrightarrow{\mathcal{D}}$. For comparison, $\overrightarrow{\mathfrak{I}}: \mathcal{D} \rightarrow \overrightarrow{\mathcal{D}}$ does not factor through $\overrightarrow{\mathcal{D}}$, and there is little point in considering the composition $\overrightarrow{\mathfrak{C}} \overrightarrow{\mathfrak{I}}$.

\subsection{Categorical Properties}

We wish to determine the categorical properties of the various categories of filtered objects. Certain results on $\overrightarrow{\mathcal{D}}, \overrightarrow{\mathcal{D}}$, and $\overrightarrow{\mathcal{D}}$ have depended on the categorical properties of $\mathcal{D}$. We shall want to work with categories such as $\stackrel{\overrightarrow{\mathcal{D}}}{\overrightarrow{\vec{a}}}$ and so we need to know whether 
the various properties of $\mathcal{D}$ lift to, say, $\overrightarrow{\mathcal{D}}$. We shall not consider categories such as

$\overrightarrow{\mathcal{D}}$ where the second type of filtration is more restrictive than the first. We also want to be able to apply the results of Section 2 and therefore we need to know other categorical properties to ensure that these apply.

In summary, we want to know the following.

1. Conditions on $\mathcal{D}$ to ensure that $\overrightarrow{\mathcal{D}}$ is an extremally co-well-powered (extremal epi, mono) category with a terminal object.

2. Conditions on $\mathcal{D}$ to ensure that $\overrightarrow{\mathcal{D}}$ is a complete extremally co-well-powered (extremal epi, mono) category.

3. Conditions on $\mathcal{D}$ to ensure that $\overrightarrow{\mathcal{D}}$ is co-complete.

4. How to form products and (finite) co-products in each of $\overrightarrow{\mathcal{D}}$ and $\overrightarrow{\mathcal{D}}$.

Let us state all the conditions on $\mathcal{D}$ that we need so that they are collected in one place. We assume that $\mathcal{D}$ is

1. complete,

2. co-complete,

3. an (extremal epi, mono) category, and

4. extremally co-well-powered.

Let us illustrate the various functors that we have. We denote the forgetful functor $\overrightarrow{\mathcal{D}} \rightarrow \mathcal{D}$ by $\mathfrak{U}$, though we shall still use the notation $|Q|$ for $\mathfrak{U}(Q)$. We shall also find it useful to have a notation for the forgetful functor $\overrightarrow{\mathcal{D}} \rightarrow \mathcal{D}$ so we denote this by $\mathfrak{Y}: \overrightarrow{\mathcal{D}} \rightarrow \mathcal{D}$. Let us write the discrete filtration functor $\mathfrak{D}$ as a functor into $\overrightarrow{\mathcal{D}}$ rather than $\overrightarrow{\mathcal{D}}$. We have the following (non-commuting!) diagram.

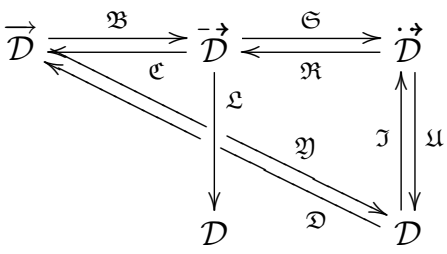

We have the following identities and adjunctions

$$
\begin{array}{rrrrl}
\mathfrak{R S} & =1, & \mathfrak{C} \mathfrak{B} \cong 1, & \mathfrak{Y} \mathfrak{D}=1, & \mathfrak{U} \mathfrak{I}=1, \\
\mathfrak{Y}=\mathfrak{U} \mathfrak{S} \mathfrak{B} & \mathfrak{U} \mathfrak{S} \mathfrak{R}=\mathfrak{U}, & \mathfrak{L} \mathfrak{B C} \cong \mathfrak{L}, & \mathfrak{D} \dashv \mathfrak{Y}, \\
\mathfrak{C} \dashv \mathfrak{B}, & \mathfrak{S} \dashv \mathfrak{R}, & \mathfrak{S} \mathfrak{B D} \dashv \mathfrak{U}, & \mathfrak{U} \dashv \mathfrak{I} .
\end{array}
$$

The functors $\mathfrak{B}, \mathfrak{S}, \mathfrak{D}$, and $\mathfrak{I}$ are fully faithful; $\mathfrak{U}$ is faithful, whence also $\mathfrak{R}$ and $\mathfrak{Y}$ are faithful. The category $\overrightarrow{\mathcal{D}}$ is a reflective subcategory of $\overrightarrow{\mathcal{D}}$.

We shall use some results from $[\mathbf{H S 7 3}, \S \mathrm{X}]$ to transfer results from $\overrightarrow{\mathcal{D}}$ to $\overrightarrow{\mathcal{D}}$. These results refer to reflective subcategories that are also full subcategories and closed under isomorphisms. These conditions are satisfied by $\overrightarrow{\mathcal{D}}$ inside $\overrightarrow{\mathcal{D}}$. 
We note in passing that in what follows we are essentially proving that $\overrightarrow{\mathcal{D}}$ is topological over $\mathcal{D}$. This is mildly reassuring since our intention was to model a specific type of topological space.

Let us start by defining push forward filtrations.

Lemma 3.18. Let $\left\{Q_{I}\right\}$ be a class of objects in $\overrightarrow{\mathcal{D}}$. Let $\left(D, f_{I}\right)$ be a sink for the underlying class of objects in $\mathcal{D}$. Then there is a reduced projective filtration, say $Q^{\prime}$, on $D$ such that

1. each $\mathcal{D}$-morphism $f_{i}:\left|Q_{i}\right| \rightarrow D$ lifts to a $\overrightarrow{\mathcal{D}}$-morphism $\overrightarrow{f_{i}}: Q_{i} \rightarrow Q^{\prime}$, and

2. if $Q^{\prime \prime}$ is an object in $\overrightarrow{\mathcal{D}}$ and $h: D \rightarrow\left|Q^{\prime \prime}\right|$ is a $\mathcal{D}$-morphism then $h$ lifts to a $\overrightarrow{\mathcal{D}}$-morphism $\vec{h}: Q^{\prime} \rightarrow Q^{\prime \prime}$ if and only if each $h f_{i}:\left|Q_{i}\right| \rightarrow\left|Q^{\prime \prime}\right|$ lifts to a $\overrightarrow{\mathcal{D}}$ morphism.

In the case that the class of objects in $\overrightarrow{\mathcal{D}}$ has only one element, say $Q$, and so the sink is just $f:|Q| \rightarrow D$ then we shall refer to the resulting object in $\overrightarrow{\mathcal{D}}$ as the push forward of $Q$ along $f$ and write it as $f_{*}(Q)$.

When the class of objects in $\overrightarrow{\mathcal{D}}$ is empty, clearly the discrete filtration has the required properties.

Proof. We define $Q^{\prime}$ as follows: it consists of all $\mathcal{D}$-morphisms, $g$, with source $D$ such that for each $i \in I, g f_{i}$ is in $Q_{i}$.

Firstly, $Q^{\prime}$ is not empty as $\mathcal{D}$ has a terminal object and so the terminal morphism from $D$ to this is in $Q^{\prime}$.

Secondly, $Q^{\prime}$ is directed. To see this, suppose that $g_{1}: D \rightarrow D_{1}$ and $g_{2}: D \rightarrow D_{2}$ are in $Q^{\prime}$. Consider the $\mathcal{D}$-morphism $\left(g_{1} \times g_{2}\right) \Delta: D \rightarrow D_{1} \times D_{2}$ (which exists as $\mathcal{D}$ is complete). If we can show that this lies in $Q^{\prime}$ then we are done as it precedes both $g_{1}$ and $g_{2}$. Let $i \in I$. As $Q_{i}$ is directed and saturated, $\left(g_{1} f_{i} \times g_{2} f_{i}\right) \Delta$ is in $Q_{i}$. By the functoriality of products, this is $\left(g_{1} \times g_{2}\right) \Delta f_{i}$. As this holds for all $i,\left(g_{1} \times g_{2}\right) \Delta$ is in $Q^{\prime}$.

Thirdly, $Q^{\prime}$ is saturated. To see this, suppose that $g_{1}$ is in $Q^{\prime}$ and $g_{2}=k g_{1}$. Then for $i \in I, g_{1} f_{i}$ is in $Q_{i}$ and so as this is saturated, $k g_{1} f_{i}$ is in $Q_{i}$. Hence $g_{2}$ is in $Q^{\prime}$.

We therefore have a projective filtration on $D$. We shall now show that it is reduced. Let $g: D \rightarrow D_{1}$ be in $Q^{\prime}$. As $\mathcal{D}$ is an (extremal epi, mono) category it has an (extremal epi, mono)-factorisation $g=m e$ with intervening object, $D_{2}$, in $\mathcal{D}$, say. Let $i \in I$. The $\mathcal{D}$-morphism $g f_{i}:\left|Q_{i}\right| \rightarrow D_{1}$ is in $Q_{i}$ and so since $Q_{i}$ is reduced it factors through an extremal epimorphism in $Q_{i}$, say $g f_{i}=h_{i} e_{i}$ with intervening object, $D_{3}$, in $\mathcal{D}$ and $e_{i}$ in $Q_{i}$. We therefore have a commutative diagram in $\mathcal{D}$.

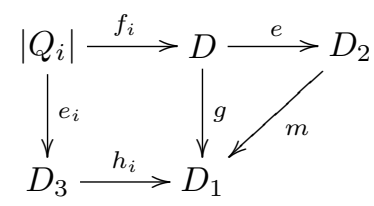

As $\mathcal{D}$ is an (extremal epi, mono) category, we can find a $\mathcal{D}$-morphism $D_{3} \rightarrow D_{2}$ which fits into this diagram. Hence $e f_{i}$ is in $Q_{i}$. Thus $e$ is in $Q^{\prime}$ which is now shown to be reduced. 
By construction the $\mathcal{D}$-morphisms $f_{i}:\left|Q_{i}\right| \rightarrow D$ lift to $\overrightarrow{\mathcal{D}}$-morphisms $Q_{i} \rightarrow Q^{\prime}$.

Let $h: D \rightarrow\left|Q^{\prime \prime}\right|$ be a $\mathcal{D}$-morphism. Let $q^{\prime \prime}$ be an element of $Q^{\prime \prime}$. Then $q^{\prime \prime} h$ is in $Q^{\prime}$ if and only if $q^{\prime \prime} h f_{i}$ is in $Q_{i}$ for all $i \in I$. Hence $h^{*}\left(Q^{\prime \prime}\right) \subseteq Q^{\prime}$ if and only if $\left(h f_{i}\right)^{*}\left(Q^{\prime \prime}\right) \subseteq Q_{i}$ for all $i \in I$.

We already have the notion of pull back filtrations in the not-necessarily-reduced case and it is easy to see that this generalises.

Lemma 3.19. Let $\left\{Q_{I}\right\}$ be a class of objects in $\overrightarrow{\mathcal{D}}$. Let $\left(D, f_{I}\right)$ be a source for the underlying class of objects in $\mathcal{D}$. Then there is a reduced projective filtration, say $Q^{\prime}$, on D such that

1. each $\mathcal{D}$-morphism $f_{i}: D \rightarrow\left|Q_{i}\right|$ lifts to a $\overrightarrow{\mathcal{D}}$-morphism $\overrightarrow{f_{i}}: Q^{\prime} \rightarrow Q_{i}$, and

2. if $Q^{\prime \prime}$ is an object in $\overrightarrow{\mathcal{D}}$ and $h:\left|Q^{\prime \prime}\right| \rightarrow D$ is a $\mathcal{D}$-morphism then $h$ lifts to a $\overrightarrow{\mathcal{D}}$-morphism $\vec{h}: Q^{\prime \prime} \rightarrow Q^{\prime}$ if and only if each $f_{i} h:\left|Q^{\prime \prime}\right| \rightarrow\left|Q_{i}\right|$ lifts to a $\overrightarrow{\mathcal{D}}$ morphism.

In the case that the class of objects in $\overrightarrow{\mathcal{D}}$ has one element we obtain the reduced pull back filtration, $\mathfrak{R}\left(f^{*}(Q)\right)$. If the class of objects in $\overrightarrow{\mathcal{D}}$ is empty, we obtain the reduced indiscrete filtration on $D$.

Proof. Each $\mathcal{D}$-morphism $f_{i}: D \rightarrow\left|Q_{i}\right|$ defines a pull back filtration $f_{i}^{*}\left(Q_{i}\right)$ on $D$. The union of these is a subclass of $\mathcal{D} \downarrow$. As $\mathcal{D}$ is complete, we can find a smallest projective filtration containing this subclass: first we include all finite products to ensure that it is directed and non-empty (via the empty product) and then we saturate it. Considered as an object in $\overrightarrow{\mathcal{D}}$, this clearly has the required properties.

We then apply $\mathfrak{R}$ to this object in $\overrightarrow{\mathcal{D}}$. The properties then follow from the fact that $\mathfrak{R}$ is right adjoint to the inclusion $\mathfrak{S}: \overrightarrow{\mathcal{D}} \rightarrow \overrightarrow{\mathcal{D}}$ and that both of these functors cover the identity on $\mathcal{D}$.

As the forgetful functor $\overrightarrow{\mathcal{D}} \rightarrow \mathcal{D}$ is faithful and has both a left and a right adjoint, it reflects and preserves monomorphisms and epimorphisms. Extremal epimorphisms are easy to characterise.

Corollary 3.20. $A \overrightarrow{\mathcal{D}}$-morphism $f: Q_{1} \rightarrow Q_{2}$ is an extremal epimorphism if and only if $|f|$ is an extremal epimorphism in $\mathcal{D}$ and $Q_{2}=|f|_{*}\left(Q_{1}\right)$.

Proof. Let us show the "only if" part first, so that we suppose that $f: Q_{1} \rightarrow Q_{2}$ is an extremal epimorphism. We need to show two things: that $|f|$ is an extremal epimorphism and that $Q_{2}=|f|_{*}\left(Q_{1}\right)$.

As the forgetful functor $\overrightarrow{\mathcal{D}} \rightarrow \mathcal{D}$ has a right adjoint, namely the reduced indiscrete functor, $|f|:\left|Q_{1}\right| \rightarrow\left|Q_{2}\right|$ is an epimorphism. Suppose that $|f|=m g$ with $m$ a monomorphism. Let $D$ be the intervening object in $\mathcal{D}$. We put the reduced pull back filtration on $D$ via $m$. Then by Lemma $3.19, m$ lifts to a $\overrightarrow{\mathcal{D}}$-morphism $\vec{m}: \mathfrak{R}\left(m^{*}\left(Q_{2}\right)\right)$ $\rightarrow Q_{2}$ and $g$ lifts to a $\overrightarrow{\mathcal{D}}$-morphism ${ }^{-} \vec{g}: Q_{1} \rightarrow \mathfrak{R}\left(m^{*}\left(Q_{2}\right)\right)$ with $\vec{m}^{-} \vec{g}=f$. As the forgetful functor is faithful, $\vec{m}$ is a monomorphism. Since $f$ is an extremal epimorphism, 
$\vec{m}$ is thus an isomorphism. Hence $m=|\vec{m}|$ is an isomorphism. Thus $|f|$ is an extremal epimorphism.

From Lemma 3.18, the identity on $\left|Q_{2}\right|$ underlies a $\overrightarrow{\mathcal{D}}$-morphism $|f|_{*}\left(Q_{1}\right) \rightarrow Q_{2}$ and so $f$ factorises as $Q_{1} \rightarrow|f|_{*}\left(Q_{1}\right) \rightarrow Q_{2}$. As the forgetful functor is faithful, the $\overrightarrow{\mathcal{D}}$-morphism $|f|_{*}\left(Q_{1}\right) \rightarrow Q_{2}$ is a monomorphism. Hence as $f$ is an extremal epimorphism, it is an isomorphism. As it covers the identity on $\left|Q_{2}\right|,|f|_{*}\left(Q_{1}\right)$ and $Q_{2}$ must in fact be the same projective filtrations.

Now let us show the "if" part. Let $f: Q_{1} \rightarrow Q_{2}$ be such that $|f|$ is an extremal epimorphism and $Q_{2}=f_{*}\left(Q_{1}\right)$. As the forgetful functor is faithful, $f$ is perforce an epimorphism. Suppose that we have a factorisation of $f$ as $m g$ with $m$ a monomorphism and intervening object, $Q^{\prime}$, in $\overrightarrow{\mathcal{D}}$. We therefore have a factorisation of $|f|$ as $|m||g|$. As the forgetful functor has a left adjoint, $|m|$ is a monomorphism. Hence, as $|f|$ is an extremal epimorphism, $|m|$ is an isomorphism. Consider the $\mathcal{D}$-morphism $|m|^{-1}|f|:\left|Q_{1}\right| \rightarrow\left|Q^{\prime}\right|$. This simplifies to $|g|$ which lifts to a $\overrightarrow{\mathcal{D}}$-morphism. Hence, by Lemma 3.18 since $Q_{2}=|f|_{*}\left(Q_{1}\right),|m|^{-1}$ lifts to a $\overrightarrow{\mathcal{D}}$-morphism and thus, as the forgetful functor is faithful, $m$ is an isomorphism. Hence $f$ is an extremal epimorphism.

This characterisation helps us prove the required extremality properties of $\overrightarrow{\mathcal{D}}$.

Corollary 3.21. $\overrightarrow{\mathcal{D}}$ is an (extremal epi, mono) category.

Proof. Let $f: Q_{1} \rightarrow Q_{2}$ be a $\overrightarrow{\mathcal{D}}$-morphism. The $\mathcal{D}$-morphism $|f|$ has an (extremal epi, mono)-factorisation, say $|f|=m e$. We can lift this to a factorisation of $f$ as $Q_{1} \rightarrow e_{*}\left(Q_{1}\right) \rightarrow Q_{2}$. This is an (extremal epi, mono) factorisation by Corollary 3.20 and as monomorphisms lift to monomorphisms.

To show uniqueness it is sufficient to show that we have the diagonal property. That is, suppose that we have a commutative square in $\overrightarrow{\mathcal{D}}$,

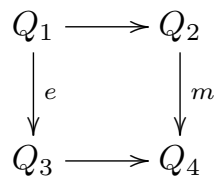

with $e$ an extremal epimorphism and $m$ a monomorphism. The underlying square in $\mathcal{D}$ has the same properties and thus there is a (unique) $\mathcal{D}$-morphism $h:\left|Q_{3}\right| \rightarrow\left|Q_{2}\right|$ which fits into the corresponding diagram in $\mathcal{D}$. Then he lifts to a $\overrightarrow{\mathcal{D}}$-morphism so as $Q_{3}=e_{*}\left(Q_{1}\right), h$ lifts to a $\overrightarrow{\mathcal{D}}$-morphism and thus $\overrightarrow{\mathcal{D}}$ has the (extremal epi, mono)diagonalisation property. Thus $\overrightarrow{\mathcal{D}}$ is an (extremal epi, mono)-category.

Corollary 3.22. $\overrightarrow{\mathcal{D}}$ is extremally co-well-powered.

Proof. Let $Q$ be an object in $\overrightarrow{\mathcal{D}}$. From the characterisation of extremal epimorphisms in $\overrightarrow{\mathcal{D}}$ we see that the forgetful functor $\overrightarrow{\mathcal{D}} \rightarrow \mathcal{D}$ defines a bijection from the class of isomorphism classes of extremal epimorphisms in $\overrightarrow{\mathcal{D}}$ with source $Q$ to the class of isomorphism classes of extremal epimorphisms in $\mathcal{D}$ with source $|Q|$. Hence the property of being extremally co-well-powered lifts from $\mathcal{D}$ to $\overrightarrow{\mathcal{D}}$. 


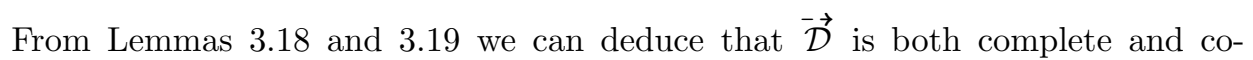
complete.

Proposition 3.23. $\overrightarrow{\mathcal{D}}$ is complete and co-complete.

Proof. This is a standard proof. We form limits and co-limits in $\overrightarrow{\mathcal{D}}$ by forming the limit or co-limit first in $\mathcal{D}$ and then putting the appropriate reduced filtration on the resulting object: the pull back filtration for the limit and the push forward for the co-limit.

We therefore have all our required properties of $\overrightarrow{\mathcal{D}}$. We now turn to $\overrightarrow{\mathcal{D}}$. Completeness and co-completeness follow directly from Proposition 3.23.

Corollary 3.24. $\overrightarrow{\mathcal{D}}$ is complete and co-complete.

Proof. It is a reflective, full subcategory of $\overrightarrow{\mathcal{D}}$ which is closed under isomorphism. Hence by $[\mathbf{H S 7 3}, 36.14,18]$, both completeness and co-completeness descend from $\overrightarrow{\mathcal{D}}$ to $\overrightarrow{\mathcal{D}}$.

Note that co-limits in $\overrightarrow{\mathcal{D}}$ are not simply the co-limits of the corresponding family in $\overrightarrow{\mathcal{D}}$ and therefore do not necessarily project down to the corresponding co-limit in $\mathcal{D}$. Rather we form the co-limit in $\overrightarrow{\mathcal{D}}$ and then apply the functor $\mathfrak{C}$ to the resulting object.

Extremal epimorphisms in $\overrightarrow{\mathcal{D}}$ are more complicated than in $\overrightarrow{\mathcal{D}}$ and so we need to work harder to prove that $\overrightarrow{\mathcal{D}}$ is an (extremal epi, mono) category and is extremally co-well-powered.

Proposition 3.25. $\overrightarrow{\mathcal{D}}$ is an (extremal epi, mono) category.

Proof. The proof of the factorisation property is an adaptation of the standard proof that every morphism in a complete well-powered category is (extremal epi, mono)factorisable.

Let $f: K_{1} \rightarrow K_{2}$ be a $\overrightarrow{\mathcal{D}}$-morphism. We consider the class of all factorisations $f=m h$ with $m$ a monomorphism. This is not empty as it contains the factorisation $(1, f)$. This is a quasi-ordered class with $\left(m_{1}, h_{1}\right) \geqslant\left(m_{2}, h_{2}\right)$ if there is a $\overrightarrow{\mathcal{D}}$-morphism from the source of $m_{1}$ to the source of $m_{2}$ making the obvious diagram commute. If this morphism exists, it is obviously unique and a monomorphism. There is an obvious functor from this quasi-ordered class to $\overrightarrow{\mathcal{D}}$.

We wish to show that this functor has a limit. We shall do this by showing that the class has a small initial subclass. Let $|f|=m_{D} e_{D}$ be the (extremal epi, mono)-factorisation of $|f|$ in $\mathcal{D}$ with intervening object, $D$, in $\mathcal{D}$. Let $f=m h$ be a factorisation of $f$ with $m$ a monomorphism and intervening object, $K$, in $\overrightarrow{\mathcal{D}}$. Then $|f|=|m||h|$ is a factorisation of $|f|$. As the forgetful functor $\overrightarrow{\mathcal{D}} \rightarrow \mathcal{D}$ has a left adjoint (the discrete filtration functor) it takes monomorphisms to monomorphisms and so $|m|$ is a 
monomorphism. Hence as $\mathcal{D}$ is an (extremal epi, mono) category there is a $\mathcal{D}$-morphism $g: D \rightarrow|K|$ making the following diagram commute.

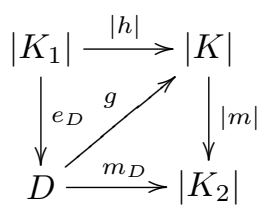

We pull back and reduce the projective filtration $K_{2}$ on $\left|K_{2}\right|$ via $m_{D}$ to one on $D$; let us write this as $Q$. The above diagram then lifts to $\overrightarrow{\mathcal{D}}$ with $Q$ in the lower left corner. Via the adjunction $\mathfrak{C} \dashv \mathfrak{B}$, the $\overrightarrow{\mathcal{D}}$-morphisms with source $Q$ factor through the natural morphism $Q \rightarrow \mathfrak{B C}(Q)$. We therefore have the diagram in $\overrightarrow{\mathcal{D}}$

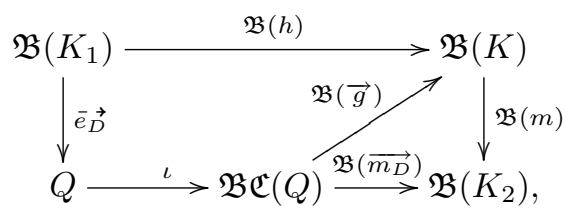

where $\iota$ is the canonical morphism.

We claim that $\vec{g}$ is a monomorphism. It is necessary and sufficient to show that $|\vec{g}|$ is a monomorphism as the forgetful functor is faithful and has a left adjoint. Thus let $d_{1}, d_{2}: D^{\prime} \rightarrow|\mathfrak{C}(Q)|$ be $\mathcal{D}$-morphisms such that $|\vec{g}| d_{1}=|\vec{g}| d_{2}$. As $|\mathfrak{C}(Q)|$ is the underlying object in $\mathcal{D}$ of an object in $\overrightarrow{\mathcal{D}}$, it is a limit and so $d_{1}$ and $d_{2}$ are completely determined by their compositions with the morphisms into the appropriate family. This family is the projective filtration $Q$ and for $q$ in $Q$ we have a $\mathcal{D}$-morphism $\tilde{q}:|\mathfrak{C}(Q)| \rightarrow Q_{q}$ such that $\tilde{q}|\iota|=q$.

The projective filtration $Q$ was defined as the reduction of the pull back of $K_{2}$ via $m_{D}$. It therefore has an initial family as follows: for each $k$ in $K_{2}$ the fact that $\mathcal{D}$ is an (extremal epi, mono) category implies the existence of a commutative diagram, unique up to canonical isomorphism,

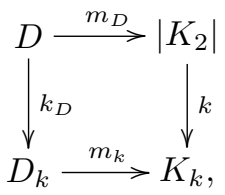

with $k_{D}$ an extremal epimorphism and $m_{k}$ a monomorphism. The family $k_{D}$ is initial for $Q$. We therefore have the following commutative diagram.

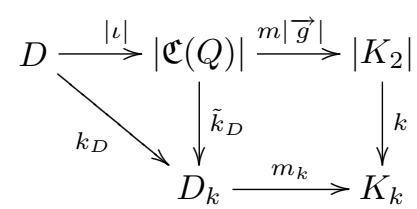

Thus $d_{1}$ and $d_{2}$ are completely determined by the compositions $\tilde{k}_{D} d_{i}$ for $k$ in $K_{2}$. Now 
as they satisfy $|\vec{g}| d_{1}=|\vec{g}| d_{2}$, for each $k$ in $K_{2}$ we have $k m|\vec{g}| d_{1}=k m|\vec{g}| d_{2}$ whence $m_{k} \tilde{k}_{D} d_{1}=m_{k} k_{D} d_{2}$. As $m_{k}$ is a monomorphism, we therefore have $\tilde{k}_{D} d_{1}=\tilde{k}_{D} d_{2}$ and thus $d_{1}=d_{2}$. Hence $|\vec{g}|$ is a monomorphism and thus so is $\vec{g}$.

The $\overrightarrow{\mathcal{D}}$-morphism $\overrightarrow{m_{D}}$ is equal to $m \vec{g}$ and so is a monomorphism. Let $\overrightarrow{e_{D}}: K_{1} \rightarrow$ $\mathfrak{C}(Q)$ be the $\overrightarrow{\mathcal{D}}$-morphism which, under the inclusion $\overrightarrow{\mathcal{D}} \subseteq \overrightarrow{\mathcal{D}}$, maps to $\iota \vec{e}_{D}$. The factorisation of $f$ in $\overrightarrow{\mathcal{D}}$ as $\overrightarrow{m_{D}} \overrightarrow{e_{D}}$ is thus in our class of factorisations and it precedes the factorisation $f=m h$.

The key property of this factorisation is that the morphism $\overrightarrow{e_{D}}$ is obtained by applying the functor $\mathfrak{C}$ to a $\overrightarrow{\mathcal{D}}$-morphism $\vec{e}_{D}: \mathfrak{B}\left(K_{1}\right) \rightarrow Q$ such that $\left|\overrightarrow{e_{D}}\right|=e_{D}$; note that $e_{D}$ depends only on $f$ and not on the factorisation that we were trying to dominate. This factorisation is therefore completely determined by the reduced projective filtration $Q$ on $D$.

As $\mathcal{D}$ is extremally co-well-powered, the class of all reduced projective filtrations on a specified object in $\mathcal{D}$ is actually a set. To see this, observe that a reduced projective filtration is completely determined by its subclass of extremal epimorphisms. Moreover, this subclass is closed under isomorphism and so is a union of equivalence classes of extremal epimorphisms, whence a reduced projective filtration is completely determined by an element of the power class of the class of equivalence classes of extremal epimorphisms emanating from the original object in $\mathcal{D}$. As $\mathcal{D}$ is extremally co-wellpowered, the class of equivalence classes of extremal epimorphisms is actually a set and so its power set is also a set. Hence the class of reduced projective filtrations on a given object in $\mathcal{D}$ is a set.

Thus our class of factorisations of the $\overrightarrow{\mathcal{D}}$-morphism, $f$, has an initial set and so, as $\overrightarrow{\mathcal{D}}$ is complete, has a limit. The proof that this limit is an (extremal epi, mono)factorisation of $f$ proceeds exactly as in the analogous proof for a morphism in a complete well-powered category. See, for example, [HS73, 17.8,17.16].

The proof that $\overrightarrow{\mathcal{D}}$ is in fact an (extremal epi, mono) category now follows since it is complete. See, for example, [HS73, 34.1].

It is worth pointing out that even if $\mathcal{D}$ were, in fact, a (regular epi, mono) category then it would not necessarily be true that $\overrightarrow{\mathcal{D}}$ was a (regular epi, mono) category.

Buried within the above proof are all the necessary pieces to prove the final property that we want.

Proposition 3.26. $\overrightarrow{\mathcal{D}}$ is extremally co-well-powered.

Proof. It is easy to see from the proof that $\overrightarrow{\mathcal{D}}$ is an (extremal epi, mono) category that every extremal epimorphism is obtained by applying $\mathfrak{C}$ to a $\overrightarrow{\mathcal{D}}$-morphism of the form $\mathfrak{B}(K) \rightarrow Q$ with underlying $\mathcal{D}$-morphism an extremal epimorphism (this is a necessary, but not sufficient, condition). To specify the isomorphism class of an extremal epimorphism in the category of iso-filtered objects of $\mathcal{D}$ it is therefore sufficient to specify the isomorphism class of the corresponding extremal epimorphism in $\mathcal{D}$ and a reduced projective filtration on the target.

Hence for an object, $K$, in $\overrightarrow{\mathcal{D}}$, the class of isomorphism classes of extremal epi- 
morphisms emanating from $K$ injects into

$$
\coprod_{\text {Iso(ex epi } d:|K| \rightarrow D)} \operatorname{Iso}\left(\overrightarrow{\mathcal{D}}_{D}\right)
$$

where Iso(ex epi $d:|K| \rightarrow D$ ) is the class of isomorphism classes of extremal epimorphisms with source $|K|$ and $\operatorname{Iso}\left(\overrightarrow{\mathcal{D}}_{D}\right)$ is the class of isomorphism classes of the fibre category of $\overrightarrow{\mathcal{D}} \rightarrow \mathcal{D}$ at $D$; that is, the class of reduced projective filtrations on $D$.

As $\mathcal{D}$ is extremally co-well-powered, all of the classes in this co-product are small. Hence the co-product is small and thus $\overrightarrow{\mathcal{D}}$ is extremally co-well-powered.

\subsection{The Canonical Filtration Functor}

In this section we shall construct a right adjoint to the forgetful functor $\mathfrak{Y}: \overrightarrow{\mathcal{D}} \rightarrow$ $\overrightarrow{\mathcal{D}}$. The indiscrete filtration functor, and its reduction, provide right adjoints to the forgetful functors $\overrightarrow{\mathcal{D}} \rightarrow \mathcal{D}$ and $\overrightarrow{\mathcal{D}} \rightarrow \mathcal{D}$ but in general the forgetful functor $\overrightarrow{\mathcal{D}} \rightarrow \mathcal{D}$ does not have a right adjoint. In the specific case $\overrightarrow{\overrightarrow{\mathcal{D}}} \rightarrow \overrightarrow{\mathcal{D}}$, however, we are able to construct one. It is a straightforward adaptation of the filtration functor for a filtered category as described in Example 3.10(2). We assume that $\mathcal{D}$ has the properties of Section 3.4.

It is simple to adapt the definition of Example 3.10(2) to define a functor $\overrightarrow{\mathcal{D}} \rightarrow \dot{\overrightarrow{\mathcal{D}}}$. For an object, $Q$, in $\overrightarrow{\mathcal{D}}$ the canonical filtration was defined by taking the projective filtration on $Q$ with initial subclass the family of $\overrightarrow{\mathcal{D}}$-morphisms $\stackrel{\vec{q}}{:} Q \rightarrow \mathfrak{S} \mathfrak{B D}\left(Q_{q}\right)$ (recall that we now regard $\mathfrak{D}$ as a functor into $\overrightarrow{\mathcal{D}}$ ). Similarly, we define a functor $\overrightarrow{\mathcal{D}} \rightarrow \overrightarrow{\overrightarrow{\mathcal{D}}}$ by taking the projective filtration on $K$ with initial subclass the family of $\overrightarrow{\mathcal{D}}$-morphisms $\vec{k}: K \rightarrow \mathfrak{D}\left(K_{k}\right)$.

Proposition 3.27. This functor factors through $\overrightarrow{\mathcal{D}}$.

Proof. We need to show first that the filtration defined above is reduced and then that it is an iso-filtration.

Let $K$ be an object in $\overrightarrow{\mathcal{D}}$ and let $P$ be the resulting object in $\overrightarrow{\overrightarrow{\mathcal{D}}}$. By construction, an initial subclass for the filtration $P$ is given by taking the $\overrightarrow{\mathcal{D}}$-morphisms

$$
\vec{k}: K \rightarrow \mathfrak{D}\left(K_{k}\right)
$$

for $k$ in $K$. It is obvious that we may refine this further and take $k$ in an initial subclass of $K$.

In particular, we can take those $k$ which are extremal epimorphisms. Let $k$ be one of these. We wish to show that $\vec{k}$ is an extremal epimorphism. Since $k$ is an epimorphism and the forgetful functor is faithful, $\vec{k}$ is an epimorphism. Now let $\vec{k}=m f$ be a factorisation with $m$ a monomorphism. Let $K^{\prime}$ be the intervening object in $\overrightarrow{\mathcal{D}}$. By applying the forgetful functor we obtain a factorisation of $k$ as $|m||f|$. The forgetful functor $\overrightarrow{\mathcal{D}} \rightarrow \mathcal{D}$ has a left adjoint, namely the discrete filtration functor, so preserves monomorphisms. Hence $|m|$ is a monomorphism and thus, as 
$k$ is an extremal epimorphism, $|m|$ is an isomorphism. Its inverse is a $\mathcal{D}$-morphism $K_{k} \rightarrow\left|K^{\prime}\right|$ and hence lifts to a $\overrightarrow{\mathcal{D}}$-morphism $\mathfrak{D}\left(K_{k}\right) \rightarrow K^{\prime}$. This lift is inverse to $m$ because the forgetful functor $\overrightarrow{\mathcal{D}} \rightarrow \mathcal{D}$ is faithful. Hence $\vec{k}$ is an extremal epimorphism and so $P$ is a reduced projective filtration.

To show that it is an iso-filtration we need to show that the limit of $P$ is isomorphic to $K$ via the canonical morphism. This follows from the description of limits in $\overrightarrow{\mathcal{D}}$ : they are formed by taking the underlying limit in $\mathcal{D}$ and putting the reduced pull back filtration on the resulting object in $\mathcal{D}$. In our case, the resulting object in $\mathcal{D}$ is (naturally isomorphic to) $|K|$ and it is obvious that the reduced pull back filtration of the family $|K| \rightarrow\left|\mathfrak{D}\left(K_{k}\right)\right|$ is again $K$.

Definition 3.28. We shall refer to the functor defined above as the canonical filtration functor and denote it by $\mathfrak{X}: \overrightarrow{\mathcal{D}} \rightarrow \overrightarrow{\overrightarrow{\mathcal{D}}}$.

Let us now show that this functor is the required adjoint.

Proposition 3.29. The canonical filtration functor $\mathfrak{X}: \overrightarrow{\mathcal{D}} \rightarrow \overrightarrow{\mathcal{D}}$ is right adjoint and right inverse to the forgetful functor $\mathfrak{Y}: \overrightarrow{\mathcal{D}} \rightarrow \overrightarrow{\mathcal{D}}$.

Proof. In this proof, $\mathfrak{Y}$ will refer exclusively to the forgetful functor $\overrightarrow{\mathcal{D}} \rightarrow \overrightarrow{\mathcal{D}}$ and we will use the notation $|-|$ for the forgetful functor $\overrightarrow{\mathcal{D}} \rightarrow \mathcal{D}$.

By construction, $\mathfrak{Y} \mathfrak{X}$ is the identity functor on $\overrightarrow{\mathcal{D}}$. This provides the natural transformation which will be the co-unit of the adjunction.

Let $L$ be an object in $\overrightarrow{\mathcal{D}}$. Both $L$ and $\mathfrak{X Y}(L)$ are iso-filtrations on the same underlying object in $\overrightarrow{\mathcal{D}}$, namely $\mathfrak{Y}(L)$. We shall show that $\mathfrak{X Y}(L) \subseteq L$ as projective filtrations on $\mathfrak{Y}(L)$. By construction, $\mathfrak{X Y}(L)$ is the projective filtration on $\mathfrak{Y}(L)$ with initial subclass

$$
\vec{k}: \mathfrak{Y}(L) \rightarrow \mathfrak{D}\left(\mathfrak{Y}(L)_{k}\right)
$$

for $k$ in $\mathfrak{Y}(L)$. Now $L$ is an iso-filtration on $\mathfrak{Y}(L)$ and so the canonical morphism

$$
\mathfrak{Y}(L) \rightarrow \underset{l}{\lim _{l}} L_{l}
$$

is an isomorphism of objects in $\overrightarrow{\mathcal{D}}$, where the limit is over $l$ in $L$. Limits in $\overrightarrow{\mathcal{D}}$ are formed by taking the corresponding limit in $\mathcal{D}$ and then putting the reduced pull back filtration on the resulting object. Thus an initial subclass of $\mathfrak{Y}(L)$ consists of the extremal epimorphisms coming from the (extremal epi, mono)-factorisation of $\mathcal{D}$-morphisms of the form

$$
|\mathfrak{Y}(L)| \stackrel{|l|}{\longrightarrow}\left|L_{l}\right| \stackrel{l^{\prime}}{\rightarrow} L_{l, l^{\prime}}
$$

for $l$ in $L$ and $l^{\prime}$ in $L_{l}$. We can assume that $l$ and $l^{\prime}$ are themselves in initial subclasses of their respective filtrations and so we can assume that they are extremal epimorphisms in their respective categories.

Let us show that the composition $l^{\prime}|l|$ is itself an extremal epimorphism. It is an epimorphism because $l^{\prime}$ and $|l|$ are both epimorphisms. Let $l^{\prime}|l|=m e$ be the 
(extremal epi, mono)-factorisation in $\mathcal{D}$ of $l^{\prime}|l|$ with intervening object, $D$, in $\mathcal{D}$. By the above, $e$ is in $\mathfrak{Y}(L)$ and thus lifts to a $\overrightarrow{\mathcal{D}}$-morphism $\tilde{e}: \mathfrak{Y}(L) \rightarrow \mathfrak{D}(D)$. The $\mathcal{D}$-morphism $m: D \rightarrow L_{l, l^{\prime}}$ lifts to a $\overrightarrow{\mathcal{D}}$-morphism $\mathfrak{D}(m): \mathfrak{D}(D) \rightarrow \mathfrak{D}\left(L_{l, l^{\prime}}\right)$. This is again a monomorphism as the forgetful functor is faithful. Since $l^{\prime}$ is in $L_{l}$ it also lifts to a $\overrightarrow{\mathcal{D}}$-morphism $\tilde{l^{\prime}}: L_{l} \rightarrow \mathfrak{D}\left(l_{l, l^{\prime}}\right)$. As lifts of $\mathcal{D}$-morphisms to $\overrightarrow{\mathcal{D}}$-morphisms are unique, we therefore have the following commutative diagram in $\overrightarrow{\mathcal{D}}$.

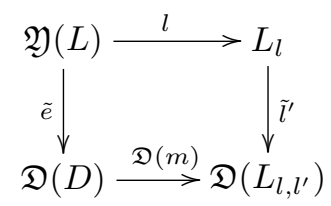

Since $\overrightarrow{\mathcal{D}}$ is an (extremal epi, mono) category there is a $\overrightarrow{\mathcal{D}}$-morphism $g: L_{l} \rightarrow \mathfrak{D}(D)$ which fits into the above diagram. Applying the forgetful functor we see that $l^{\prime}=m|g|$. As $l^{\prime}$ is an extremal epimorphism we see that $m$ is an isomorphism. Hence $l^{\prime}|l|$ is isomorphic to $e$ and thus is an extremal epimorphism.

Thus an initial subclass for $\mathfrak{Y}(L)$ is the family of $\mathcal{D}$-morphisms

$$
|\mathfrak{Y}(L)| \stackrel{|l|}{\longrightarrow}\left|L_{l}\right| \stackrel{l^{\prime}}{\rightarrow} L_{l, l^{\prime}}
$$

with $l$ in $L$ and $l^{\prime}$ in $L_{l}$. Thus an initial subclass of $\mathfrak{X Y}(L)$ consists of the family of $\overrightarrow{\mathcal{D}}$-morphisms

$$
|L| \stackrel{l}{\rightarrow} L_{l} \stackrel{\tilde{l}^{\prime}}{\rightarrow} \mathfrak{D}\left(L_{l, l^{\prime}}\right) .
$$

As each of these factors through $l$ it is in $L$. Hence $\mathfrak{X Y}(L) \subseteq L$ and so the identity $\overrightarrow{\mathcal{D}}$-morphism on $\mathfrak{Y}(L)$ lifts to a $\overrightarrow{\mathcal{D}}$-morphism $L \rightarrow \mathfrak{X Y Y}(L)$.

These lifts fit together to define a natural transformation of functors from the identity on $\overrightarrow{\mathcal{D}}$ to $\mathfrak{X Y}$ : all the necessary diagrams commute because they do in $\overrightarrow{\mathcal{D}}$. This will be the unit of our adjunction.

Our functors, $\mathfrak{Y}$ and $\mathfrak{X}$, are both lifts of the identity functor on $\overrightarrow{\mathcal{D}}$ along the forgetful functor $\overrightarrow{\mathcal{D}} \rightarrow \overrightarrow{\mathcal{D}}$, one lifting the source and the other the target. The natural transformations, $\mathfrak{Y} \mathfrak{X} \rightarrow I$ and $I \rightarrow \mathfrak{X Y}$, are both lifts of the identity natural transformation $I \rightarrow I$ on $\overrightarrow{\mathcal{D}}$. Therefore for an object, $L$, in $\overrightarrow{\overrightarrow{\mathcal{D}}}$ and object, $K$, in $\overrightarrow{\mathcal{D}}$ the forgetful functor $\mathfrak{Y}: \overrightarrow{\overrightarrow{\mathcal{D}}} \rightarrow \overrightarrow{\mathcal{D}}$ induces a commutative diagram of morphisms of hom-sets.

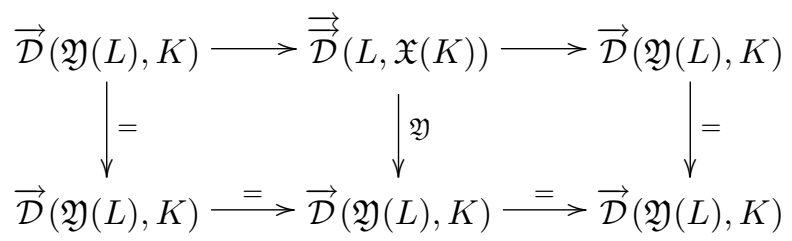

As the forgetful functor is faithful, the morphisms in the upper line are isomorphisms and hence $\mathfrak{X}$ is right adjoint to $\mathfrak{Y}$. 


\subsection{Lifts of Functors}

In this section we shall examine certain lifts of functors involving filtered categories. The two lifts that we shall consider are described in the next definition. Recall that for a functor $\mathfrak{G}: \mathcal{D} \rightarrow \mathcal{E}$ we defined, in Section 3.1, a corresponding functor $\overrightarrow{\mathfrak{G}}: \overrightarrow{\mathcal{D}} \rightarrow \stackrel{\overrightarrow{\mathcal{E}}}{ }$.

The conditions that we impose on our categories in the following theorems are not minimal. Recall that we write $\mathfrak{Y}: \overrightarrow{\mathcal{D}} \rightarrow \overrightarrow{\mathcal{D}}$ for the forgetful functor.

Definition 3.30. Let $\mathcal{D}$ and $\mathcal{E}$ be complete, co-complete, extremally co-well-powered, (extremal epi, mono) categories. Let $\mathfrak{G}: \overrightarrow{\mathcal{D}} \rightarrow \mathcal{E}$ be a covariant functor. We define $\overrightarrow{\mathfrak{G}}: \overrightarrow{\mathcal{D}} \rightarrow \overrightarrow{\mathcal{E}}$ by

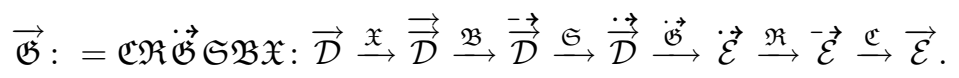

Let $\mathfrak{H}: \mathcal{E} \rightarrow \overrightarrow{\mathcal{D}}$ be a covariant functor. We define $\overrightarrow{\mathfrak{H}}: \overrightarrow{\mathcal{E}} \rightarrow \overrightarrow{\mathcal{D}}$ by

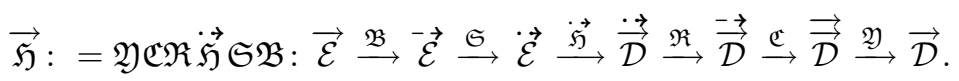

We trust that there will be no confusion with using the same notation for two different constructions. Note that in these definitions we have two different instances of various functors. In the definition of $\overrightarrow{\mathfrak{G}}$ the inclusion functor $\mathfrak{B}$ is from $\overrightarrow{\mathcal{D}}$ to $\overrightarrow{\mathcal{D}}$ whereas in the definition of $\overrightarrow{\mathfrak{H}}$ it is from $\overrightarrow{\mathcal{E}}$ to $\overrightarrow{\mathcal{E}}$. We trust that this also will not cause confusion.

We wish to prove two results about these constructions. The first gives a condition whereby the first construction is associative. The second relates to adjunctions.

Theorem 3.31. Let $\mathcal{D}, \mathcal{E}$, and $\mathcal{F}$ be complete, co-complete, extremally co-well-powered, (extremal epi, mono) categories. Let $\mathfrak{G}: \overrightarrow{\mathcal{D}} \rightarrow \mathcal{E}$ and $\mathfrak{H}: \overrightarrow{\mathcal{E}} \rightarrow \mathcal{F}$ be covariant functors. Then if $\mathfrak{H}$ preserves monomorphisms, there is a natural isomorphism of functors $\overrightarrow{\mathcal{D}} \rightarrow \overrightarrow{\mathcal{F}}$

$$
\overrightarrow{\mathfrak{H} \vec{G}} \cong \overrightarrow{\mathfrak{H}} \overrightarrow{\mathfrak{G}}
$$

satisfying the obvious coherence for triples.

Proof. Let us expand out the two sides to make clear what we have to prove.

$$
\begin{aligned}
& \overrightarrow{\mathfrak{H} \mathfrak{G}}=\mathfrak{C} \mathfrak{R} \vec{H} \overrightarrow{\mathfrak{G}} \mathfrak{S} \mathfrak{B X}=\mathfrak{C} \mathfrak{R} \overrightarrow{\mathfrak{H}} \overrightarrow{\vec{G}} \mathfrak{S} \mathfrak{B X}, \\
& \overrightarrow{\mathfrak{H}} \overrightarrow{\mathfrak{G}}=\mathfrak{C} \mathfrak{R} \overrightarrow{\mathfrak{H}} \mathfrak{S} \mathfrak{B X C} \mathfrak{R} \overrightarrow{\mathfrak{G}} \mathfrak{S} \mathfrak{B X}=\mathfrak{C} \mathfrak{R} \overrightarrow{\mathfrak{H}} \mathfrak{S} \mathfrak{B X} \overrightarrow{\mathfrak{G}} .
\end{aligned}
$$

From this it is clear that the first step is to compare $\overrightarrow{\mathfrak{G}} \mathfrak{S} \mathfrak{B} \mathfrak{X}$ with $\mathfrak{S} \mathfrak{B} \mathfrak{X} \overrightarrow{\mathfrak{G}}$. These are functors $\overrightarrow{\mathcal{D}} \rightarrow \overrightarrow{\overrightarrow{\mathcal{E}}}$. Using the fact that $|\overrightarrow{\overrightarrow{\mathfrak{G}}}(-)|=\overrightarrow{\mathfrak{G}}(|-|)$ we see that both $\overrightarrow{\mathfrak{G}} \mathfrak{S} \mathfrak{B X}$ and $\mathfrak{S} \mathfrak{B} \mathfrak{X} \overrightarrow{\mathfrak{G}}$ are lifts of $\overrightarrow{\mathfrak{G}}$ along the forgetful functor $\overrightarrow{\overrightarrow{\mathcal{E}}} \rightarrow \overrightarrow{\mathcal{E}}$. To compare these lifts we need to examine the resulting filtrations on $\overrightarrow{\mathfrak{G}}(K)$ for an object, $K$, in $\overrightarrow{\mathcal{D}}$.

Firstly, let us establish some notation. Applying $\mathfrak{R}$ to an object, $Q$, in $\overrightarrow{\mathcal{D}}$ does not change the underlying object in $\mathcal{D}$, it merely alters the filtration. An initial class for $\mathfrak{R}(Q)$ is given by taking the extremal epimorphisms which come from the (extremal 
epi, mono)-factorisations of the $\mathcal{D}$-morphisms in $Q$. As before, for $q:|Q| \rightarrow Q_{q}$ in $Q$ let us write

$$
\vec{q}:|Q| \rightarrow \overrightarrow{Q_{q}}
$$

for the corresponding extremal epimorphism.

Let $K$ be an object in $\overrightarrow{\mathcal{D}}$. Let us examine $\overrightarrow{\mathfrak{G}}(K)$. An initial subclass of the filtration $\mathfrak{X}(K)$ is given by the family

$$
K \rightarrow \mathfrak{D}\left(K_{k}\right)
$$

for $k$ in $K$. Here and henceforth we will suppress the label for the morphism as the notation rapidly becomes unwieldy; in each case it will be the obvious morphism derived from $k$.

An initial subclass of the filtration $\overrightarrow{\mathfrak{G}} \mathfrak{S} \mathfrak{B} \mathfrak{X}(K)$ is thus given by the family

$$
\mathfrak{G}(K) \rightarrow \mathfrak{G} \mathfrak{D}\left(K_{k}\right)
$$

and of $\mathfrak{R} \overrightarrow{\mathfrak{G}} \mathfrak{S} \mathfrak{B X}(K)$ by

$$
\mathfrak{G}(K) \rightarrow \overline{\mathfrak{G}} \overline{\mathfrak{D}}\left(\bar{K}_{k}\right)
$$

To get $\overrightarrow{\mathfrak{G}}(K)$ we apply $\mathfrak{C}$ which replaces the source of these morphisms by the appropriate limit. This produces an initial family for $\overrightarrow{\mathfrak{G}}(K)$ consisting of the $\overrightarrow{\overrightarrow{\mathcal{E}}}$-morphisms

$$
|\overrightarrow{\mathfrak{G}}(K)| \rightarrow \overline{\mathfrak{G}} \overline{\mathfrak{D}}\left(\bar{K}_{k}\right)
$$

We can read off from this an initial family for $\mathfrak{S} \mathfrak{B} \mathfrak{\mathfrak { G }}(K)$. It consists of the $\overrightarrow{\mathcal{E}}$ morphisms

$$
\overrightarrow{\mathfrak{G}}(K) \rightarrow \mathfrak{D}\left(\overline{\mathfrak{G}} \overline{\mathfrak{D}}\left(\bar{K}_{k}\right)\right)
$$

By applying the above as far as (1) with $\overrightarrow{\mathfrak{G}}$ in place of $\mathfrak{G}$ we can also read off an initial family for $\overrightarrow{\mathfrak{G}} \mathfrak{S} \mathfrak{B} \mathfrak{X}(K)$. It consists of the $\overrightarrow{\mathcal{E}}$-morphisms

$$
\overrightarrow{\mathfrak{G}}(K) \rightarrow \overrightarrow{\mathfrak{G}} \mathfrak{D}\left(K_{k}\right) .
$$

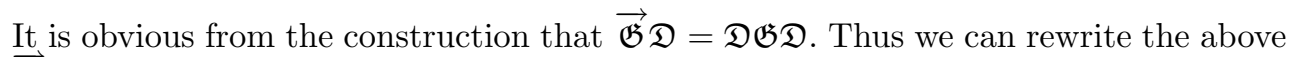
$\overrightarrow{\mathcal{E}}$-morphisms as

$$
\overrightarrow{\mathfrak{G}}(K) \rightarrow \mathfrak{D} \mathfrak{G} \mathfrak{D}\left(K_{k}\right)
$$

The objects, $\overline{\mathfrak{G}} \overline{\mathfrak{D}}\left(\bar{K}_{k}\right)$, in $\mathcal{E}$ are defined (up to canonical isomorphism) by the (extremal epi, mono)-factorisations

$$
\mathfrak{G}(K) \rightarrow \overline{\mathfrak{G}} \overline{\mathfrak{D}}\left(\bar{K}_{k} \overrightarrow{)} \rightarrow \mathfrak{G} \mathfrak{D}\left(K_{k}\right)\right.
$$

From the construction of $\overrightarrow{\mathfrak{G}}$ as a limit (via the functor $\mathfrak{C}$ ) we see that we can replace $\mathfrak{G}(K)$ by $|\overrightarrow{\mathfrak{G}}(K)|$ in this. Thus, as projective filtrations on $\overrightarrow{\mathfrak{G}}(K), \overrightarrow{\mathfrak{G}} \mathfrak{S} \mathfrak{B} \mathfrak{X}(K)$ is contained in $\mathfrak{S} \mathfrak{B} \mathfrak{X} \vec{G}(K)$. From this we deduce that the identity on $\overrightarrow{\mathfrak{G}}(K)$ lifts to a 
$\overrightarrow{\overrightarrow{\mathcal{E}}}$-morphism

$$
\mathfrak{S B \mathfrak { X }} \overrightarrow{\mathfrak{G}}(K) \rightarrow \overrightarrow{\overrightarrow{\mathfrak{G}}} \mathfrak{S} \mathfrak{B X}(K)
$$

As this is a lift of the identity on $\overrightarrow{\mathfrak{G}}$ it defines a natural transformation $\mathfrak{S} \mathfrak{B} \mathfrak{X} \vec{G} \rightarrow$ $\overrightarrow{\vec{G}} \mathfrak{S} \mathfrak{B X}$.

We claim that this becomes a natural isomorphism after reduction. To prove this claim, consider the following diagrams in $\overrightarrow{\mathcal{E}}$.
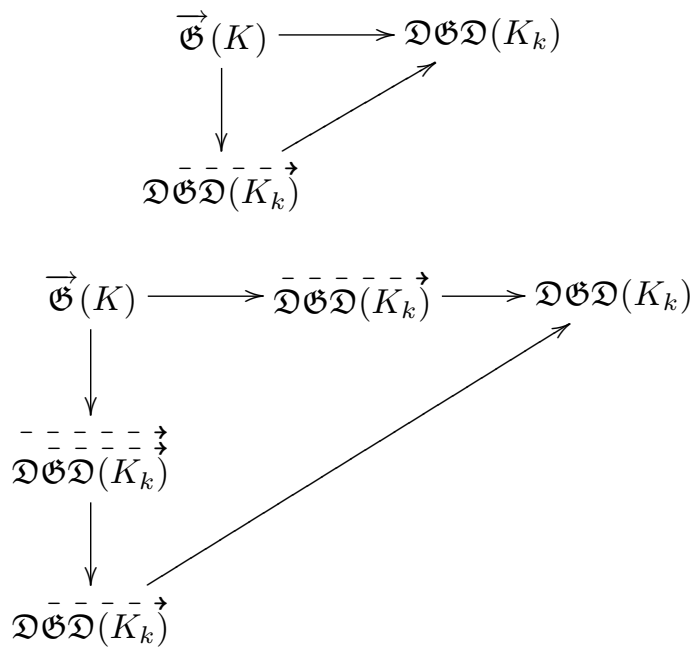

The second is derived from the first by taking the (extremal epi, mono)-factorisations of the vertical and horizontal morphisms. The diagonal arrow in each diagram is a monomorphism since it is $\mathfrak{D}$ applied to a monomorphism and $\mathfrak{D}$ preserves monomorphisms. Thus by the diagonalisation property of an (extremal epi, mono) category, there is an isomorphism

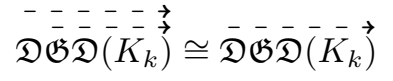

fitting in to the second diagram.

Thus the natural transformation

$$
\mathfrak{S} \mathfrak{B X} \overrightarrow{\mathfrak{G}} \rightarrow \overrightarrow{\overrightarrow{\mathfrak{G}}} \mathfrak{S} \mathfrak{B X}
$$

induces a natural isomorphism

$$
\mathfrak{R S B X} \overrightarrow{\mathfrak{G}} \rightarrow \mathfrak{\vec { R } \mathfrak { G }} \mathfrak{S B X} .
$$

This is still not quite what is needed as there is an occurrence of the functor $\mathfrak{R}$ which is not present in the expansions of the two functors that we wish to compare. What we shall now see is that we could easily insert $\mathfrak{R}$ at the appropriate juncture without changing the result; actually we insert $\mathfrak{S} \Re$.

There is a natural transformation $\mathfrak{S} \mathfrak{R} \rightarrow 1$ coming from the adjunction $\mathfrak{S} \dashv \mathfrak{R}$. Applying $\mathfrak{R} \dot{\mathfrak{H}}$ yields a natural transformation $\mathfrak{R} \dot{\mathfrak{H}} \mathfrak{S} \mathfrak{R} \rightarrow \mathfrak{R} \dot{\mathfrak{H}}$. We wish to show that 
this is a natural isomorphism. Let $Y$ be an object in $\overrightarrow{\overrightarrow{\mathcal{E}}}$. Using notation as above, we have the following initial classes of the projective filtrations

$$
\begin{aligned}
& \left(\mathfrak{H}(|Y|) \rightarrow \overline{\mathfrak{H}}\left(\overline{Y_{y}}\right) \text { for } \quad \mathfrak{R} \overrightarrow{\mathfrak{H}}(Y),\right. \\
& \left(\mathfrak{H}(|Y|) \rightarrow \overrightarrow{\mathfrak{H}}\left(\overrightarrow{Y_{y}}\right)\right) \text { for } \quad \mathfrak{R} \overrightarrow{\mathfrak{H}} \mathfrak{S} \mathfrak{R}(Y)
\end{aligned}
$$

and the natural transformation is the identity on $\mathfrak{H}(|Y|)$. This natural transformation comes from the fact that the diagonalisation property of an (extremal epi, mono) category allows us to add in the required morphism on the inner diagonal of this diagram:

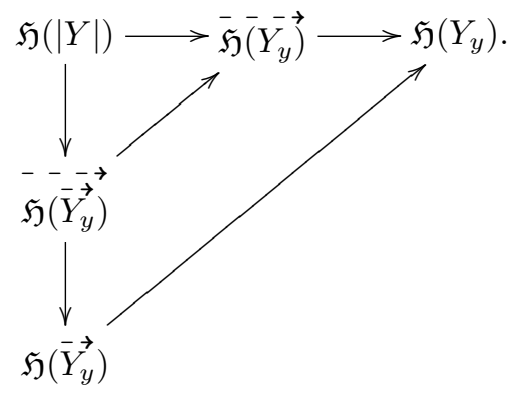

By assumption, $\mathfrak{H}$ preserves monomorphisms and thus the outer diagonal is a monomorphism. Using the diagonalisation property again, the morphism on the inner diagonal is therefore an isomorphism. Thus the identity on $\mathfrak{H}(|Y|)$ underlies an $\overrightarrow{\mathcal{F}}$-isomorphism $\mathfrak{R} \overrightarrow{\mathfrak{H}} \mathfrak{S} \mathfrak{R}(Y) \rightarrow \mathfrak{R} \dot{\mathfrak{H}}(Y)$ and we therefore have the required natural isomorphism.

Hence we have natural isomorphisms

$$
\begin{aligned}
\overrightarrow{\mathfrak{H} \vec{G}} & =\mathfrak{C} \mathfrak{R} \overrightarrow{\mathfrak{H}} \overrightarrow{\mathfrak{G}} \mathfrak{S} \mathfrak{B X} \\
& \cong \mathfrak{C} \mathfrak{R} \overrightarrow{\mathfrak{H}} \mathfrak{S} \mathfrak{R} \overrightarrow{\mathfrak{G}} \mathfrak{S} \mathfrak{B X} \\
& \cong \mathfrak{C} \mathfrak{R} \ddot{\mathfrak{H}} \mathfrak{S} \mathfrak{R S} \mathfrak{B} \mathfrak{X} \overrightarrow{\mathfrak{G}} \\
& =\mathfrak{C} \mathfrak{R} \overrightarrow{\mathfrak{H}} \mathfrak{S} \mathfrak{B} \mathfrak{X} \overrightarrow{\mathfrak{G}} \\
& =\overrightarrow{\mathfrak{H}} \overrightarrow{\mathfrak{G}}
\end{aligned}
$$

as required.

That this satisfies the required coherence axiom is a simple deduction from the fact that the natural transformations involved in the above all derive from lifting identity morphisms along faithful functors. Thus one can faithfully map the required diagram down to one which obviously commutes.

The constructions of Definition 3.30 extend in the obvious way to morphisms (that is, natural transformations). Thus the first construction gives a functor from the category of covariant functors from $\overrightarrow{\mathcal{D}}$ to $\mathcal{E}$ to the category of covariant functors from $\overrightarrow{\mathcal{D}}$ to $\overrightarrow{\mathcal{E}}$, which on objects is given by $\mathfrak{G} \mapsto \overrightarrow{\mathfrak{G}}$. Similarly, the second construction 
gives a functor from the category of covariant functors from $\mathcal{E}$ to $\overrightarrow{\mathcal{D}}$ to the category of covariant functors from $\overrightarrow{\mathcal{E}}$ to $\overrightarrow{\mathcal{D}}$, which on objects is given by $\mathfrak{H} \mapsto \overrightarrow{\mathfrak{H}}$.

The rest of this section in concerned with proving the following result on adjunctions.

Theorem 3.32. Let $\mathcal{D}$ and $\mathcal{E}$ be complete, co-complete, extremally co-well-powered, (extremal epi, mono) categories. Let $\mathfrak{G}: \overrightarrow{\mathcal{D}} \rightarrow \mathcal{E}$ and $\mathfrak{H}: \mathcal{E} \rightarrow \overrightarrow{\mathcal{D}}$ be covariant functors such that $\mathfrak{H}$ is left adjoint to $\mathfrak{G}$. If $\mathfrak{H}$ preserves extremal epimorphisms then $\overrightarrow{\mathfrak{H}}$ is left adjoint to $\overrightarrow{\mathfrak{G}}$.

We shall prove this theorem in stages. We shall work "functor-by-functor" using the definitions. If we read $\overrightarrow{\mathfrak{G}}$ from right to left and $\overrightarrow{\mathfrak{H}}$ from left to right then we see that the functors in the definitions pair-up as adjoint pairs. For four of the six functors these adjunctions are the correct way around. Two, however, are the wrong way round. Using $\overrightarrow{\mathfrak{G}}$ as our primary reference these are the $\mathfrak{C}$ and the $\mathfrak{S}$. Therefore we shall need to prove that these functors are "ineffective".

Lemma 3.33. There is a natural isomorphism of covariant functors $\overrightarrow{\mathcal{D}} \rightarrow \overrightarrow{\mathcal{E}}$

$$
\mathfrak{B C R} \overrightarrow{\mathfrak{G}} \mathfrak{S} \mathfrak{B X} \cong \mathfrak{R} \overrightarrow{\mathfrak{G}} \mathfrak{S} \mathfrak{B X} .
$$

Proof. Let $K$ be an object in $\overrightarrow{\mathcal{D}}$ and consider $\dot{\mathfrak{G}} \mathfrak{S} \mathfrak{B X}(K)$. The functors $\mathfrak{S}$ and $\mathfrak{B}$ are simply the inclusions of full subcategories and so the resulting object in $\overrightarrow{\mathcal{E}}$ has initial class

$$
\mathfrak{G}(K) \rightarrow \mathfrak{G D}\left(K_{k}\right)
$$

Using our earlier notation, the reduction of this has initial class

$$
\mathfrak{G}(K) \rightarrow \overline{\mathfrak{G}} \overline{\mathfrak{D}}\left(\bar{K}_{k}\right) .
$$

We claim that this object in $\overrightarrow{\mathcal{E}}$ is actually an object in $\overrightarrow{\mathcal{E}}$.

As $\mathfrak{X}(K)$ is an object in $\overrightarrow{\mathcal{D}}$, the natural morphism

$$
K \rightarrow \underset{k}{\lim } \mathfrak{D}\left(K_{k}\right)
$$

is an isomorphism. Since $\mathfrak{G}$ has a left adjoint, it preserves limits and so the limit in $\mathcal{E}$ exists and the natural morphism

$$
\mathfrak{G}(K) \rightarrow \underset{k}{\lim } \mathfrak{G} \mathfrak{D}\left(K_{k}\right)
$$

is an isomorphism. To complete the proof we just need to show that this limit is unchanged upon passing to the reduction.

Let $\left\{E_{\mu}\right\}$ be a directed family of objects in $\mathcal{E}$ with limit $E$ and canonical morphisms $e_{\mu}: E \rightarrow E_{\mu}$. For each $\mu$ in the indexing set, let $e_{\mu}=m_{\mu} e_{\mu}^{\prime}$ be the (extremal epi, mono)-factorisation of $e_{\mu}$ with intervening object $\tilde{E}_{\mu}$. As $\mathcal{E}$ is an (extremal epi, mono) category the family $\left\{\tilde{E}_{\mu}\right\}$ is directed under the same ordering as on the original family. Let $\tilde{E}$ be the limit of this family with $\tilde{e}_{\mu}: \tilde{E} \rightarrow \tilde{E}_{\mu}$ the canonical morphisms. 
The morphisms $e_{\mu}^{\prime}: E \rightarrow \tilde{E}_{\mu}$ define a morphism $e^{\prime}: E \rightarrow \tilde{E}$ such that $\tilde{e}_{\mu} e^{\prime}=e_{\mu}^{\prime}$. The morphisms $m_{\mu} \tilde{e}_{\mu}: \tilde{E} \rightarrow E_{\mu}$ define a morphism $m: \tilde{E} \rightarrow E$ such that $e_{\mu} m=$ $m_{\mu} \tilde{e}_{\mu}$.

Consider $m e^{\prime}: E \rightarrow E$. As $E$ is the limit of the family $\left\{E_{\mu}\right\}$ this morphism is completely determined by the compositions $e_{\mu} m e^{\prime}$. This simplifies as follows.

$$
e_{\mu} m e^{\prime}=m_{\mu} \tilde{e}_{\mu} e^{\prime}=m_{\mu} e_{\mu}^{\prime}=e_{\mu} .
$$

Hence $m e^{\prime}$ is the identity on $E$. Conversely, consider $e^{\prime} m: \tilde{E} \rightarrow \tilde{E}$. Similarly, this is completely determined by $\tilde{e}_{\mu} e^{\prime} m$ which in turn is determined by $m_{\mu} \tilde{e}_{\mu} e^{\prime} m$ as $m_{\mu}$ is a monomorphism. This simplifies as follows.

$$
m_{\mu} \tilde{e}_{\mu} e^{\prime} m=m_{\mu} e_{\mu}^{\prime} m=e_{\mu} m=m_{\mu} \tilde{e}_{\mu} .
$$

Hence $e^{\prime} m$ is the identity on $\tilde{E}$. Thus $E$ and $\tilde{E}$ are naturally isomorphic.

Applying this to the case in hand, we see that the natural morphism

$$
\mathfrak{G}(K) \rightarrow \underset{k}{\lim } \overline{\mathfrak{G}} \overline{\mathfrak{D}} \overline{\left(K_{k}\right)}
$$

is an isomorphism and hence $\mathfrak{R} \overrightarrow{\mathfrak{G}} \mathfrak{S} \mathfrak{B X}(K)$ is actually an object in $\overrightarrow{\mathcal{E}}$, albeit viewed as an object in $\overrightarrow{\mathcal{E}}$. Hence applying $\mathfrak{B C}$ to this object results in the same object again. A similar result holds for morphisms and thus there is a natural isomorphism

$$
\mathfrak{B C R} \cdot \overrightarrow{\mathfrak{G}} \mathfrak{S} \mathfrak{B X} \cong \mathfrak{R} \overrightarrow{\mathfrak{G}} \mathfrak{S} \mathfrak{B X}
$$

as required.

Corollary 3.34. There is a natural isomorphism of bifunctors $\overrightarrow{\mathcal{E}} \times \overrightarrow{\mathcal{D}} \rightarrow$ Set

$$
\overrightarrow{\mathcal{E}}(-, \overrightarrow{\mathfrak{G}}(-)) \cong \dot{\overrightarrow{\mathcal{D}}}(\overrightarrow{\mathfrak{H}} \mathfrak{S} \mathfrak{B}(-), \mathfrak{S} \mathfrak{B X}(-)) .
$$

Proof. Lemma 3.33 provides us with the crucial step in the following chain of natural isomorphisms.

$$
\begin{array}{rlrl}
\overrightarrow{\mathcal{E}}(-, \overrightarrow{\mathfrak{G}}(-)) & =\overrightarrow{\mathcal{E}}(-, \mathfrak{C} \mathfrak{R} \overrightarrow{\mathfrak{G}} \mathfrak{S} \mathfrak{B X}(-)) & & \text { definition of } \overrightarrow{\mathfrak{G}}, \\
& =\overrightarrow{\mathcal{E}}(\mathfrak{B}(-), \mathfrak{B C} \mathfrak{\mathfrak { R } \mathfrak { G }} \mathfrak{S} \mathfrak{B X}(-)) & \overrightarrow{\mathcal{E}} \text { full subcategory of } \overrightarrow{\mathcal{E}}, \\
& \cong \overrightarrow{\mathcal{E}}(\mathfrak{B}(-), \mathfrak{R} \overrightarrow{\mathfrak{G}} \mathfrak{S B X}(-)) & & \text { by Lemma } 3.33, \\
& \cong \overrightarrow{\overrightarrow{\mathcal{D}}}(\overrightarrow{\mathfrak{H}} \mathfrak{S} \mathfrak{B}(-), \mathfrak{S} \mathfrak{B X}(-)) & & \text { by the adjunction pairings } \\
& & \mathfrak{S} \dashv \mathfrak{R} \text { and } \overrightarrow{\mathfrak{H}} \dashv \overrightarrow{\mathfrak{G}}
\end{array}
$$

(the latter by Proposition 3.6).

We now reach the second point where the adjunction is the wrong way around: we have $\mathfrak{S} \neg \mathfrak{R}$ but $\mathfrak{S}$ currently appears on the right. When dealing with the problematic $\mathfrak{C}$ our strategy was to show that it was essentially not there. Our strategy here is instead to show that there is an "invisible" $\mathfrak{R}$. 
Lemma 3.35. There is a natural isomorphism of covariant functors $\overrightarrow{\mathcal{E}} \rightarrow \overrightarrow{\overrightarrow{\mathcal{D}}}$

$$
\overrightarrow{\mathfrak{H}} \mathfrak{S} \mathfrak{B} \cong \mathfrak{S} \mathfrak{R} \overrightarrow{\mathfrak{H}} \mathfrak{S} \mathfrak{B} .
$$

Proof. Let $K$ be an object in $\overrightarrow{\mathcal{E}}$. As $\mathfrak{B}$ and $\mathfrak{S}$ are simply the inclusions of full subcategories, $\overrightarrow{\mathfrak{H}} \mathfrak{S} \mathfrak{B}(K)$ is the object in $\overrightarrow{\overrightarrow{\mathcal{D}}}$ with initial class

$$
\mathfrak{H}(|K|) \rightarrow \mathfrak{H}\left(K_{k}\right) .
$$

Since $K$ is an object in $\overrightarrow{\mathcal{E}}$, it has an initial class of $\mathcal{E}$-morphisms $|K| \rightarrow K_{k}$ which are extremal epimorphisms. Hence by assumption on $\mathfrak{H}$, the corresponding morphisms $\mathfrak{H}(|K|) \rightarrow \mathfrak{H}\left(K_{k}\right)$ are also extremal epimorphisms. Thus the filtered object $\overrightarrow{\mathfrak{H}} \mathfrak{S} \mathfrak{B}(K)$ is already reduced. That is to say, there is a natural isomorphism

$$
\mathfrak{S} \mathfrak{R} \ddot{\mathfrak{H}} \mathfrak{S} \mathfrak{B} \cong \ddot{\mathfrak{H}} \mathfrak{S} \mathfrak{B} \text {. }
$$

Corollary 3.36. There is a natural isomorphism of bifunctors $\overrightarrow{\mathcal{E}} \times \overrightarrow{\mathcal{D}} \rightarrow$ Set

$$
\overrightarrow{\mathcal{E}}(-, \overrightarrow{\mathfrak{G}}(-)) \cong \overrightarrow{\mathcal{D}}(\overrightarrow{\mathfrak{H}}(-),-) .
$$

That is to say, $\overrightarrow{\mathfrak{H}} \multimap \overrightarrow{\mathfrak{G}}$.

Proof. Lemma 3.35 provides us with the crucial step in the following chain of natural isomorphisms.

$$
\begin{aligned}
\overrightarrow{\mathcal{E}}(-, \overrightarrow{\mathfrak{G}}(-)) & \cong \overrightarrow{\overrightarrow{\mathcal{D}}}(\overrightarrow{\mathfrak{H}} \mathfrak{S} \mathfrak{B}(-), \mathfrak{S} \mathfrak{B X}(-)) & & \text { by Corollary } 3.34 \\
& \cong \overrightarrow{\overrightarrow{\mathcal{D}}}(\mathfrak{S} \mathfrak{R} \overrightarrow{\mathfrak{H}} \mathfrak{S} \mathfrak{B}(-), \mathfrak{S} \mathfrak{B X}(-)) & & \text { by Lemma } 3.35 \\
& \cong \overrightarrow{\mathcal{D}}(\mathfrak{R} \overrightarrow{\mathfrak{H}} \mathfrak{S} \mathfrak{B}(-), \mathfrak{B X}(-)) & & \overrightarrow{\mathcal{D}} \text { full subcategory of } \overrightarrow{\mathcal{D}} \\
& \cong \overrightarrow{\mathcal{D}}(\mathfrak{Y} \mathfrak{C} \mathfrak{R} \overrightarrow{\mathfrak{H}} \mathfrak{S} \mathfrak{B}(-),-) & & \text { by the adjunction pairings } \\
& =\overrightarrow{\mathcal{D}}(\overrightarrow{\mathfrak{H}}(-),-) & & \mathfrak{C}-\mathfrak{B} \text { and } \mathfrak{Y} \dashv \mathfrak{X}
\end{aligned}
$$

This completes the proof of Theorem 3.32.

\subsection{Inductive Filtrations}

We have focused in this section on projective filtrations; that is, filtrations defined by morphisms out of a particular object. Using the standard categorical idea of duality we can 'op' everything in this section to obtain results on filtrations defined by morphisms into a particular object. We call these filtrations inductive filtrations. Our main interest in inductive filtrations arises from the obvious fact that a contravariant functor takes an inductive filtration to a projective one; therefore if we have a contravariant functor and wish to end up with projectively filtered objects then the correct starting point is inductively filtered ones. All of our results dualise with no difficulty and we shall use them in the following without further comment. We shall denote the inductive versions of the various categories of projectively filtered objects 
by the corresponding left-pointing arrow. Thus $\stackrel{\leftrightarrow}{\mathcal{D}}$ is the category of inductively filtered objects of $\mathcal{D}, \stackrel{\leftarrow-}{\mathcal{D}}$ is the category of reduced inductively filtered objects of $\mathcal{D}$, and $\overleftarrow{\mathcal{D}}$ is the category of inductively iso-filtered objects of $\mathcal{D}$

\section{Filtered Tall-Wraith Monoids}

We now bring together the work of the previous two sections; that is, we explain the interaction between Tall-Wraith monoids and filtrations. Let $\mathcal{V}^{*}$ be a variety of graded algebras (with grading set $Z$ ) and $\mathcal{D}$ a complete, co-complete, extremally co-well-powered, (extremal epi, mono) category. Let $\overrightarrow{\mathcal{D}} \mathcal{V}^{* c}$ be the category of co- $\mathcal{V}^{*}$ algebra objects in $\overrightarrow{\mathcal{D}}$. Co- $\mathcal{V}^{*}$-algebra objects in $\overrightarrow{\mathcal{D}}$ represent covariant functors $\overrightarrow{\mathcal{D}} \rightarrow$ $\mathcal{V}^{*}$. Recall from Theorem 2.31 that $\mathcal{V}^{*}$ is also a complete, co-complete, extremally co-well-powered, (extremal epi, mono) category and so we can lift these to functors $\overrightarrow{\mathcal{D}} \rightarrow \overrightarrow{\mathcal{V}^{*}}$ using the first construction in Definition 3.30. In particular we can take $\mathcal{D}=\mathcal{V}^{*}$ whence we obtain functors $\overrightarrow{\mathcal{V}^{*}} \rightarrow \overrightarrow{\mathcal{V}^{*}}$.

The category of all covariant functors $\overrightarrow{\mathcal{V}^{*}} \rightarrow \overrightarrow{\mathcal{V}^{*}}$ has an obvious monoidal structure coming from composition which we would like to transfer to $\overrightarrow{\mathcal{V}^{*}} \mathcal{V}^{* c}$. In the more general situation, we can consider the composition of a functor $\overrightarrow{\mathcal{D}} \rightarrow \overrightarrow{\mathcal{V}^{*}}$ with a functor $\overrightarrow{\mathcal{V}^{*}} \rightarrow \overrightarrow{\mathcal{V}^{*}}$ and we would like to show that this transfers to a pairing $\overrightarrow{\mathcal{D}} \mathcal{V}^{* c} \times \overrightarrow{\mathcal{V}^{*}} \mathcal{V}^{* c} \rightarrow$ $\overrightarrow{\mathcal{D}} \mathcal{V}^{* c}$. The groundwork of the previous section allows us to do this.

Definition 4.1. We say that a covariant functor $\overrightarrow{\mathcal{D}} \rightarrow \overrightarrow{\mathcal{V}^{*}}$ is representable if it is isomorphic to a functor of the form $\overrightarrow{G_{*}}$ for a co- $\mathcal{V}^{*}$-algebra object, $G$, in $\overrightarrow{\mathcal{D}}$. We write $\operatorname{Cov} \operatorname{Rep}\left(\overrightarrow{\mathcal{D}}, \overrightarrow{\mathcal{V}^{*}}\right)$ for the category of such functors.

It follows from the definition that the lift functor given by the first construction in Definition 3.30, $\operatorname{Cov} \operatorname{Rep}\left(\overrightarrow{\mathcal{D}}, \mathcal{V}^{*}\right) \rightarrow \operatorname{Cov} \operatorname{Rep}\left(\overrightarrow{\mathcal{D}}, \overrightarrow{\mathcal{V}^{*}}\right)$, is dense.

Proposition 4.2. The functor $\left(\overrightarrow{\mathcal{D}} \mathcal{V}^{* c}\right)^{\text {op }} \rightarrow \operatorname{CovRep}\left(\overrightarrow{\mathcal{D}}, \overrightarrow{\mathcal{V}^{*}}\right)$, given on objects by $G \mapsto$ $\overrightarrow{G_{*}}$, is an equivalence of categories.

Proof. The standard Yoneda argument gives us an equivalence between the categories $\left(\overrightarrow{\mathcal{D}} \mathcal{V}^{* c}\right)^{\text {op }}$ and $\operatorname{Cov} \operatorname{Rep}\left(\overrightarrow{\mathcal{D}}, \mathcal{V}^{*}\right)$ so we focus on the lift functor

$$
\operatorname{CovRep}\left(\overrightarrow{\mathcal{D}}, \mathcal{V}^{*}\right) \rightarrow \operatorname{Cov} \operatorname{Rep}\left(\overrightarrow{\mathcal{D}}, \overrightarrow{\mathcal{V}^{*}}\right)
$$

We define a functor $\operatorname{CovRep}\left(\overrightarrow{\mathcal{D}}, \overrightarrow{\mathcal{V}^{*}}\right) \rightarrow \operatorname{CovRep}\left(\overrightarrow{\mathcal{D}}, \mathcal{V}^{*}\right)$ by post composition with the forgetful functor $\mathfrak{Y}: \overrightarrow{\mathcal{V}^{*}} \rightarrow \mathcal{V}^{*}$. Recall that $\mathfrak{Y}=\mathfrak{U} \mathfrak{S} \mathfrak{B}$.

The category $\overrightarrow{\mathcal{D}}$ is co-complete by Corollary 3.24 and so by Corollary 2.30 a functor, say $\mathfrak{G}$, in $\operatorname{Cov} \operatorname{Rep}\left(\overrightarrow{\mathcal{D}}, \mathcal{V}^{*}\right)$ has a left adjoint. Thus Lemma 3.33 applies and after composing with $\mathfrak{U} \mathfrak{S}$ we have a natural isomorphism

$$
\mathfrak{Y} \overrightarrow{\mathfrak{G}}=\mathfrak{U} \mathfrak{S} \mathfrak{B} \overrightarrow{\mathfrak{G}} \cong \mathfrak{U} \mathfrak{S} \mathfrak{R} \overrightarrow{\mathfrak{G}} \mathfrak{S} \mathfrak{B} \mathfrak{X}
$$

Since $\mathfrak{R}$ does not change the underlying object in $\mathcal{V}^{*}, \mathfrak{U} \mathfrak{S} \mathfrak{R}=\mathfrak{U}$. Hence there is a 
natural isomorphism

$$
\mathfrak{Y} \overrightarrow{\mathfrak{G}} \cong \mathfrak{U} \overrightarrow{\mathfrak{G}} \mathfrak{S} \mathfrak{B X} .
$$

From the definition of $\overrightarrow{\mathfrak{G}}$ we see that $\vec{U} \overrightarrow{\mathfrak{G}}=\mathfrak{G} \mathfrak{U}$ and from the definition of $\mathfrak{X}$ we see that $\mathfrak{U S \mathfrak { X }}$ is the identity on $\overrightarrow{\mathcal{V}^{*}}$. Thus we have a natural isomorphism

$$
\mathfrak{Y} \overrightarrow{\mathfrak{G}} \cong \mathfrak{G} .
$$

A similar story occurs on morphisms (i.e. natural transformations) and so it is clear that the composition

$$
\operatorname{CovRep}\left(\overrightarrow{\mathcal{V}^{*}}, \mathcal{V}^{*}\right) \rightarrow \operatorname{CovRep}\left(\overrightarrow{\mathcal{V}^{*}}, \overrightarrow{\mathcal{V}^{*}}\right) \rightarrow \operatorname{CovRep}\left(\overrightarrow{\mathcal{V}^{*}}, \mathcal{V}^{*}\right)
$$

is naturally isomorphic to the identity on $\operatorname{CovRep}\left(\overrightarrow{\mathcal{V}^{*}}, \mathcal{V}^{*}\right)$. The functor

$$
\operatorname{CovRep}\left(\overrightarrow{\mathcal{V}^{*}}, \overrightarrow{\mathcal{V}^{*}}\right) \rightarrow \operatorname{CovRep}\left(\overrightarrow{\mathcal{V}^{*}}, \mathcal{V}^{*}\right)
$$

is therefore dense. Using the fact that $\operatorname{Cov} \operatorname{Rep}\left(\overrightarrow{\mathcal{V}^{*}}, \mathcal{V}^{*}\right) \rightarrow \operatorname{Cov} \operatorname{Rep}\left(\overrightarrow{\mathcal{V}^{*}}, \overrightarrow{\mathcal{V}^{*}}\right)$ is dense, we also see that the above functor is full. Furthermore, it is faithful because the forgetful functor, $\mathfrak{U}: \overrightarrow{\mathcal{V}^{*}} \rightarrow \mathcal{V}^{*}$, is faithful. Hence it is an equivalence of categories.

Exactly as previously, we wish to use this equivalence to define a monoidal structure on $\overrightarrow{\mathcal{V}^{*}} \mathcal{V}^{* c}$ which corresponds to composition of functors.

Theorem 4.3. Under the stated assumptions on $\mathcal{D}$, the composition of representable functors, in the sense of Definition 4.1, is representable.

The proof of this depends on the results of the previous section. We therefore need to show that they apply. Let $G$ be a co- $\mathcal{V}^{*}$-algebra object in $\overrightarrow{\mathcal{D}}$. This represents the covariant functor $G_{*}$ and this functor has a left adjoint $G$ ! by Corollary 2.30 .

Lemma 4.4. The functor $G_{!}: \mathcal{V}^{*} \rightarrow \overrightarrow{\mathcal{D}}$ preserves extremal epimorphisms.

Proof. Let $f: V_{1} \rightarrow V_{2}$ be an extremal epimorphism in $\mathcal{V}^{*}$. As $G$ ! is a left adjoint it takes epimorphisms to epimorphisms and thus $G_{!}(f)$ is an epimorphism. The crucial part is therefore to show that it is extremal.

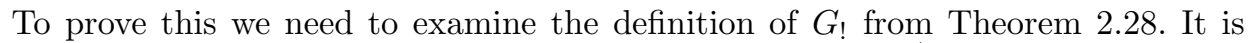
defined using the left adjoint of the underlying functor $\left|G_{*}\right|: \overrightarrow{\mathcal{D}} \rightarrow \operatorname{Set}^{Z}$; let us write this adjoint as $|G|_{!}$. The relationship is that there is a co-equaliser sequence in $\overrightarrow{\mathcal{D}}$, natural in $V$ in $\mathcal{V}^{*}$,

$$
|G|_{!}\left(\left|\mathfrak{F}_{\mathcal{V}^{*}}(|V|)\right|\right) \underset{s_{V}}{\stackrel{r_{V}}{\longrightarrow}}|G|_{!}(|V|) \stackrel{p_{V}}{\longrightarrow} G_{!}(V)
$$

Therefore for a $\mathcal{V}^{*}$-morphism $f: V_{1} \rightarrow V_{2}$ we have a diagram in $\overrightarrow{\mathcal{D}}$ with the obvious 
commuting properties:

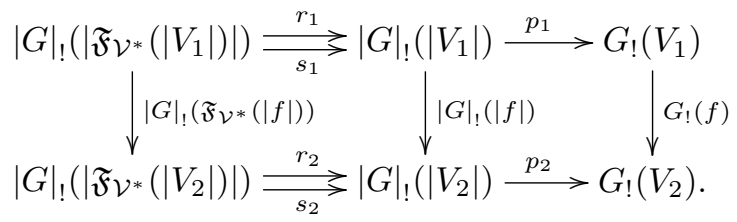

We know that $G_{!}(f)$ is an epimorphism. Suppose that we have a factorisation $G_{!}(f)=$ me with $m: K \rightarrow G_{!}\left(V_{2}\right)$ a monomorphism. As $f$ is an extremal epimorphism, Theorem 2.31 says that $|f|:\left|V_{1}\right| \rightarrow\left|V_{2}\right|$ is an epimorphism on the underlying objects in $\operatorname{Set}^{Z}$ and thus admits a section, say $g:\left|V_{2}\right| \rightarrow\left|V_{1}\right|$. Hence there is a $\overrightarrow{\mathcal{D}}$ morphism $h:|G|_{!}\left(\left|V_{2}\right|\right) \rightarrow K$ given by

$$
|G|_{!}\left(\left|V_{2}\right|\right) \stackrel{|G|_{!}(g)}{\longrightarrow}|G|_{!}\left(\left|V_{1}\right|\right) \stackrel{p_{1}}{\longrightarrow} G_{!}\left(V_{1}\right) \stackrel{e}{\longrightarrow} K .
$$

Then

$$
m h=\operatorname{mep}_{1}|G|_{!}(g)=G_{!}(f) p_{1}|G|_{!}(g)=p_{2}|G|_{!}(|f|)|G|_{!}(g)=p_{2}
$$

and so we have a factorisation of $p_{2}$ as $m h$. Thus $m h r_{2}=m h s_{2}$ and so, as $m$ is a monomorphism, $h r_{2}=h s_{2}$. Thus $h$ co-equalises $r_{2}$ and $s_{2}$ so factors uniquely through the co-equaliser which is $G_{!}\left(V_{2}\right)$. That is, there is a unique $\overrightarrow{\mathcal{D}}$-morphism $k: G_{!}\left(V_{2}\right) \rightarrow$ $K$ such that $k p_{2}=h$. Then $m k p_{2}=m h=p_{2}$ whence, as $p_{2}$ is an epimorphism, $m k$ is the identity on $G_{!}\left(V_{2}\right)$. Thus $m$ is a monomorphism which admits a section and hence is an isomorphism.

In conclusion, $G_{!}(f)$ is an extremal epimorphism.

Thus Theorem 3.32 applies to the pair $\left(G_{!}, G_{*}\right)$.

Corollary 4.5. The functor $\overrightarrow{G_{!}}$is left adjoint to $\overrightarrow{G_{*}}$.

We extend the functor $\overrightarrow{G_{!}}: \overrightarrow{\mathcal{V}^{*}} \rightarrow \overrightarrow{\mathcal{D}}$ to $\vec{G}_{!}^{Z}: \overrightarrow{\mathcal{V}}^{Z} \rightarrow \overrightarrow{\mathcal{D}}^{Z}$ in the obvious way. As $\overrightarrow{G_{!}}$is a left adjoint it preserves co-products and thus so does $\vec{G}_{!}^{Z}$. This therefore lifts to a functor $\vec{G}_{!}^{c}: \overrightarrow{\mathcal{V}}^{*} \mathcal{V}^{* c} \rightarrow \overrightarrow{\mathcal{D}} \mathcal{V}^{* c}$.

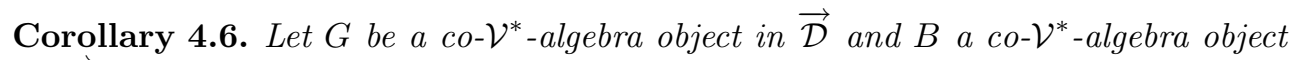
in $\overrightarrow{\mathcal{V}^{*}}$. The composition

$$
B_{*} \overrightarrow{G_{*}}: \overrightarrow{\mathcal{D}} \rightarrow \mathcal{V}^{*}
$$

is representable with representing object $\vec{G}_{!}^{c}(B)$.

As we are considering a functor into $\mathcal{V}^{*}$ we are using the usual meaning of "representable" here.

Proof. Both $B_{*}$ and $\overrightarrow{G_{*}}$ have left adjoints and so their composition has a left adjoint. It is therefore representable by Corollary 2.30. That the representing object is as given is straightforward to see.

As $B_{*}$ is a right adjoint, it preserves monomorphisms. Hence Theorem 3.31 applies to the pair $\left(B_{*}, G_{*}\right)$ to prove Theorem 4.3 . 


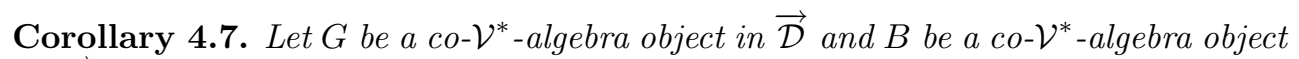
in $\overrightarrow{\mathcal{V}^{*}}$. The composition

$$
\overrightarrow{B_{*}} \overrightarrow{G_{*}}: \overrightarrow{\mathcal{D}} \rightarrow \overrightarrow{\mathcal{V}^{*}}
$$

is representable with representing object $\vec{G}_{!}^{c}(B)$.

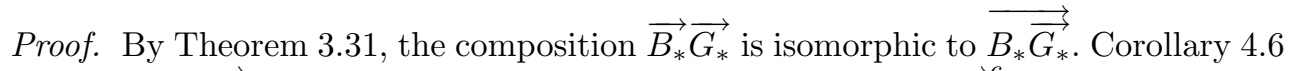
says that $B_{*} \overrightarrow{G_{*}}$ is representable (in the traditional sense) by $\overrightarrow{G !}_{!}^{c}(B)$ and hence, by definition, $\overrightarrow{B_{*}} \overrightarrow{G_{*}}$ is representable with representing object $\vec{G}_{!}^{c}(B)$.

By putting $\mathcal{D}=\mathcal{V}^{*}$ we have a pairing $\overrightarrow{\mathcal{V}^{*}} \mathcal{V}^{* c} \times \overrightarrow{\mathcal{V}^{*}} \mathcal{V}^{* c} \rightarrow \overrightarrow{\mathcal{V}^{*}} \mathcal{V}^{* c}$ corresponding to composition of functors. The next step in showing that $\overrightarrow{\mathcal{V}^{*}} \mathcal{V}^{* c}$ is monoidal is to prove that the identity functor on $\overrightarrow{\mathcal{V}^{*}}$ is representable.

Lemma 4.8. The discrete filtration functor, $\mathfrak{D}: \mathcal{V}^{*} \rightarrow \overrightarrow{\mathcal{V}^{*}}$, lifts to a functor

$$
\mathfrak{D}^{c}: \mathcal{V}^{*} \mathcal{V}^{* c} \rightarrow \overrightarrow{\mathcal{V}^{*}} \mathcal{V}^{* c}
$$

For a co- $\mathcal{V}^{*}$-algebra object, $B$, in $\mathcal{V}^{*}$ and an object, $Y$, in $\overrightarrow{\mathcal{V}^{*}}$ there is an isomorphism of $\mathcal{V}^{*}$-algebras, natural in both $B$ and $Y$,

$$
\overrightarrow{\mathcal{V}^{*}}\left(\mathfrak{D}^{c}(B), Y\right) \cong \mathcal{V}^{*}(B, \mathfrak{Y}(Y))
$$

Proof. There is an obvious extension

$$
\mathfrak{D}^{Z}: \mathcal{V}^{* Z} \rightarrow \overrightarrow{\mathcal{V}}^{Z}
$$

which is left adjoint to the corresponding extension of the forgetful functor

$$
\mathfrak{Y}^{Z}: \overrightarrow{\mathcal{V}}^{Z} \rightarrow \mathcal{V}^{* Z}
$$

As $\mathfrak{D}^{Z}$ is a left adjoint, it preserves co-products and hence takes co-algebra objects to co-algebra objects. It therefore extends to $\mathfrak{D}^{c}: \mathcal{V}^{*} \mathcal{V}^{* c} \rightarrow \overrightarrow{\mathcal{V}^{*}} \mathcal{V}^{* c}$.

Let $B$ be a co- $\mathcal{V}^{*}$-algebra object in $\mathcal{V}^{*}$ and $Y$ an object in $\overrightarrow{\mathcal{V}^{*}}$. The underlying object in $\operatorname{Set}^{Z}$ of $\overrightarrow{\mathcal{V}^{*}}\left(\mathfrak{D}^{c}(B), Y\right)$ is

$$
\begin{aligned}
z \mapsto & \overrightarrow{\mathcal{V}^{*}}\left(\left|\mathfrak{D}^{c}(B)\right|(z), Y\right) \\
& =\overrightarrow{\mathcal{V}^{*}}\left(\mathfrak{D}^{Z}(|B|)(z), Y\right) \\
& =\overrightarrow{\mathcal{V}^{*}}(\mathfrak{D}(|B|(z)), Y) \\
& \cong \mathcal{V}^{*}(|B|(z), \mathfrak{Y}(Y)) .
\end{aligned}
$$

Thus there is a bijection from the underlying object in $\operatorname{Set}^{Z}$ of $\overrightarrow{\mathcal{V}^{*}}\left(\mathfrak{D}^{c}(B), Y\right)$ to that of $\mathcal{V}^{*}(B, \mathfrak{Y}(Y))$. It is clear that this is an isomorphism of $\mathcal{V}^{*}$-algebras.

Proposition 4.9. Let I be the unit of the Tall-Wraith monoidal structure on $\mathcal{V}^{*} \mathcal{V}^{* c}$. The co- $\mathcal{V}^{*}$-algebra object in $\overrightarrow{\mathcal{V}^{*}}, \mathfrak{D}^{c}(I)$, represents the identity functor on $\overrightarrow{\mathcal{V}^{*}}$.

Recall that as the functor $\mathcal{V}^{*} \mathcal{V}^{* c} \rightarrow \operatorname{CovRep}\left(\mathcal{V}^{*}, \mathcal{V}^{*}\right)$ is strong monoidal, $I$ represents the identity functor on $\mathcal{V}^{*}$. 
Proof. Let $Y$ be an object in $\overrightarrow{\mathcal{V}^{*}}$. Using Lemma 4.8, we have the following isomorphisms of $\mathcal{V}^{*}$-algebras

$$
\overrightarrow{\mathcal{V}^{*}}\left(\mathfrak{D}^{c}(I), Y\right) \cong \mathcal{V}^{*}(I, \mathfrak{Y}(Y)) \cong \mathfrak{Y}(Y) .
$$

Thus $\mathfrak{D}^{c}(I)$ represents the forgetful functor, $\mathfrak{Y}: \overrightarrow{\mathcal{V}^{*}} \rightarrow \mathcal{V}^{*}$. We need to show that $\overrightarrow{\mathfrak{Y}}: \overrightarrow{\mathcal{V}^{*}} \rightarrow \overrightarrow{\mathcal{V}^{*}}$ is isomorphic to the identity.

Let us write out the stages in defining $\overrightarrow{\mathfrak{Y}}(Y)$. It is

$$
\begin{aligned}
& Y=\left(|Y| \rightarrow Y_{\lambda}\right) \stackrel{\mathfrak{X}}{\longmapsto}\left(Y \rightarrow \mathfrak{D}\left(Y_{\lambda}\right)\right) \\
& \stackrel{\mathfrak{B}}{\longrightarrow}\left(Y \rightarrow \mathfrak{D}\left(Y_{\lambda}\right)\right) \\
& \stackrel{\mathfrak{S}}{\longrightarrow}\left(Y \rightarrow \mathfrak{D}\left(Y_{\lambda}\right)\right) \\
& \stackrel{\mathfrak{i}}{\longmapsto}\left(|Y| \rightarrow Y_{\lambda}\right) \\
& \stackrel{\Re}{\longmapsto}\left(|Y| \rightarrow Y_{\lambda}\right) \\
& \stackrel{\mathfrak{C}}{\rightarrow}\left(|Y| \rightarrow Y_{\lambda}\right)=Y \text {. }
\end{aligned}
$$

Hence $\overrightarrow{\mathfrak{Y}}$ is the identity functor on $\overrightarrow{\mathcal{V}^{*}}$ and thus the identity functor is representable.

The final step in showing that we have a monoidal structure on $\overrightarrow{\mathcal{V}^{*}}$ is to show that the pairing and the unit satisfy the various coherences. These are automatic as these coherences are satisfied in the category of functors from $\overrightarrow{\mathcal{V}^{*}}$ to itself.

Let us gather together all the main results into one theorem. By working with the appropriate opposite categories we gain one additional result - note that this involves a lot of switching, even of the direction of the filtration; recall that we use left-pointing arrows for the categories of inductively filtered objects. By another obvious alteration, we can also have two graded varieties of algebras involved, even with different grading sets.

Theorem 4.10. Let $\mathcal{V}^{*}$ be a variety of graded algebras.

1. The category of co- $\mathcal{V}^{*}$-algebra objects in $\overrightarrow{\mathcal{V}^{*}}, \overrightarrow{\mathcal{V}^{*}} \mathcal{V}^{* c}$, has a monoidal structure with pairing

$$
\left(B_{1}, B_{2}\right) \mapsto{\overrightarrow{B_{2 !}}}^{c}\left(B_{1}\right)
$$

and unit $\mathfrak{D}^{c}(I)$.

The functor $\overrightarrow{\mathcal{V}^{*}} \mathcal{V}^{* c} \rightarrow \operatorname{CovFun}\left(\overrightarrow{\mathcal{V}^{*}}, \overrightarrow{\mathcal{V}^{*}}\right), B \mapsto \overrightarrow{B_{*}}$, is strong monoidal.

2. Let $\mathcal{D}$ be a complete, co-complete extremally co-well-powered, (extremal epi, mono) category. There is a pairing

$$
\overrightarrow{\mathcal{V}^{*}} \mathcal{V}^{* c} \times \overrightarrow{\mathcal{D}} \mathcal{V}^{* c} \rightarrow \overrightarrow{\mathcal{D}} \mathcal{V}^{* c}
$$

compatible with the monoidal structure on $\overrightarrow{\mathcal{V}^{*}} \mathcal{V}^{* c}$ and with composition of functors. 
3. Let $\mathcal{D}$ be a complete, co-complete, extremally well-powered, (epi, extremal mono) category. There is a pairing

$$
\left(\overrightarrow{\mathcal{V}^{*}} \mathcal{V}^{* c}\right)^{\text {op }} \times \overleftarrow{\mathcal{D}} \mathcal{V}^{*} \rightarrow \overleftarrow{\mathcal{D}} \mathcal{V}^{*}
$$

compatible with the monoidal structure on $\overrightarrow{\mathcal{V}^{*}} \mathcal{V}^{* c}$ and with composition of functors.

4. Let $\mathcal{W}^{*}$ be a graded variety of algebras, possibly with a different grading set to that of $\mathcal{V}^{*}$. There is a pairing

$$
\overrightarrow{\mathcal{V}^{*}} \mathcal{W}^{* c} \times \overrightarrow{\mathcal{V}^{*}} \mathcal{V}^{* c} \rightarrow \overrightarrow{\mathcal{V}^{*}} \mathcal{W}^{* c}
$$

compatible with the monoidal structure on $\overrightarrow{\mathcal{V}^{*}} \mathcal{V}^{* c}$ and with composition of functors.

Again, we shall use the notation $-\odot-$ to denote the pairing in each case.

We can deal with the variance shift on the middle pairing in the usual manner.

Proposition 4.11. There is a pairing

$$
\overrightarrow{\mathcal{V}^{*}} \mathcal{V}^{* c} \times \overleftarrow{\mathcal{D}} \mathcal{V}^{*} \rightarrow \overleftarrow{\mathcal{D}} \mathcal{V}^{*}, \quad(B, H) \mapsto B \circledast H
$$

compatible with the monoidal structure on $\overrightarrow{\mathcal{V}^{*}} \mathcal{V}^{* c}$ and a natural isomorphism

$$
\overleftarrow{\mathcal{D}} \mathcal{V}^{*}\left(B \circledast H, H^{\prime}\right) \cong \overleftarrow{\mathcal{D}} \mathcal{V}^{*}\left(H, B \odot H^{\prime}\right)
$$

for $B$ in $\overrightarrow{\mathcal{V}^{*}} \mathcal{V}^{* c}$ and $H, H^{\prime}$ in $\overleftarrow{\mathcal{D}} \mathcal{V}^{*}$

We have the obvious definition.

Definition 4.12. Let $\mathcal{V}^{*}$ be a variety of graded algebras. A Tall-Wraith $\overrightarrow{\mathcal{V}^{*}}$-monoid is a monoid in $\overrightarrow{\mathcal{V}^{*}} \mathcal{V}^{* c}$.

The remarks following the definition of a Tall-Wraith $\mathcal{V}$-monoid regarding module and co-module objects clearly apply here as well.

\section{Tall-Wraith Monoids in Algebraic Topology}

In this section we come to the heart of the matter: operations on cohomology theories. For a suitable cohomology theory we shall show that the set of unstable operations on that theory has the structure of a Tall-Wraith monoid. This encodes all of the information available except for the suspension isomorphism. We shall also show how to interpret the associated enriched Hopf ring of the co-operations.

\subsection{Operations}

We shall start by considering operations. We shall do this in a general setting. Let us start by listing the ingredients and the properties that they have to satisfy.

We have the following.

1. A category $\mathcal{E}$ which is closed under finite products.

2. A graded variety of algebras, $\mathcal{V}^{*}$, with indexing set $Z$. 
3. A filtration functor $\mathfrak{E}: \mathcal{E} \rightarrow \stackrel{\leftrightarrow}{\mathcal{E}}$.

4. A $\mathcal{V}^{*}$-algebra object, $R$, in $\mathcal{E}$.

Before stating the conditions let us introduce some notation. Using the above data we construct a contravariant functor $\overline{R^{*}}: \mathcal{E} \rightarrow \overrightarrow{\mathcal{V}^{*}}$ as the composition

$$
\mathcal{E} \stackrel{\mathfrak{E}}{\rightarrow} \stackrel{\dot{\mathcal{E}}}{\stackrel{R^{*}}{\longrightarrow}} \dot{\mathcal{V}}^{*} \stackrel{\Re}{\longrightarrow} \overrightarrow{\mathcal{V}^{*}} \stackrel{\mathfrak{C}}{\rightarrow} \overrightarrow{\mathcal{V}^{*}}
$$

The $\mathcal{V}^{*}$-algebra object, $R$, in $\mathcal{E}$ has underlying object, $|R|$, in $\mathcal{E}^{Z}$ and so for each $z \in Z$ we have an object, $|R|(z)$, in $\mathcal{E}$. As $\mathcal{E}$ is closed under finite products, for each finite subset $Z_{0} \subseteq Z$ we can form the product $\prod_{z \in Z_{0}}|R|(z)$. As $\overline{R^{*}}$ is a contravariant functor, for $z_{0} \in Z_{0}$ it takes the projection $\mathcal{E}$-morphism $\prod_{z \in Z_{0}}|R|(z) \rightarrow|R|\left(z_{0}\right)$ to a $\overrightarrow{\mathcal{V}^{*}}$-morphism

$$
\overline{R^{*}}\left(|R|\left(z_{0}\right)\right) \rightarrow \overline{R^{*}}\left(\prod_{z \in Z_{0}}|R|(z)\right)
$$

and thus, as $\overrightarrow{\mathcal{V}^{*}}$ is co-complete, we have a $\overrightarrow{\mathcal{V}^{*}}$-morphism

$$
\coprod_{z \in Z_{0}} \overline{R^{*}}(|R|(z)) \rightarrow \overline{R^{*}}\left(\prod_{z \in Z_{0}}|R|(z)\right) .
$$

We can now state our conditions on the ingredients for our construction.

1. For each finite subset $Z_{0} \subseteq Z$, the above $\overrightarrow{\mathcal{V}^{*}}$-morphism

$$
\coprod_{z \in Z_{0}} \overline{R^{*}}(|R|(z)) \rightarrow \overline{R^{*}}\left(\prod_{z \in Z_{0}}|R|(z)\right)
$$

is an isomorphism.

2. The filtration functor $\mathfrak{E}: \mathcal{E} \rightarrow \stackrel{\leftrightarrow}{\mathcal{E}}$ has the following property. The inductive filtration $\mathfrak{E}(E)$ for an object, $E$, in $\mathcal{E}$ has a final family, say $\left\{E_{\lambda}\right\}$, such that each $\mathfrak{E}\left(E_{\lambda}\right)$ is an iso-filtration.

The first condition obviously implies the following result.

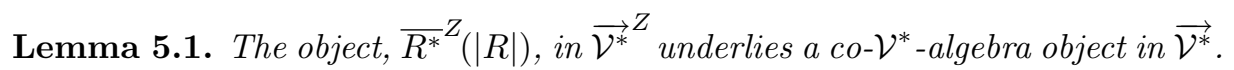

The full notation for this co- $\mathcal{V}^{*}$-algebra object in $\overrightarrow{\mathcal{V}^{*}}$ ought to be

$$
{\overline{R^{*}}}^{Z}(R)
$$

but that rapidly becomes unwieldy. As we have fixed our initial data, including $R$, we shall write ${\overline{R^{*}}}^{Z}(R)$ as just $P$. We shall also write $|P|$ for ${\overline{R^{*}}}^{Z}(|R|)$.

Proposition 5.2. Let $E$ be an object in $\mathcal{E}$. The morphism in $\operatorname{Set}^{Z}$

$$
\mathcal{E}(E,|R|) \rightarrow \overrightarrow{\mathcal{V}^{*}}\left({\overline{R^{*}}}^{Z}(|R|), \overline{R^{*}}(E)\right)=\overrightarrow{\mathcal{V}^{*}}\left(|P|, \overline{R^{*}}(E)\right)
$$

induced by $\overline{R^{*}}$ defines a morphism of objects in $\overrightarrow{\mathcal{V}^{*}}$

$$
\overline{R^{*}}(E) \rightarrow \overrightarrow{P_{*}}\left(\overline{R^{*}}(E)\right)
$$

which is natural in $E$. 
The last expression could probably do with some explanation. The co- $\mathcal{V}^{*}$-algebra object, $P$, in $\overrightarrow{\mathcal{V}^{*}}$ represents a covariant functor $P_{*}: \overrightarrow{\mathcal{V}^{*}} \rightarrow \mathcal{V}^{*}$. By the first construction of Definition 3.30 we can lift this to a covariant functor $\overrightarrow{\mathcal{V}^{*}} \rightarrow \overrightarrow{\mathcal{V}^{*}}$ which, using the notation of Definition 3.30, we write as $\overrightarrow{P_{*}}$.

Proof. For objects, $E_{1}$ and $E_{2}$, in $\mathcal{E}$, the functor $\overline{R^{*}}$ defines a morphism of sets, natural in $E_{1}$ and $E_{2}$,

$$
\mathcal{E}\left(E_{1}, E_{2}\right) \rightarrow \overrightarrow{\mathcal{V}^{*}}\left(\overline{R^{*}}\left(E_{2}\right), \overline{R^{*}}\left(E_{1}\right)\right) .
$$

For $z \in Z$ we therefore have a morphism of sets

$$
\mathcal{E}(E,|R|(z)) \rightarrow \overrightarrow{\mathcal{V}^{*}}\left(\overline{R^{*}}(|R|(z)), \overline{R^{*}}(E)\right)
$$

and thus a morphism in $\operatorname{Set}^{Z}$

$$
\mathcal{E}(E,|R|) \rightarrow \overrightarrow{\mathcal{V}^{*}}\left({\overline{R^{*}}}^{Z}(|R|), \overline{R^{*}}(E)\right)=\overrightarrow{\mathcal{V}^{*}}\left(|P|, \overline{R^{*}}(E)\right)
$$

which can be rewritten as

$$
|R|^{*}(E) \rightarrow|P|_{*}\left(\overline{R^{*}}(E)\right) .
$$

Both sides underlie $\mathcal{V}^{*}$-algebras. The structure on the left comes from the occurrence of $R$ as this is a $\mathcal{V}^{*}$-algebra object in $\mathcal{E}$. That on the right comes from the occurrence of $|P|$ as this underlies a co- $\mathcal{V}^{*}$-algebra object in $\overrightarrow{\mathcal{V}^{*}}$. This structure in turn comes from the fact that $|P|={\overline{R^{*}}}^{Z}(|R|)$ and $|R|$ underlies a $\mathcal{V}^{*}$-algebra object in $\mathcal{E}$. Both structures therefore come from the same source and so the above morphism of objects in $\mathrm{Set}^{Z}$ lifts to a morphism of $\mathcal{V}^{*}$-algebras

$$
R^{*}(E) \rightarrow P_{*}\left(\overline{R^{*}}(E)\right)
$$

which is natural in $E$.

Both sides have filtrations and we wish to compare these. The filtration on the left comes from applying the functor $\mathfrak{E}$ to $E$. That on the right comes from applying the canonical filtration functor, $\mathfrak{S} \mathfrak{B X}: \overrightarrow{\mathcal{V}^{*}} \rightarrow \overrightarrow{\mathcal{V}^{*}}$, to $\overrightarrow{R^{*}}(E)$. We shall show that the

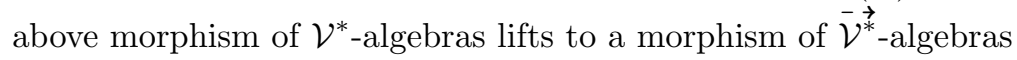

$$
\mathfrak{R} \ddot{R}^{*} \mathfrak{E}(E) \rightarrow \mathfrak{R}(\dddot{P})_{*}^{+} \mathfrak{S} \mathfrak{B X}\left(\overline{R^{*}}(E)\right) .
$$

The reduced projective filtrations on both sides are constructed from the inductive filtration $\mathfrak{E}(E)$ on $E$. Initial subclasses can be explicitly constructed as follows. We shall start with the reduced projective filtration on the left hand side of (3). Let $e: E_{e} \rightarrow E$ be in $\mathfrak{E}(E)$. Applying the contravariant functor $R^{*}$ to $e$ results in a $\mathcal{V}^{*}$ morphism $R^{*}(E) \rightarrow R^{*}\left(E_{e}\right)$. We choose an (extremal epi, mono)-factorisation of this with intervening object, $\bar{R}^{*}\left(\bar{E}_{e}\right)$, in $\mathcal{V}^{*}$. We therefore have an extremal epimorphism $R^{*}(E) \rightarrow \bar{R}^{*}\left(\bar{E}_{e}\right)$. Doing this for each $e$ in $\mathfrak{E}(E)$ defines the required initial subclass for $\mathfrak{R} \ddot{R} \vec{R}(E)$.

Now let us consider the reduced projective filtration on the right hand side. The $\overrightarrow{\mathcal{V}^{*}}$-algebra, $\overline{R^{*}}(E)$, as a projectively filtered $\mathcal{V}^{*}$-algebra, has initial subclass given by 
the $\mathcal{V}^{*}$-morphisms

$$
\left|\overline{R^{*}}(E)\right| \rightarrow \bar{R}^{*}\left(\bar{E}_{e}\right)
$$

for $e$ in $\mathfrak{E}(E)$. An initial subclass for the canonical filtration on $\overline{R^{*}}(E)$ is therefore given by the $\overrightarrow{\mathcal{V}^{*}}$-morphisms

$$
\overline{R^{*}}(E) \rightarrow \mathfrak{D}\left(\bar{R}^{*}\left(\bar{E}_{e}\right)\right)
$$

Therefore an initial subclass for the reduced projective filtration on the right hand side of $(3)$ is given by the $\mathcal{V}^{*}$-morphisms

$$
P_{*}\left(\overline{R^{*}}(E)\right) \rightarrow P_{*} \mathfrak{D}\left(\overline{R^{*}}\left(\overline{E_{e}}\right) \bar{T}\right) .
$$

Let $e$ be in $\mathfrak{E}(E)$. We claim that the following is a commutative diagram in $\mathcal{V}^{*}$.

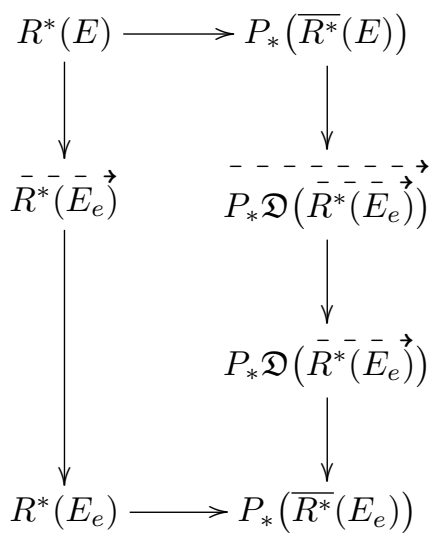

The lower horizontal morphism comes from applying (2) with $E_{e}$ in place of $E$. The bottom vertical morphism on the right arises as follows. From the definition of $\bar{R}^{*}\left(\bar{E}_{e}\right)$ there is a morphism

$$
\bar{R}^{*}\left(\bar{E}_{e}\right) \rightarrow R^{*}\left(E_{e}\right)
$$

From the definition of $\overline{R^{*}}\left(E_{e}\right)$ there is a morphism

$$
R^{*}\left(E_{e}\right) \rightarrow\left|\overline{R^{*}}\left(E_{e}\right)\right|
$$

whence we have a morphism

$$
\mathfrak{D}\left(\bar{R}^{*}\left(\bar{E}_{e}\right)\right) \rightarrow \mathfrak{D}\left(R^{*}\left(E_{e}\right)\right) \rightarrow \overline{R^{*}}\left(E_{e}\right)
$$

to which we apply $P_{*}$.

It is routine to check that the composition on the right hand side is $P_{*}\left(\overline{R^{*}}(e)\right)$ and thus the above diagram commutes by naturality of (2).

We claim that our assumption on $\mathfrak{E}$ ensures that the bottom vertical morphism on the right hand side is a monomorphism. As $R^{*}$ is representable, it is one of a mutually right adjoint pair and thus takes epi-sinks to mono-sources. Hence $R^{*}\left(E_{e}\right)$ 
is a mono-source for $\ddot{R}^{*} \mathfrak{E}\left(E_{e}\right)$. This obviously remains true after applying the reduction functor and hence the natural morphism $R^{*}\left(E_{e}\right) \rightarrow\left|\overline{R^{*}}\left(E_{e}\right)\right|$ is a monomorphism. Since $\bar{R}^{*}\left(\bar{E}_{e}\right) \rightarrow R^{*}\left(E_{e}\right)$ is a monomorphism by construction we therefore have a monomorphism $\bar{R}^{*}\left(\bar{E}_{e}\right) \rightarrow\left|\overline{R^{*}}\left(E_{e}\right)\right|$. This morphism underlies the $\overrightarrow{\mathcal{V}^{*}}$-morphism $\mathfrak{D}\left(\bar{R}^{*}\left(\bar{E}_{e}\right)\right) \rightarrow \overline{R^{*}}\left(E_{e}\right)$ which is therefore a monomorphism as the forgetful functor is faithful. Since $P_{*}$ is a right adjoint it takes monomorphisms to monomorphisms.

Thus the composition of the lower two vertical morphisms on the right hand side is a monomorphism and so as $\mathcal{V}^{*}$ is an (extremal epi, mono) category we have a horizontal morphism

$$
\overrightarrow{R^{*}}\left(\bar{E}_{e}\right) \rightarrow P_{*} \mathfrak{D}\left(\bar{R}^{*}\left(\bar{E}_{e} \vec{t}\right)\right.
$$

fitting into the diagram.

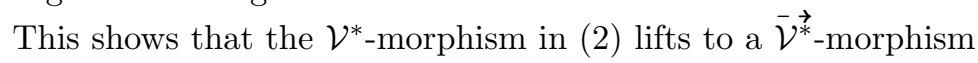

$$
\mathfrak{R} \vec{R}^{*} \mathfrak{E}(E) \rightarrow \mathfrak{R} \vec{P}_{*} \mathfrak{S} \mathfrak{B X}\left(\overline{R^{*}}(E)\right)
$$

as required. Applying $\mathfrak{C}$ to both sides results in a $\overrightarrow{\mathcal{V}^{*}}$-morphism

$$
\mathfrak{C} \mathfrak{R} \vec{R}^{*} \mathfrak{E}(E) \rightarrow \mathfrak{C} \mathfrak{R} \vec{P}_{*} \mathfrak{S} \mathfrak{B X}\left(\overline{R^{*}}(E)\right) .
$$

By definition, this is a morphism of $\overrightarrow{\mathcal{V}^{*}}$-algebras

$$
\overline{R^{*}}(E) \rightarrow \overline{P_{*}}\left(\overline{R^{*}}(E)\right)
$$

as required. It is obvious from its construction that it is natural in $E$.

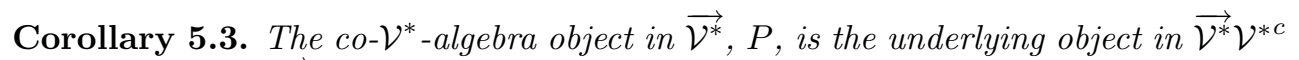
of a Tall-Wraith $\overrightarrow{\mathcal{V}^{*}}$-monoid.

For an object, $E$, in $\mathcal{E}, \overline{R^{*}}(E)$ is a module for this monoid.

Proof. Using the adjunction $\overrightarrow{G_{!}} \dashv \overrightarrow{G_{*}}$ from Corollary 4.5, the morphism from Proposition 5.2 defines a morphism of $\overrightarrow{\mathcal{V}^{*}}$-algebras

$$
\overrightarrow{P_{!}}\left(\overline{R^{*}}(E)\right) \rightarrow \overline{R^{*}}(E)
$$

By applying this to the underlying objects in $\mathcal{E}$ of $R$ we obtain a morphism of $\overrightarrow{\mathcal{V}}^{Z}$ algebras

$$
\overrightarrow{P_{!}}(|P|) \rightarrow|P| .
$$

As left adjoints preserve co-products this lifts to a morphism of co- $\mathcal{V}^{*}$-algebra objects in $\overrightarrow{V^{*}}$

$$
\overrightarrow{P !}(P) \rightarrow P
$$

From the definition of $\odot$ we can rewrite this as

$$
P \odot P \rightarrow P \text {. }
$$

That this is associative comes from its origins. This morphism started life as the 
morphism of objects in $\operatorname{Set}^{Z}$

$$
\mathcal{E}(|R|,|R|) \rightarrow \mathcal{V}^{*}\left(R^{*}(|R|), R^{*}(|R|)\right) \subseteq \operatorname{Set}^{Z}(\mathcal{E}(|R|,|R|), \mathcal{E}(|R|,|R|))
$$

which is adjoint to the morphism

$$
\mathcal{E}(|R|,|R|) \times \mathcal{E}(|R|,|R|) \rightarrow \mathcal{E}(|R|,|R|)
$$

and this is clearly associative.

For the unit, we observe that the identity morphism on $|R|$ defines a morphism of objects in $\left(\operatorname{Set}^{Z}\right)^{Z}$

$$
\operatorname{diag}(\{*\}) \rightarrow \mathcal{E}(|R|,|R|)
$$

where diag: Set $\rightarrow\left(\mathrm{Set}^{Z}\right)^{Z}$ is the diagonal functor defined on objects by

$$
\operatorname{diag}(X)=\left(z \mapsto\left(z^{\prime} \mapsto\left\{\begin{array}{ll}
X & \text { if } z=z^{\prime} \\
\emptyset & \text { otherwise }
\end{array}\right)\right)\right.
$$

and in the obvious way on morphisms.

The right hand side of (5) is the underlying object in $\left(\mathrm{Set}^{Z}\right)^{Z}$ of an object in $\mathcal{V}^{* Z}$ and so we have an adjoint $\mathcal{V}^{* Z}$-morphism

$$
\mathfrak{F}_{\mathcal{V}^{*}}^{Z} \operatorname{diag}(\{*\}) \rightarrow R^{*}(|R|) \text {. }
$$

The free $\mathcal{V}^{*}$-algebra functor on $\operatorname{diag}(\{*\})$ is (up to canonical isomorphism) the underlying $\mathcal{V}^{* Z}$-algebra of the unit, $I$, of the Tall-Wraith monoidal structure on $\mathcal{V}^{*} \mathcal{V}^{* c}$. It is straightforward to show that the morphism in (6) lifts to a morphism of co- $\mathcal{V}^{*}$-algebra objects in $\mathcal{V}^{*}$

$$
I \rightarrow R^{*}(R) .
$$

The object, $R^{*}(R)$, in $\mathcal{V}^{*} \mathcal{V}^{* c}$ is the underlying object in $\mathcal{V}^{*} \mathcal{V}^{* c}$ of the object, $\ddot{R^{*}} \mathfrak{E}(R)$, in $\dot{\mathcal{V}}^{*} \mathcal{V}^{* c}$, whence we obtain a morphism of objects in $\dot{\mathcal{V}}^{*} \mathcal{V}^{* c}$

$$
\mathfrak{D}^{c}(I) \rightarrow \ddot{R}^{*} \mathfrak{E}(R) .
$$

Applying $\mathfrak{R}$ and $\mathfrak{C}$ to this does not change the source as that is already an object in $\overrightarrow{\mathcal{V}^{*}}$. We therefore have a morphism of objects in $\overrightarrow{\mathcal{V}^{*}} \mathcal{V}^{* c}$

$$
\mathfrak{D}^{c}(I) \rightarrow{\overline{R^{*}}}^{Z}(R)=P .
$$

As this originated from the inclusion of the identity morphism on $|R|$, it is clear that this is a unit for the product defined above. We therefore have the structure of a Tall-Wraith $\overrightarrow{\mathcal{V}^{*}}$-monoid.

Returning to (4) we see that it defines a morphism in $\overrightarrow{\mathcal{V}^{*}}$

$$
P \odot\left(\overline{R^{*}}(E)\right) \rightarrow \overline{R^{*}}(E)
$$

and it is obvious that this is compatible with the monoidal structure, thus making $\overline{R^{*}}(E)$ a module for the Tall-Wraith $\overrightarrow{\mathcal{V}^{*}}$-monoid.

Applying this to a cohomology functor we obtain the following result, giving Theorems $\mathrm{A}$ and $\mathrm{B}$ of the introduction. 
Corollary 5.4. Let $E^{*}$ be a graded, multiplicative, commutative cohomology theory with representing objects $\underline{E}_{*}$. Let $\mathcal{A}_{E^{*}}^{*}$ be the variety of graded, commutative, $E^{*}$ algebras. Suppose that $E_{*}\left(\underline{E}_{k}\right)$ is free as an $E^{*}$-module for all $k$. Then $E^{*}\left(\underline{E}_{*}\right)$ is a

Tall-Wraith $\overrightarrow{\mathcal{A}_{E^{*}}^{*}}$-monoid and for a space $X, \overrightarrow{E^{*}}(X)$ is a module for this monoid.

Proof. In this case we have the following ingredients.

1. $\mathcal{E}$ is the category of spaces of the homotopy type of a $C W$-complex, which is closed under finite products.

2. $\mathcal{V}^{*}$ is $\mathcal{A}_{E^{*}}^{*}$, the variety of graded, commutative $E^{*}$-algebras, with indexing set $\mathbb{Z}$.

3. The filtration functor, $\mathfrak{E}: \mathcal{E} \rightarrow \stackrel{\leftrightarrow}{\mathcal{E}}$, is the ind-finite filtration functor defined by the subcategory of finite $C W$-complexes; see Example 1 of 3.10 for the projective version.

4. The $\mathcal{V}^{*}$-algebra object, $\underline{E}_{*}$, in $\mathcal{E}$ is provided by Brown's representability theorem.

The freeness condition on the cohomology theory in the statement of this corollary ensures that the cohomology theory satisfies the (completed) Künneth formula for its own representing spaces and thus satisfies the required "product to co-product" condition. Incidentally, this condition also ensures that the cohomology operations are already objects in $\overrightarrow{\mathcal{V}^{*}}$ and so the $\mathfrak{C}$ functor simply regards them in $\overrightarrow{\mathcal{V}^{*}}$ rather than $\overrightarrow{\mathcal{V}}^{*}$.

The filtration functor satisfies its required condition since every filtration obtained by applying the filtration functor has a final class of finite objects and it is clear that such objects have the discrete filtration.

We can therefore apply the work of this section to obtain the desired result.

\subsection{Co-operations}

Now we turn to co-operations; that is, to the Hopf ring associated to a suitable cohomology theory. In fact, we do not need to assume that the homology theory used to produce the Hopf ring is that associated to the original cohomology theory. Moreover, by using the standard categorical trick of swapping a category for its dual we can also consider the set of operations from one suitable cohomology theory to another.

In the general setting, we have the following ingredients.

1. A category $\mathcal{E}$ which is closed under finite products.

2. A complete, co-complete, extremally well-powered, (epi, extremal mono) category, $\mathcal{D}$, with the property that epimorphisms and filtered co-limits commute with finite products.

3. A graded variety of algebras, $\mathcal{V}^{*}$.

4. A filtration functor $\mathfrak{E}: \mathcal{E} \rightarrow \stackrel{\leftrightarrow}{\mathcal{E}}$.

5. A covariant functor $\mathfrak{H}: \mathcal{E} \rightarrow \mathcal{D}$.

6. A $\mathcal{V}^{*}$-algebra object, $R$, in $\mathcal{E}$.

The conditions that we require are as follows. 
1. For each finite subset $Z_{0} \subseteq Z$, the natural $\overrightarrow{\mathcal{V}^{*}}$-morphism

$$
\coprod_{z \in Z_{0}} \overline{R^{*}}(|R|(z)) \rightarrow \overline{R^{*}}\left(\prod_{z \in Z_{0}}|R|(z)\right)
$$

is an isomorphism.

2. For each finite subset $Z_{0} \subseteq Z$, the natural $\overleftarrow{\mathcal{D}}$-morphism

$$
\overline{\mathfrak{H}}\left(\prod_{z \in Z_{0}}|R|(z)\right) \rightarrow \prod_{z \in Z_{0}} \overline{\mathfrak{H}}(|R|(z))
$$

is an isomorphism; where $\overline{\mathfrak{H}}: \mathcal{E} \rightarrow \overleftarrow{\mathcal{D}}$ is the composition

$$
\mathcal{E} \stackrel{\mathfrak{E}}{\longrightarrow} \stackrel{\leftrightarrow}{\mathcal{E}} \stackrel{\leftrightarrow \mathfrak{H}}{\longrightarrow} \stackrel{\leftrightarrow}{\mathcal{D}} \stackrel{\Re}{\longrightarrow} \stackrel{\leftarrow}{\mathcal{D}} \stackrel{\mathfrak{C}}{\longrightarrow} \overleftarrow{\mathcal{D}}
$$

3. The filtration functor $\mathfrak{E}: \mathcal{E} \rightarrow \stackrel{\leftrightarrow}{\mathcal{E}}$ has the following property. The inductive filtration $\mathfrak{E}(E)$ for an object, $E$, in $\mathcal{E}$ has a final family, say $\left\{E_{\lambda}\right\}$, such that each $\mathfrak{E}\left(E_{\lambda}\right)$ is an iso-filtration.

The second condition obviously implies the following result.

Lemma 5.5. The object, $\overline{\mathfrak{H}}^{Z}(|R|)$, in $\overleftarrow{\mathcal{D}}^{Z}$ underlies a $\mathcal{V}^{*}$-algebra object in $\overleftarrow{\mathcal{D}}$

The full notation for this $\mathcal{V}^{*}$-algebra object in $\overleftarrow{\mathcal{D}}$ would be $\overline{\mathfrak{H}}^{Z}(R)$. We shall shorten this to $H$.

We therefore have a contravariant functor $H^{*}: \overleftarrow{\mathcal{D}} \rightarrow \mathcal{V}^{*}$. Using the standard categorical trick, we apply the first construction of Definition 3.30 to this contravariant functor to obtain a lift

$$
\overrightarrow{H^{*}}: \overleftarrow{\mathcal{D}} \rightarrow \overrightarrow{\mathcal{V}^{*}}
$$

Recall that $P={\overrightarrow{R^{*}}}^{Z}(R)$ is, by Corollary 5.3, the underlying object in $\overrightarrow{\mathcal{V}^{*}} \mathcal{V}^{* c}$ of a Tall-Wraith $\overrightarrow{\mathcal{V}^{*}}$-monoid. We have the following result by a similar argument to that in the previous section.

Proposition 5.6. The $\mathcal{V}^{*}$-algebra object, $H$, in $\overleftarrow{\mathcal{D}}$ is a module for the Tall-Wraith $\overrightarrow{\mathcal{V}^{*}}$-monoid, $P$. For an object, E, in $\mathcal{E}$, the $\overrightarrow{\mathcal{V}^{*}}$-algebra

$$
\overrightarrow{H^{*}}(\overline{\mathfrak{H}}(E))
$$

is a module for $P$ and the morphism of $\overrightarrow{\mathcal{V}^{*}}$-algebras

$$
\overrightarrow{R^{*}}(E) \rightarrow \overrightarrow{H^{*}}(\overline{\mathfrak{H}}(E))
$$

induced by the morphism of objects in $\operatorname{Set}^{Z}$

$$
\mathcal{E}(E,|R|) \rightarrow \overleftarrow{\mathcal{D}}\left(\overline{\mathfrak{H}}(E), \overline{\mathfrak{H}}^{Z}(|R|)\right)=\overleftarrow{\mathcal{D}}(\overline{\mathfrak{H}}(E),|H|)
$$

is a morphism of P-modules. 
Notice that we use the variance switch to turn the more obvious co-action morphism

$$
H \rightarrow H \odot P
$$

into an action morphism

$$
P \circledast H \rightarrow H .
$$

By replacing $\mathcal{D}$ by $\mathcal{D}^{\text {op }}$ we can consider contravariant functors $\mathfrak{G}: \mathcal{E} \rightarrow \mathcal{D}$. The above conditions on $\mathcal{D}$ are now conditions on $\mathcal{D}^{\text {op }}$ and so need to be replaced by their duals to get the required conditions on $\mathcal{D}$. With the obvious notation we have the following result.

Proposition 5.7. The co- $\mathcal{V}^{*}$-algebra object, $G=\overline{\mathfrak{G}}^{Z}(R)$, in $\overrightarrow{\mathcal{D}}$ is a module for the Tall-Wraith $\overrightarrow{\mathcal{V}^{*}}$-monoid, $P$. For an object, E, in $\mathcal{E}$, the $\overrightarrow{\mathcal{V}^{*}}$-algebra

$$
\overrightarrow{G_{*}}(\overline{\mathfrak{G}}(E))
$$

is a module for $P$ and the morphism of $\overrightarrow{\mathcal{V}^{*}}$-algebras

$$
\overline{R^{*}}(E) \rightarrow \overrightarrow{G_{*}}(\overline{\mathfrak{G}}(E))
$$

induced by the morphism of objects in $\operatorname{Set}^{Z}$

$$
\mathcal{E}(E,|R|) \rightarrow \overrightarrow{\mathcal{D}}\left(\overline{\mathfrak{G}}^{Z}(|R|), \overline{\mathfrak{G}}(E)\right)=\overrightarrow{\mathcal{D}}(|G|, \overline{\mathfrak{G}}(E))
$$

is a morphism of P-modules.

We now obtain Theorem $\mathrm{C}$ of the introduction by applying the above results to suitable homology and cohomology functors.

Firstly, applying Proposition 5.6 to an appropriate homology functor we obtain the following description of an enriched Hopf ring.

Proposition 5.8. Let $E^{*}$ and $F^{*}$ be graded, multiplicative, commutative cohomology theories with representing objects $\underline{E}_{*}$ and $\underline{F}_{*}$ respectively. Let $\mathcal{A}_{E^{*}}^{*}$ be the variety of graded, commutative, $E^{*}$-algebras.

Suppose that $F_{*}\left(\underline{E}_{k}\right)$ is free as an $F^{*}$-module for all $k$ and that $E_{*}\left(\underline{E}_{k}\right)$ is free as an $E^{*}$-module for all $k$. Then $F_{*}\left(\underline{E}_{*}\right)$ is a module for the Tall-Wraith $\overrightarrow{\mathcal{A}_{E^{*}}^{*}}$-monoid $E^{*}\left(\underline{E}_{*}\right)$

For a space $X$ with the property that $F_{*}(X)$ is free as an $F^{*}$-module, the obvious morphism

$$
E^{*}(X) \rightarrow \overrightarrow{\left(\bar{F}_{*}^{Z}\left(\underline{E}_{*}\right)\right)^{*}}\left(F_{*}(X)\right)
$$

is a morphism of $E^{*}\left(\underline{E}_{*}\right)$-modules.

In the last part of this proposition our intermediate category is that of $F^{*}$-coalgebras. The conditions on $E^{*}$ and $F^{*}$ ensure that each $F_{*}\left(\underline{E}_{k}\right)$ is an $F^{*}$-co-algebra and thus that $F_{*}\left(E_{*}\right)$ is an $E^{*}$-algebra object in $F^{*}$-co-algebras. The condition on the space $X$ also ensures that $F_{*}(X)$ is an $F^{*}$-co-algebra. By combining results from the literature, namely [Bar74, Fox93, Por06], it can be shown that the category 
of $F^{*}$-co-algebras does have all the categorical properties necessary for our results to apply.

Secondly, applying Proposition 5.7 to an appropriate cohomology functor we obtain the following description of the structure of the operations from one cohomology theory to another.

Proposition 5.9. Let $E^{*}$ and $F^{*}$ be graded, multiplicative, commutative cohomology theories with representing objects $\underline{E}_{*}$ and $\underline{F}_{*}$ respectively. Let $\mathcal{A}_{E^{*}}^{*}$ be the variety of graded, commutative, $E^{*}$-algebras.

Suppose that $F_{*}\left(\underline{E}_{k}\right)$ is free as an $F^{*}$-module for all $k$ and that $E_{*}\left(\underline{E}_{k}\right)$ is free as an $E^{*}$-module for all $k$. Then $F^{*}\left(\underline{E}_{*}\right)$ is a module for the Tall-Wraith $\overrightarrow{\mathcal{A}_{E^{*}}^{*}}$-monoid $E^{*}\left(\underline{E}_{*}\right)$.

For a space $X$, the obvious morphism

$$
E^{*}(X) \rightarrow \overrightarrow{\left(\vec{F}^{*}\left(\underline{E}_{*}\right)\right)_{*}}\left(F^{*}(X)\right)
$$

is a morphism of $E^{*}\left(\underline{E}_{*}\right)$-modules.

As is well-known, the assumptions in Proposition 5.8 are sufficient to ensure that the object $F_{*}\left(\underline{E}_{*}\right)$ is a Hopf ring; that is, an $E^{*}$-algebra object in the category of $F^{*}$-co-algebras; see [RW77] and [Wil00]. In [BJW95] the authors expanded this to the notion of an enriched Hopf ring. The enriched part corresponds to the action of the Tall-Wraith monoid $E^{*}\left(\underline{E}_{*}\right)$.

It is not usual to view homology as topologised. Indeed, the inductive filtration does not really give these structures a topology. Even though there is a notion of an inductive topology, this is not how one should regard the inductive filtration on the homology groups of a space. Rather, it is a dual topology in that it is the structure required to induce a topology on the dual, or more generally on the result of applying some other contravariant functor. Thus we have not altered the usual view of homology as being discrete.

This concept of an inductive filtration was not used explicitly in [Boa95] and [BJW95]. On the other hand, it is there under the surface. In our language, what the authors of [Boa95] and [BJW95] do is to define an inductive filtration functor on the category of modules over some ring. They then show that this is compatible with taking the usual filtration functor on the category of topological spaces and so rather than having to lift the homology functor to the filtered category they first apply the (unfiltered) homology functor and follow it by the filtration functor on the category of modules.

The Milnor short exact sequence for homology shows that the homology of a space is always iso-filtered and so the issue of passing to the "completion" does not arise in homology.

As noted above, the category of $F^{*}$-co-algebras has all the properties necessary for our results to apply. This means that one can "do" general algebra in this category and thus we can apply the results of Section 2. From Proposition 2.19 and Theorem 2.24 we deduce the existence of free $\mathcal{V}^{*}$-algebra objects for any variety of graded algebras. In particular, if $\mathcal{V}^{*}$ is the category of commutative $E^{*}$-algebras we recover the result of Ravenel and Wilson, [RW77, $§ 1]$, that free Hopf rings exist. If $\mathcal{V}^{*}$ is the category 
of $E^{*}$-modules we recover the result of Hunton and Turner, [HT98, §2], that free co-algebraic modules exist.

In future work we expect to give descriptions of the Tall-Wraith monoids of unstable operations for various cohomology theories, including the Morava $K$-theories.

\section{References}

[Bar74] Michael Barr, Coalgebras over a commutative ring, J. Algebra 32(3) (1974), 600-610.

[Ber98] George M. Bergman, An Invitation to General Algebra and Universal Constructions, Henry Helson, Berkeley, CA, 1998.

[BH96] George M. Bergman and Adam O. Hausknecht, Co-groups and co-rings in categories of associative rings, vol. 45, Mathematical Surveys and Monographs, American Mathematical Society, Providence, RI, 1996.

[BJW95] J. Michael Boardman, David Copeland Johnson and W. Stephen Wilson, Unstable operations in generalized cohomology, Handbook of Algebraic Topology 8, North-Holland, Amsterdam, 1995, pp. 687-682.

[Boa95] J. Michael Boardman, Stable operations in generalized cohomology, Handbook of Algebraic Topology, North-Holland, Amsterdam, 1995, pp. 585-686.

[BW05] James Borger and Ben Wieland, Plethystic algebra, Adv. Math. 194(2) (2005), 246-283.

[CMS02] Dena S. Cowen Morton and Neil Strickland, The Hopf rings for $K O$ and KU, J. Pure Appl. Algebra 166(3) (2002), 247-265.

[Fox93] Thomas F. Fox, The construction of cofree coalgebras, J. Pure Appl. Algebra 84(2) (1993), 191-198.

[Fre66] P. Freyd, Algebra valued functors in general and tensor products in particular, Colloq. Math. 14 (1966), 89-106.

[HS73] Horst Herrlich and George E. Strecker, Category Theory: An Introduction, Allyn and Bacon Series in Advanced Mathematics, Allyn and Bacon Inc., Boston, Mass., 1973.

[HT98] John R. Hunton and Paul R. Turner, Coalgebraic algebra, J. Pure Appl. Algebra 129(3) (1998), 297-313.

[KPMS82] W. Kühnel, M. Pfender, J. Meseguer and I. Sols, Algebras with actions and automata, Internat. J. Math. Math. Sci. 5(1) (1982), 61-85.

[Por06] Hans-E. Porst, On corings and comodules, Arch. Math. (Brno) 42(4) (2006), 419-425.

[RW77] Douglas C. Ravenel and W. Stephen Wilson, The Hopf ring for complex cobordism, J. Pure Appl. Algebra 9(3) (1976/77), 241-280.

[RW96] Douglas C. Ravenel and W. Stephen Wilson, The Hopf ring for $P(n)$, Canad. J. Math. 48(5) (1996), 1044-1063. 
[SW08] Andrew Stacey and Sarah Whitehouse, Stable and unstable operations in mod $p$ cohomology theories, Algebr. Geom. Topol. 8(2) (2008), 10591091.

[SW09] Andrew Stacey and Sarah Whitehouse, Tall-Wraith monoids, in preparation, 2009.

[TW70] D. O. Tall and G. C. Wraith, Representable functors and operations on rings, Proc. London Math. Soc. (3) 20 (1970), 619-643.

[Wil84] W. Stephen Wilson, The Hopf ring for Morava K-theory, Publ. Res. Inst. Math. Sci. 20(5) (1984), 1025-1036.

[Wil00] W. Stephen Wilson, Hopf rings in algebraic topology, Expo. Math. 18(5) (2000), 369-388.

Andrew Stacey stacey@math.ntnu.no

http://www.math.ntnu.no/ stacey

Institutt for Matematiske Fag, NTNU, Trondheim, Norway

Sarah Whitehouse S.Whitehouse@sheffield.ac.uk

http://www.sarah-whitehouse.shef.ac.uk

Department of Pure Mathematics, University of Sheffield, Sheffield, UK 